\title{
DYNAMIC TAGS FOR SECURITY PROTOCOLS
}

\author{
MYRTO ARAPINIS $^{a}$, STÉPHANIE DELAUNE $^{b}$, AND STEVE KREMER $^{c}$ \\ ${ }^{a}$ School of Informatics, University of Edinburgh, UK \\ e-mail address: marapini@inf.ed.ac.uk \\ ${ }^{b}$ LSV, CNRS \& ENS Cachan, France \\ e-mail address: delaune@lsv.ens-cachan.fr \\ ${ }^{c}$ Inria Nancy - Grand Est \& LORIA, France \\ e-mail address: Steve.Kremer@inria.fr
}

\begin{abstract}
The design and verification of cryptographic protocols is a notoriously difficult task, even in symbolic models which take an abstract view of cryptography. This is mainly due to the fact that protocols may interact with an arbitrary attacker which yields a verification problem that has several sources of unboundedness (size of messages, number of sessions, etc.).

In this paper, we characterize a class of protocols for which deciding security for an unbounded number of sessions is decidable. More precisely, we present a simple transformation which maps a protocol that is secure for a bounded number of protocol sessions (a decidable problem) to a protocol that is secure for an unbounded number of sessions. The precise number of sessions that need to be considered is a function of the security property and we show that for several classical security properties a single session is sufficient. Therefore, in many cases our results yields a design strategy for security protocols: (i) design a protocol intended to be secure for a single session; and (ii) apply our transformation to obtain a protocol which is secure for an unbounded number of sessions.
\end{abstract}

\section{INTRODUCTION}

Security protocols are distributed programs which aim at guaranteeing properties such as confidentiality of data, authentication of participants, etc. The security of these protocols relies on the one hand on the security of cryptographic primitives, e.g. encryption and digital signatures, and on the other hand on the concurrency-related aspects of the protocols themselves. History has shown that even if cryptography is supposed to be perfect, such as in the classical Dolev-Yao model [20, the correct design of security protocols is notoriously error-prone. See for instance [13] for an early survey on attacks. These difficulties come mainly from two sources of unboundedness: a protocol may be executed several times (we need to consider several protocol sessions) and the attacker is allowed to build messages of unbounded size. Indeed, secrecy is known to be undecidable when an unbounded number

2012 ACM CCS: [Security and privacy]: Formal methods and theory of security - Formal security models.

Key words and phrases: formal methods, security protocols, verification.

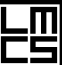

DOI:10.2168/LMCS-10(2:11)2014
(C) M. Arapinis, S. Delaune, and S. Kremer

(C) Creative Commons 
of sessions is allowed, even if the message size is bounded [21]. However, when the number of sessions is bounded, and even without assuming a bounded message size, the problem becomes co-NP-complete [30]. Moreover, special purpose verification tools (e.g. 4]) exist which are highly efficient when the number of sessions is small.

In this paper we propose a protocol transformation which maps a protocol that is secure for a bounded number of sessions to a protocol that is secure for an unbounded number of sessions. The exact number of sessions that need to be considered depends on the security property under study. We express security properties in a temporal logic with past similar to the logics of [16, 17]. This logic is expressive enough to model security properties such as secrecy and several flavors of non-injective authentication properties. As we will see for these classical security properties verifying a single session will be sufficient and our result provides a strategy to design secure protocols: (i) design a protocol intended to be secure for a single session; and (ii) apply our transformation and obtain a protocol which is secure for an unbounded number of sessions.

Our transformation. Suppose that $\Pi$ is a protocol between $k$ participants $A_{1}, \ldots, A_{k}$. Our transformation adds to $\Pi$ a preamble in which each participant sends a freshly generated nonce $N_{i}$ together with his identity to all other participants. This allows each participant to compute a dynamic, session-dependent $\operatorname{tag}\left\langle A_{1}, N_{1}\right\rangle, \ldots,\left\langle A_{k}, N_{k}\right\rangle$ that will be used to tag each encryption and signature in $\Pi$. Our transformation is surprisingly simple and does not require any cryptographic protection of the preamble, i.e., an active attacker is allowed to interfere with this preliminary phase. Intuitively, the security relies on the fact that the participant $A_{i}$ decides on a given tag for a given session which is ensured to be fresh as it contains his own freshly generated nonce $N_{i}$. The transformation is computationally light as it does not add any cryptographic application; it may merely increase the size of messages to be encrypted or signed. The transformation applies to a large class of protocols, which may use symmetric and asymmetric encryption, digital signature and hash functions.

We may note that, en passant, we identify a class of tagged protocols for which security is decidable for an unbounded number of sessions. This directly follows from our main result as it stipulates that verifying security for a bounded number of protocol sessions is sufficient to conclude security for an unbounded number of sessions.

Related Work. The kind of compiler we propose here has also been investigated in the area of cryptographic design in computational models, especially for the design of group key exchange protocols. For example, Katz and Yung [23] proposed a compiler which transforms a key exchange protocol secure against a passive eavesdropper into an authenticated protocol which is secure against an active attacker. Earlier work includes compilers for 2-party protocols (e.g. [7]). In the symbolic model, recent works [18, 6] allow one to transform a protocol which is secure in a weak sense (roughly no attacker [18] or just a passive one [6] and a single session) into a protocol secure in the presence of an active attacker and for an unbounded number of sessions. All of these prior works share however a common drawback: the proposed transformations make heavy use of cryptography. This is mainly due to the fact that the security assumptions made on the input protocol are rather weak. As already mentioned in [18], it is important, from an efficiency perspective to lighten the use of cryptographic primitives. In this work, we succeed in doing so at the price of requiring stronger security guarantees on the input protocol. However, we argue that this is acceptable since efficient automatic tools exist to decide this security criterion on the 
input protocols. Recently, our transformation has also been adapted to the case of offline guessing attacks in password-based protocols [11]. On the one hand the result presented in [11] is more complicated as it considers a more complex security property but, on the other hand, the proof is simplified by the fact that the password is the only secret shared between different sessions.

We can also compare our work with existing decidable protocol classes for an unbounded number of sessions. An early result is the PTIME complexity result by Dolev et al. [19] for a restricted class, called ping-pong protocols. Other classes have been proposed by Ramanujam and Suresh [28, 29], and Lowe [26]. However, in both cases, temporary secrets, composed keys and ciphertext forwarding are not allowed which discards protocols (even their tagged version), such as the Yahalom protocol [13].

Different kinds of tags have also been considered in [12, 3, 17, 9, 28. However these tags are static and have a different aim. While our dynamic tagging scheme avoids confusing messages from different sessions, these static tags avoid confusing different messages inside the same session and do not prevent that the same message is reused in two different sessions. Under some additional assumptions (e.g. no temporary secret, no ciphertext forwarding), several decidability results [29, 26] have been obtained by showing that it is sufficient to consider one session per role. But those results cannot deal with protocols which rely on ciphertext forwarding and/or temporary secrets. In the framework we consider here, the question whether such static tags would be sufficient to obtain decidability is still an open question (see [3]). In a similar way, static tags have also been used by Heather et al. 222] to avoid type confusion attacks.

Finally, we may note that our tags are reminiscent of session tags in the UC framework [10] and in particular the method proposed by Barak et al. [5] for computing them. However, in addition to the important differences in the models, these works do not propose a general, systematic transformation which guarantees (joint state) composition between sessions.

This paper can be seen as an extended and enriched version of [2]. In [2], our reduction result was only established for the secrecy property whereas we consider here a larger class of security properties that includes several levels of authentication. Moreover, the proof of our main result is now self-contained and does not rely anymore on the constraint solving procedure presented in [15].

Outline of the paper. Our paper is organized in two parts: Part I presents our result and all the necessary background for the result to be formally stated and Part II is devoted to giving an overview of the proof of the result (for readability some of the more technical proofs are only given in an appendix).

In Part I we first introduce our abstract representation of protocol messages (Section 2) and our formal models for security protocols (Section 3) and properties (Section 4). Next, in Section 5. we formally define our protocol transformation and state our main result which guarantees that attacks only require a bounded number of sessions.

In Part II we give an overview of our proof. In Section 6 we define a transformation on protocol executions and show that a transformed execution

(i) has several good properties (it is both valid and well-formed), and

(ii) preserves the satisfaction of attack formulas. 
In Section 7 we show that we can restrict the sessions that are involved in a valid, wellformed execution while preserving

(i) validity and well-formedness, and

(ii) satisfaction of attack formulas.

Finally, in Section 8, we use the results from the previous two sections to prove our main result.

\section{— PART I: Presentation of our reduction result -}

\section{Messages And intruder Capabilities}

2.1. Messages. We use an abstract term algebra to model the messages of a protocol. For this we fix several disjoint sets. We consider an infinite set of agents $\mathcal{A}=\{\epsilon, a, b \ldots\}$ with the special agent $\epsilon$ standing for the attacker and an infinite set of agent variables $\mathcal{X}=\left\{x_{A}, x_{B}, \ldots\right\}$. We also need to consider an infinite set of names $\mathcal{N}=\{n, m \ldots\}$ and an infinite set of variables $\mathcal{Y}=\{y, z, \ldots\}$. Among this set of names, we consider the infinite set of names $\mathcal{N}_{\epsilon}=\left\{n^{\epsilon}, \ldots\right\}$ that corresponds to names known initially by the attacker. We consider the following signature $\mathcal{F}=\{$ encs $/ 2$, enca $/ 2$, sign $/ 2,\langle\rangle / 2, \mathrm{~h} / 1$, pub $/ 1$, priv $/ 1$, shk $/ 2\}$. These function symbols model cryptographic primitives. The symbol \langle\rangle represents pairing. The term encs $(m, k)$ (resp. enca $(m, k))$ represents the message $m$ encrypted with the symmetric (resp. asymmetric) key $k$ whereas the term $\operatorname{sign}(m, k)$ represents the message $m$ signed by the key $k$. The function $\mathrm{h}$ models a hash function whereas $\operatorname{pub}(a)$ and $\operatorname{priv}(a)$ are used to model the public and the private key respectively of an agent $a$, and $\operatorname{shk}(a, b)(=$ $\operatorname{shk}(b, a))$ is used to model the long-term symmetric key shared by agents $a$ and $b$. Names are used to model atomic data such as nonces. The set of terms is defined inductively by the following grammar:

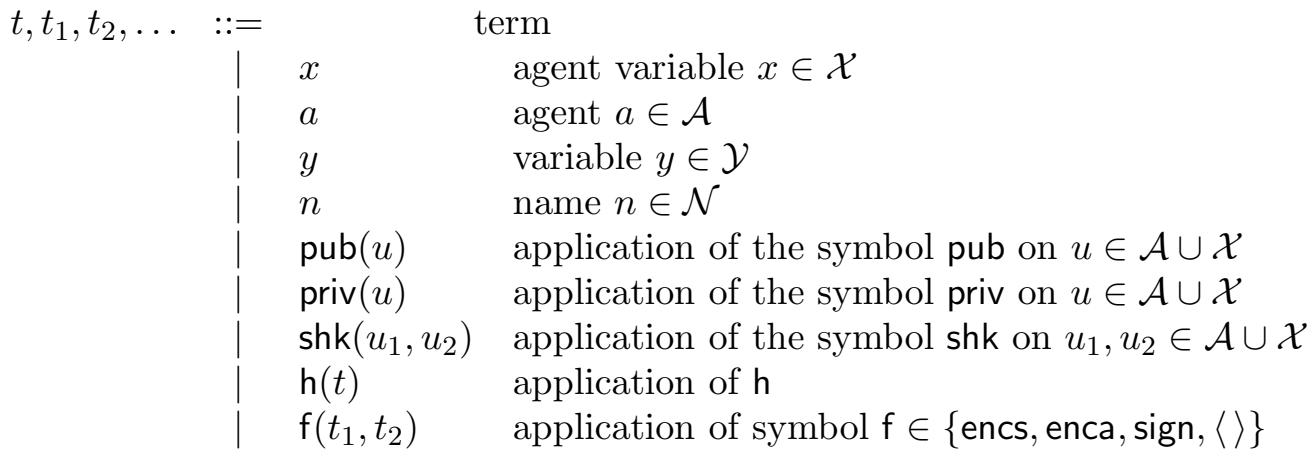

We sometimes write $\left\langle t_{1}, \ldots, t_{n}\right\rangle$ instead of writing $\left\langle t_{1},\left\langle\ldots,\left\langle t_{n-1}, t_{n}\right\rangle \ldots\right\rangle\right\rangle$. We say that a term is ground if it has no variable. We consider the usual notations for manipulating terms. A position $p$ in a term $t$ is a sequence of integers. The empty sequence $\varepsilon$ denotes the top-most position. The subterm of $t$ at position $p$ is written $\left.t\right|_{p}$. We write vars $(t)$ (resp. names $(t)$, agents $(t)$ ) for the set of variables (resp. names, agents) occurring in $t$. We write $\mathrm{St}(t)$ for the set of syntactic subterms of a term $t$ and define the set of cryptographic subterms of a term $t$ as $\operatorname{CryptSt}(t)=\left\{\mathrm{f}\left(t_{1}, \ldots, t_{n}\right) \in \mathrm{St}(t) \mid \mathrm{f} \in\{\right.$ encs, enca, sign, $\left.\mathrm{h}\}\right\}$. Moreover, we 


$$
\begin{gathered}
\frac{u}{\langle u, v\rangle} \frac{u}{\operatorname{encs}(u, v)} \quad \frac{u}{\operatorname{enca}(u, v)} \frac{u}{\operatorname{sign}(u, v)} \frac{v}{\mathrm{~h}(u)} \\
\frac{\langle u, v\rangle}{u} \quad \frac{\langle u, v\rangle}{v} \quad \frac{\operatorname{encs}(u, v) v}{u} \quad \frac{\operatorname{enca}(u, \operatorname{pub}(v))}{u} \operatorname{priv(v)}
\end{gathered}
$$

Figure 1: Intruder deduction system.

define the set of long-term keys as lgKeys $=\{\operatorname{priv}(a) \mid a \in \mathcal{A}\} \cup\{\operatorname{shk}(a, b) \mid a, b \in \mathcal{A}\}$ and the set of long-term keys of a term $t$ as

$$
\operatorname{lgKeys}(t)=\{\operatorname{priv}(u) \mid \operatorname{pub}(u) \in \mathrm{St}(t) \text { or } \operatorname{priv}(u) \in \operatorname{St}(t)\} \cup\left\{\operatorname{shk}\left(u_{1}, u_{2}\right) \in \operatorname{St}(t)\right\} .
$$

and we define $\mathcal{K}_{\epsilon}=\{\operatorname{priv}(\epsilon)\} \cup\{\operatorname{shk}(a, \epsilon) \mid a \in \mathcal{A}\}$. Intuitively $\mathcal{K}_{\epsilon}$ represents the set of long-term keys of the attacker. An atom is a long-term key, a name or a variable.

We define the set of plaintexts of a term $t$ as the set of atoms that occur in plaintext position, i.e.

- plaintext $(\mathrm{h}(u))=\operatorname{plaintext}(\mathrm{f}(u, v))=\operatorname{plaintext}(u)$ for $\mathrm{f} \in\{$ encs, enca, sign $\}$,

- plaintext $(\langle u, v\rangle)=\operatorname{plaintext}(u) \cup \operatorname{plaintext}(v)$, and

- $\operatorname{plaintext}(u)=\{u\} \quad$ otherwise.

All these notions are extended to sets of terms and to other kinds of term containers as expected. We denote by $\# S$ the cardinality of a set $S$. Substitutions are written $\sigma=\left\{x_{1} \mapsto t_{1}, \ldots, x_{n} \mapsto t_{n}\right\}$ where its domain is $\operatorname{dom}(\sigma)=\left\{x_{1}, \ldots, x_{n}\right\}$. The substitution $\sigma$ is ground if all the $t_{i}$ are ground. The application of a substitution $\sigma$ to a term $t$ is written $\sigma(t)$ or $t \sigma$. Two terms $t_{1}$ and $t_{2}$ are unifiable if $t_{1} \sigma=t_{2} \sigma$ for some substitution $\sigma$, that is called a unifier. We denote by mgu $\left(t_{1}, t_{2}\right)$ the most general unifier of $t_{1}$ and $t_{2}$.

Example 2.1. Let $t=\operatorname{encs}(\langle n, a\rangle, \operatorname{shk}(a, b))$. We have that $\operatorname{vars}(t)=\emptyset$, i.e. $t$ is ground, names $(t)=\{n\}, \operatorname{agents}(t)=\{a, b\}, \operatorname{lgKeys}(t)=\{\operatorname{shk}(a, b)\}$, plaintext $(t)=\{n, a\}$, and $\operatorname{St}(t)=\{t,\langle n, a\rangle, \operatorname{shk}(a, b), n, a\}$. The terms shk $(a, b), a, n$ and $\operatorname{priv}(a)$ are atoms.

2.2. Intruder capabilities. We model the intruder's abilities to construct new messages by the deduction system given in Figure 1. The first line describes the composition rules. The second line describes the decomposition rules. The intuitive meaning of these rules is that an intruder can compose new messages by pairing, encrypting, signing and hashing previously known messages provided he has the corresponding keys. Conversely, he can decompose messages by projecting or decrypting provided he has the decryption keys. Our optional rule expresses that an intruder can retrieve the whole message from its signature. Whether this property holds depends on the actual signature scheme. Therefore we consider this rule to be optional. Our results hold in both cases.

Definition 2.2 (deducible). We say that a term $u$ is deducible from a set of terms $T$, denoted $T \vdash u$, if there exists a tree such that its root is labeled by $u$, its leaves are labeled with $v \in T \cup \mathcal{A} \cup \mathcal{N}_{\epsilon} \cup \mathcal{K}_{\epsilon} \cup\{\operatorname{pub}(a) \mid a \in \mathcal{A}\}$ and for every node labeled by $v$ having $n$ sons labeled by $v_{1}, \ldots, v_{n}$ we have that $\frac{v_{1} \ldots v_{n}}{v}$ is an instance of one of the inference rules given in Figure 1.

Example 2.3. The term $\langle n, \operatorname{shk}(a, b)\rangle$ is deducible from $\{\operatorname{encs}(n, \operatorname{shk}(a, b)), \operatorname{shk}(a, b)\}$. 
We are now able to state the following lemma that can be easily proved by induction on the proof tree witnessing $T \vdash t$.

Lemma 2.4. Let $T$ be a set of terms and $t$ be a term such that $T \vdash t$. We have that:

$$
\text { plaintext }(t) \subseteq \operatorname{plaintext}(T) \cup \mathcal{A} \cup \mathcal{N}_{\epsilon} \cup \mathcal{K}_{\epsilon} \cup\{\operatorname{pub}(a) \mid a \in \mathcal{A}\} .
$$

\section{MOdEL FOR SECURITY PROTOCOLS}

In this section, we give a language for specifying protocols and define their execution in the presence of an active attacker. Our model is similar to existing ones (see e.g. [30, 17]).

3.1. Syntax. We consider protocols specified in a language allowing parties to exchange messages built from identities and randomly generated nonces using pairing, public key, symmetric encryption, hashing and digital signatures. The individual behavior of each protocol participant is defined by a role describing a sequence of events. The main events we consider are communication events (i.e. message receptions and message transmissions) and status events to mark different stages reached by the protocol. These status events will help us specify a large class of security properties (a logic of properties is given in Section 4). These are issued by participants to denote their current state in the execution of a protocol role.

Definition 3.1 (event). An event is either

- a communication event, i.e. a message reception, denoted by $\operatorname{rcv}(m)$ or a message transmission, denoted by $\operatorname{snd}(m)$, where $m$ is a term; or

- a status event of the form $\mathrm{P}\left(t_{1}, \ldots, t_{n}\right)$ where each $t_{i}$ is a term (not necessarily ground) and $\mathrm{P} \in \mathcal{P}$ is a predicate symbol of arity $n$.

Typically, status events give information about the state of the principal. For instance, we will consider a status event that indicates that the principal has started or finished a session. The set of variables of an event is defined as expected, considering all the terms occurring in the event's specification.

Definition 3.2 (roles). A role is of the form $\lambda x_{1} \ldots \lambda x_{k} . \nu y_{1} \ldots \nu y_{p}$. seq, where:

- $X=\left\{x_{1}, \ldots, x_{k}\right\}$ is a set of agent variables, i.e. the parameters of the role corresponding to the $k$ participants of the protocol,

- $Y=\left\{y_{1}, \ldots, y_{p}\right\}$ is a set of variables: the nonces generated by the role,

- $\operatorname{seq}=\mathrm{e}_{1} ; \mathrm{e}_{2} ; \ldots ; \mathrm{e}_{\ell}$ is a sequence of events such that $(\operatorname{vars}($ seq $) \backslash X) \subseteq \mathcal{Y}$, i.e. all agent variables are parameters.

Moreover, we have that:

(1) seq satisfies the origination property, that is for any send or status event $\mathrm{e}_{i}$, for any variable $x \in \operatorname{vars}\left(\mathrm{e}_{i}\right) \backslash(X \cup Y)$, we have that $x \in \operatorname{vars}\left(\mathrm{e}_{j}\right)$ for some receive event $\mathrm{e}_{j}$ where $j<i$; and

(2) seq satisfies the plaintext origination property, that is for any send or status event $\mathrm{e}_{i}$, for any variable $x \in \operatorname{plaintext}\left(\mathrm{e}_{i}\right) \backslash(X \cup Y)$, we have that $x \in \operatorname{plaintext}\left(\mathrm{e}_{j}\right)$ for some receive event $\mathrm{e}_{j}$ where $j<i$.

The set of roles is denoted by Roles. The length of a role is the number of elements in its sequence of events. A $k$-party protocol is a mapping $\Pi:[k] \rightarrow$ Roles, where $[k]=\{1,2, \ldots, k\}$. 
The condition (11) above ensures that each variable which appears in a send or status event is a nonce, a parameter, or a variable that has been introduced in a previously received message. Condition (2) ensures that a key used for encrypting or signing cannot be extracted and used as plaintext, e.g. forbidding a sequence $\operatorname{rcv}(\operatorname{encs}(y, z)) ; \operatorname{snd}(z)$.

Example 3.3. We illustrate our protocol syntax on the familiar Needham-Schroeder publickey protocol [27]. In our syntax this protocol is modeled as follows.

$$
\begin{aligned}
\Pi(1)= & \lambda x_{A} \cdot \lambda x_{B} \cdot \nu y . & \Pi(2)= & \lambda x_{A} \cdot \lambda x_{B} \cdot \nu y^{\prime} . \\
& \operatorname{snd}\left(\operatorname{enca}\left(\left\langle y, x_{A}\right\rangle, \operatorname{pub}\left(x_{B}\right)\right)\right) ; & & \operatorname{rcv}\left(\operatorname{enca}\left(\left\langle z^{\prime}, x_{A}\right\rangle, \operatorname{pub}\left(x_{B}\right)\right)\right) ; \\
& \operatorname{rcv}\left(\operatorname{enca}\left(\langle y, z\rangle, \operatorname{pub}\left(x_{A}\right)\right)\right) ; & & \operatorname{snd}\left(\operatorname{enca}\left(\left\langle z^{\prime}, y^{\prime}\right\rangle, \operatorname{pub}\left(x_{A}\right)\right)\right) ; \\
& \operatorname{snd}\left(\operatorname{enca}\left(z, \operatorname{pub}\left(x_{B}\right)\right)\right) & & \operatorname{rcv}\left(\operatorname{enca}\left(y^{\prime}, \operatorname{pub}\left(x_{B}\right)\right)\right)
\end{aligned}
$$

The initiator, role $\Pi(1)$ played by $x_{A}$, sends to the responder, role $\Pi(2)$ played by $x_{B}$, his identity together with a freshly generated nonce $y$, encrypted with the responder's public key. The responder replies by copying the initiator's nonce and adds a fresh nonce $y^{\prime}$, encrypted by the initiator's public key. The initiator acknowledges by forwarding the responder's nonce encrypted by his public key.

Clearly, not all protocols written using the syntax above are meaningful. In particular, some of them might not be executable. For instance, a $k$-party protocol where $\Pi(1):=$ $\operatorname{rcv}(\mathrm{h}(x)) ; \operatorname{snd}(x)$ is not executable since an agent is not able to extract the content of a hash. A precise definition of executability is not relevant for our result. We only need to consider the weaker plaintext origination hypothesis (Condition 2 stated in Definition 3.2). In particular, our result also holds for non-executable protocols such as the one given above.

3.2. Semantics. In our model, a session corresponds to the instantiation of one role. This means in particular that one "normal execution" of a $k$-party protocol requires $k$ sessions, one per role1. We may want to consider several sessions corresponding to different instantiations of a same role. Since the adversary may block, redirect and send new messages, all the sessions might be interleaved in many ways. Such an interleaving is captured by the notion of a scenario.

Definition 3.4 (scenario). A scenario for a protocol $\Pi:[k] \rightarrow$ Roles is a sequence $\mathrm{sc}=$ $\left(r_{1}, \operatorname{sid}_{1}\right) \cdots\left(r_{n}, \operatorname{sid}_{n}\right)$ where $r_{i}$ is a role and sid $_{i}$ a session identifier such that $1 \leq r_{i} \leq k$, $\operatorname{sid}_{i} \in \mathbb{N} \backslash\{0\}$, the number of identical occurrences of a pair $(r$, sid $)$ is smaller than the length of the role $r$, and sid $_{i}=\operatorname{sid}_{j}$ implies $r_{i}=r_{j}$.

The condition on identical occurrences ensures that a role cannot execute more events than it contains. The last condition ensures that a session number is not reused by other roles. We say that $(r, s) \in \mathrm{sc}$ if $(r, s)$ is an element of the sequence sc.

Given a scenario and an instantiation for the parameters, we define a symbolic trace, that is a sequence of events that corresponds to the interleaving of the scenario, for which the parameters have been instantiated, fresh nonces are generated and variables are renamed to avoid name collisions between different sessions.

Definition 3.5 (symbolic trace). Let $\Pi$ be a $k$-party protocol with

$$
\Pi(j)=\lambda x_{1}^{j} \ldots \lambda x_{k}^{j} . \nu y_{1}^{j} \ldots \nu y_{p_{j}}^{j} \cdot \mathrm{e}_{1}^{j} ; \ldots ; \mathrm{e}_{\ell_{j}}^{j} \quad \text { for } 1 \leq j \leq k .
$$

\footnotetext{
${ }^{1}$ In the literature, the word session is often used in an abusive way to represent an execution of the protocol, i.e. one session per role, whereas we use it for the execution of a role.
} 
Given a scenario $\mathrm{sc}=\left(r_{1}, \operatorname{sid}_{1}\right) \cdots\left(r_{n}\right.$, sid $\left._{n}\right)$ and a function $\alpha: \mathbb{N} \rightarrow \mathcal{A}^{k}$, the symbolic trace $\operatorname{tr}=\mathrm{e}_{1}^{\text {sid }} ; \ldots ; \mathrm{e}_{n}^{\text {sid }}$ associated to sc and $\alpha$ is defined as follows.

Let $q_{i}=\#\left\{j \mid j \leq i,\left(r_{j}, s_{i d}\right) \in \mathrm{sc}\right.$, and $\left.\operatorname{sid}_{j}=\operatorname{sid}_{i}\right\}$, i.e. the number of occurrences up to this point in sc of the session $\operatorname{sid}_{i}$. We have that $q_{i} \leq \ell_{r_{i}}$ and $\mathrm{e}_{i}=\left(\mathrm{e}_{q_{i}}^{r_{i}}\right) \sigma_{r_{i}, s i d_{i}}$, where $\operatorname{dom}\left(\sigma_{r, s i d}\right)=\operatorname{vars}(\Pi(r))$ and

- $\sigma_{r, s i d}(y)=n_{y}^{\text {sid }}$ if $y \in\left\{y_{1}^{r}, \ldots, y_{p_{r}}^{r}\right\}$, where $n_{y}^{\text {sid }}$ is a fresh name from $\mathcal{N}$;

- $\sigma_{r, s i d}\left(x_{i}^{r}\right)=a_{i}$ when $\alpha($ sid $)=\left(a_{1}, \ldots, a_{k}\right)$;

- $\sigma_{r, s i d}(z)=z^{\text {sid }}$ otherwise, where $z^{\text {sid }}$ is a fresh variable.

A session sid is said to be dishonest w.r.t. $\alpha$ and a set of ground atoms $T_{0}$ when $\alpha($ sid $)=$ $\left(a_{1}, \ldots, a_{k}\right)$ and $T_{0} \vdash \operatorname{priv}\left(a_{i}\right)$ or $T_{0} \vdash \operatorname{shk}\left(a_{i}, v\right)$ for some $v \neq \epsilon$ and $1 \leq i \leq k$.

Intuitively, a session sid is honest if all of its participants, from the point of view of the agent playing the session sid, are honest (i.e. they are neither the attacker $\epsilon$ nor did they disclose their long-term keys). Note that since all agent variables occurring in a role, occur as parameters of this role (see Definition [3.2), a symbolic trace does not contain agent variables.

The notational conventions we use for names and variables occurring in a symbolic trace $\left(e . g . n_{y}^{\text {sid }}\right.$ and $z^{\text {sid }}$ ) are not really relevant to state our main result. However, we will rely on this notation in Part II when we prove our reduction result.

Example 3.6. Consider again the Needham-Schroeder protocol. Let $\Pi(1)$ and $\Pi(2)$ be the two roles introduced in Example 3.3. Let $s_{1}$ and $s_{2}$ be two sessions numbers $\left(s_{1} \neq\right.$ $\left.s_{2}\right)$, sc $=\left(1, s_{1}\right)\left(2, s_{2}\right)\left(2, s_{2}\right)\left(1, s_{1}\right)\left(1, s_{1}\right)$ and $\alpha$ the function such that $\operatorname{dom}(\alpha)=\left\{s_{1}, s_{2}\right\}$, $\alpha\left(s_{1}\right)=(a, c)$, and $\alpha\left(s_{2}\right)=(a, b)$. This is the scenario allowing us to retrieve the famous attack due to Lowe 24]. The symbolic trace associated to $\Pi, \mathrm{sc}$, and $\alpha$ is given below:

$$
\begin{aligned}
\operatorname{tr}= & \operatorname{snd}\left(\operatorname{enca}\left(\left\langle n_{y}^{s_{1}}, a\right\rangle, \operatorname{pub}(c)\right)\right) ; \\
& \operatorname{rcv}\left(\operatorname{enca}\left(\left\langle z^{s_{2}}, a\right\rangle, \operatorname{pub}(b)\right)\right) ; \operatorname{snd}\left(\operatorname{enca}\left(\left\langle z^{s_{2}}, n_{y^{\prime}}^{s_{2}}\right\rangle, \operatorname{pub}(a)\right)\right) ; \\
& \operatorname{rcv}\left(\operatorname{enca}\left(\left\langle n_{y}^{s_{1}}, z^{s_{1}}\right\rangle, \operatorname{pub}(a)\right)\right) ; \operatorname{snd}\left(\operatorname{enca}\left(z^{s_{1}}, \operatorname{pub}(c)\right)\right)
\end{aligned}
$$

An execution trace is an instance of such a symbolic trace. Appending an event e to an execution trace exec is written exec; e. The function length has the usual meaning: length $([])=0$ and length(exec; e) $=1+$ length(exec). The prefix of an execution trace consisting of the first $i$ events is denoted as $\operatorname{exec}_{i}$, with $\operatorname{exec}_{0}=[]$ and $\operatorname{exec}_{n}=\operatorname{exec}$ when $n \geq$ length (exec).

Definition 3.7 (knowledge of an execution trace exec). Let exec be an execution trace. The knowledge of exec is the set of terms given by $\mathrm{K}(\mathrm{exec})=\{u \mid \operatorname{snd}(u) \in \operatorname{exec}\}$.

As usual, we are only interested in valid execution traces - those traces where the attacker only sends messages that he can compute from his initial knowledge and the messages he has seen on the network.

Definition 3.8 (valid execution trace). Let $T_{0}$ be a set of ground terms (intuitively $T_{0}$ represents the initial knowledge of the attacker). A ground execution trace exec $=\mathrm{e}_{1}^{\text {sid }_{1}} ; \ldots ; \mathrm{e}_{\ell} \mathrm{sid}_{\ell}$ is valid w.r.t. $T_{0}$ if for all $1 \leq i \leq \ell$, whenever $\mathrm{e}_{i}=\operatorname{rcv}(m)$, we have that $T_{0} \cup \mathrm{K}\left(\operatorname{exec}_{i}\right) \vdash m$.

Example 3.9. Let $T_{0}=\{a, b, c, \operatorname{priv}(c)\}$. Let $\operatorname{tr}$ be the symbolic trace described in Example 3.6 and $\sigma=\left\{z^{s_{1}} \mapsto n_{y^{\prime}}^{s_{2}}, z^{\prime s_{2}} \mapsto n_{y}^{s_{1}}\right\}$. The execution trace $\operatorname{tr} \sigma$ is valid w.r.t. $T_{0}$. Indeed, we have that 
- $T_{1} \stackrel{\text { def }}{=} T_{0} \cup\left\{\operatorname{enca}\left(\left\langle n_{y}^{s_{1}}, a\right\rangle, \operatorname{pub}(c)\right)\right\} \vdash \operatorname{enca}\left(\left\langle n_{y}^{s_{1}}, a\right\rangle, \operatorname{pub}(b)\right)$, and - $T_{1} \cup\left\{\operatorname{enca}\left(\left\langle n_{y}^{s_{1}}, n_{y^{\prime}}^{s_{2}}\right\rangle, \operatorname{pub}(a)\right)\right\} \vdash \operatorname{enca}\left(\left\langle n_{y}^{s_{1}}, n_{y^{\prime}}^{s_{2}}\right\rangle, \operatorname{pub}(a)\right)$.

The purpose of the following lemma is to characterize the terms that occur in plaintext position in a valid execution. Intuitively, the lemma states that any plaintext occurring in a valid execution either occurs as a plaintext in the underlying symbolic trace, or was known by the attacker since the beginning, i.e., is part of the attacker's initial knowledge.

Lemma 3.10. Let $\Pi$ be a $k$-party protocol and $\mathrm{tr}=\left[\mathrm{ee}_{1}^{s i d_{1}} ; \ldots ; \mathrm{ee}_{\ell}^{\text {sid }_{\ell}}\right]$ be a symbolic trace associated to it. Let $T_{0}$ be a set of ground atoms, and exec $\left.=\left[\mathrm{e}_{1}^{\text {sid }}{ }_{1} ; \ldots ; \mathrm{e}_{\ell}^{\text {sid }}\right]_{\ell}\right]$ be a valid execution trace associated to $\operatorname{tr}$ (w.r.t. $T_{0}$ ). We have that:

$$
\text { plaintext (exec) } \subseteq \text { plaintext }(\operatorname{tr}) \cup T_{0} \cup \mathcal{N}_{\epsilon} \cup \mathcal{K}_{\epsilon} \cup \mathcal{A} \cup\{\operatorname{pub}(a) \mid a \in \mathcal{A}\} .
$$

This lemma can be shown by induction on the length of the underlying symbolic trace. We rely on Lemma 2.4 to deal with the case of a receive event, and on the plaintext origination property (Condition (2) in Definition 3.2) to deal with the case of a status or a send event.

\section{SECURITY PROPERTIES}

In this section, we propose a logic for specifying security properties. Our logic is similar to existing ones (see e.g [16, 17]). In particular, it is expressive enough to specify security properties like secrecy and different forms of authentication including aliveness, weak agreement and non-injective agreement. Its semantics is defined as usual on execution traces.

4.1. A logic for security properties. As in [17], status events are used to specify security properties while the other events describe the execution of the protocol. We only consider one temporal operator and this operator should only concern status events. That is why we divide the logic into two layers.

Definition 4.1. A formula of $\mathcal{L}$ is an expression $\phi$ defined by the following grammar:

$$
\begin{aligned}
\phi, \phi_{i} & :=\operatorname{learn}\left(u_{0}\right)|\neg \phi| \exists x . \phi\left|\phi_{1} \vee \phi_{2}\right| \mathrm{C}(u)|\diamond \psi| \psi \\
\psi, \psi_{i} & :=\operatorname{true}\left|\mathrm{P}\left(u_{1}, \ldots, u_{n}\right)\right| \neg \psi \mid \psi_{1} \vee \psi_{2}
\end{aligned}
$$

where $u_{0}, u_{1}, \ldots, u_{n}$ are terms and $u \in \mathcal{A} \cup \mathcal{X}$.

Standard formulas true, $\neg \phi$, and $\phi_{1} \vee \phi_{2}$ carry the usual meaning. The formula learn $\left(u_{0}\right)$ states that the attacker knows the term $u_{0}$, whereas $\mathrm{P}\left(u_{1}, \ldots, u_{n}\right)$ is a status event. The formula $\mathrm{C}(u)$ states that the agent $u$ is compromised (his secret keys are known to the attacker). The formula $\diamond \psi$ means that ' $\psi$ held in the past'. When $x$ is a variable, we write $\exists x . \phi$ to bind $x$ in $\phi$, with the quantifier carrying the usual meaning. Other operators can be represented using the above defined operators. For instance, the abbreviations $\mathrm{NC}(u)$, false, $\wedge, \forall$, and $\Rightarrow$ are defined by $\mathrm{NC}(u) \stackrel{\text { def }}{=} \neg \mathrm{C}(u)$, false $\stackrel{\text { def }}{=} \neg$ true, $\phi_{1} \wedge \phi_{2} \stackrel{\text { def }}{=} \neg\left(\neg \phi_{1} \vee \neg \phi_{2}\right)$, $\forall x . \phi \stackrel{\text { def }}{=} \neg \exists x . \neg \phi$, and $\phi_{1} \Rightarrow \phi_{2} \stackrel{\text { def }}{=} \neg \phi_{1} \vee \phi_{2}$.

In the sequel, we assume that formulas are closed, i.e. they contain no free variables, and that each variable is quantified at most once (this can be easily ensured by using renaming). We also assume that the variables occurring in a formula $\phi$ are disjoint from the variables occurring in the considered symbolic trace. 
Formulas are interpreted at some position along an execution trace as stated in Definition 4.2.

Definition 4.2 (concrete validity). Let $\phi$ be a closed formula in $\mathcal{L}$, exec be a ground execution trace and $T_{0}$ be a set of ground terms. We define $\left\langle\right.$ exec, $\left.T_{0}\right\rangle \models \phi$ as:

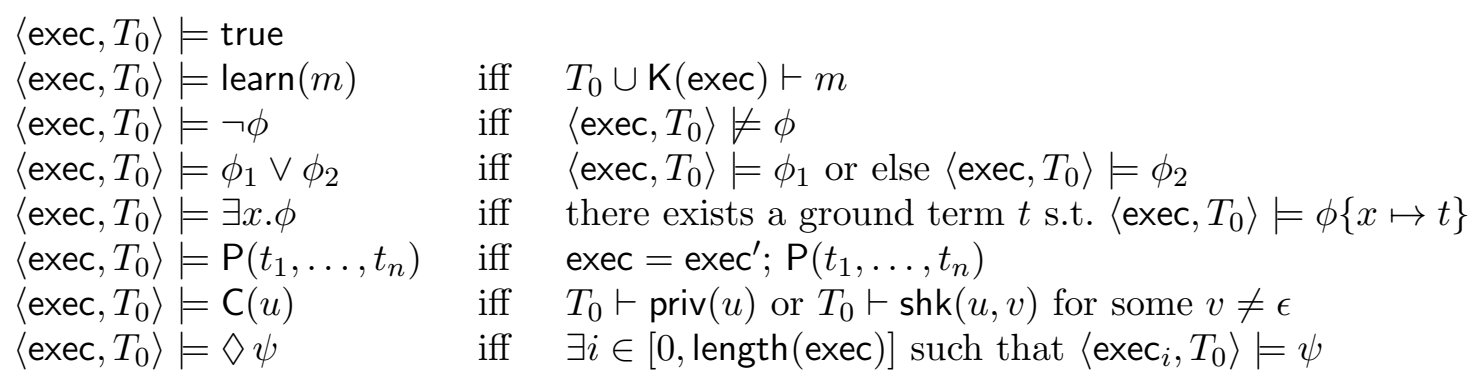

Given a protocol $\Pi$ and a set of ground terms $T_{0}$, we say that $\Pi \models \phi$ w.r.t. $T_{0}$, if $\left\langle\right.$ exec, $\left.T_{0}\right\rangle \models$ $\phi$ for all valid execution traces exec of $\Pi$ w.r.t. $T_{0}$.

We now define the subset of $\mathcal{L}$ for which our result holds. We say a formula in $\mathcal{L}$ is quantifier-free if it does not contain any $\exists$. A formula is modality-free if it does not contain any $\diamond$. We will only consider attack formulas of the form $\exists x_{1} \ldots . \exists x_{n}$. $\phi^{\prime}$ where $\phi^{\prime}$ is quantifier-free, and we consider also some additional syntactic restrictions. Therefore, the security formulas we consider are of the form $\forall x_{1}, \ldots, \forall x_{n} . \neg \phi^{\prime}$, i.e. the negation of an attack formula.

Definition 4.3 (attack formula). An attack formula is an expression of the form

$$
\exists x_{1} \ldots \exists x_{n} . \phi
$$

where all the variables $x_{i}$ are distinct and $\phi$ is a quantifier-free formula of $\mathcal{L}$ satisfying the following conditions:

(1) all subterms of $\phi$ are atomic terms with no names, i.e. St $(\phi) \subseteq \mathcal{A} \cup \mathcal{X} \cup \mathcal{Y}$,

(2) for any term $t$, learn $(t)$ can only occur positively in $\phi$, i.e. under an even number of negations,

(3) any variable occurs at most once in a positive status event,

(4) if $\diamond \psi$ is a subformula of $\phi$ that occurs negatively in $\phi$, then a status event can only occur positively in $\psi$.

As we will see next this fragment is expressive enough to model classical security properties.

4.2. Some security properties. We now show how classical security properties like secrecy and several flavors of non-injective authentication properties can be expressed in our logic.

4.2.1. Secrecy. The secrecy property is the inability of the intruder to learn a message (e.g. a nonce, a key, or a compound term) that is specified (using a status event) as confidential. We will show how to specify the secrecy property for a nonce for example with a formula in $\mathcal{L}$. Let $\Pi$ be a $k$-party protocol with

$$
\Pi(j)=\lambda x_{1}^{j} \ldots \lambda x_{k}^{j} . \nu y_{1}^{j} \ldots \nu y_{p_{j}}^{j} \cdot \mathrm{e}_{1}^{j} ; \ldots ; \mathrm{e}_{\ell_{j}}^{j} \quad \text { for } 1 \leq j \leq k .
$$


and let $y_{h}^{j}\left(1 \leq j \leq k\right.$ and $1 \leq h \leq p_{j}$ ) be the nonce variable whose instantiations should remain confidential. In order to specify that all the instances of $y_{h}^{j}$ must remain secret we build from $\Pi$, a protocol $\Pi_{S}$ as follows. Let Secret be a predicate not occurring in $\Pi$, then

$$
\Pi_{\mathrm{S}}(n)= \begin{cases}\Pi(n) & \text { for } 1 \leq n \leq k \\ \lambda x_{1}^{j} \ldots \lambda x_{k}^{j} . \nu y_{1}^{j} \ldots \nu y_{p_{j}}^{j} \cdot \operatorname{Secret}\left(x_{1}^{j}, \ldots, x_{k}^{j}, y_{h}^{j}\right) ; \mathrm{e}_{1}^{j} ; \ldots ; \mathrm{e}_{\ell_{j}} \quad \text { for } n=j\end{cases}
$$

During an execution, the predicate Secret will link each instance $n_{y_{h}^{j}}^{\text {sid }}$ of $y_{h}^{j}$ to the participants of the corresponding session sid. The following property expresses that the noncompromised instances of $y_{h}^{j}$ should remain confidential

$\phi_{\mathrm{S}}=\forall x_{1} \ldots . \forall x_{k} . \forall y .\left[\left(\left(\diamond \operatorname{Secret}\left(x_{1}, \ldots, x_{k}, y\right)\right) \wedge \mathrm{NC}\left(x_{1}\right) \wedge \ldots \wedge \mathrm{NC}\left(x_{k}\right)\right) \Rightarrow \neg\right.$ learn $\left.(y)\right]$.

And the following formula is the corresponding attack formula

$$
\overline{\phi_{\mathrm{S}}}=\exists x_{1} \ldots . \exists x_{k} \cdot \exists y .\left[\left(\diamond \operatorname{Secret}\left(x_{1}, \ldots, x_{k}, y\right)\right) \wedge \mathrm{NC}\left(x_{1}\right) \wedge \ldots \wedge \mathrm{NC}\left(x_{k}\right) \wedge \text { learn }(y)\right] .
$$

which satisfies the 4 conditions of the definition of an attack formula (Definition 4.3). Note that the same construction can be used to model the secrecy of a compound term $t$ as seen by the agent executing the role $\Pi(j)$. For this, we simply add a status event $\operatorname{Secret}\left(x_{1}^{j}, \ldots, x_{k}^{j}, t\right)$ in $\Pi(j)$, and keep the attack formula unchanged. The 4 conditions stated in Definition 4.3 are still satisfied.

Example 4.4. Let us come back to the Needham-Schroeder protocol as presented in Example 3.3 to illustrate this property, and let's specify that the nonce $y^{\prime}$ generated by the responder is confidential. In order to do so, we build the 2-party protocol $\Pi_{\mathrm{S}}$ following the above mentioned construction, i.e. such that $\Pi_{S}(1)=\Pi(1)$, and

$$
\begin{aligned}
\Pi_{\mathrm{S}}(2)= & \lambda x_{A} \cdot \lambda x_{B} \cdot \nu y^{\prime} . \\
& \operatorname{Secret}\left(x_{A}, x_{B}, y^{\prime}\right) \\
& \operatorname{rcv}\left(\operatorname{enca}\left(\left\langle z^{\prime}, x_{A}\right\rangle, \operatorname{pub}\left(x_{B}\right)\right)\right) ; \\
& \operatorname{snd}\left(\operatorname{enca}\left(\left\langle z^{\prime}, y^{\prime}\right\rangle, \operatorname{pub}\left(x_{A}\right)\right)\right) ; \\
& \operatorname{rcv}\left(\operatorname{enca}\left(y^{\prime}, \operatorname{pub}\left(x_{B}\right)\right)\right)
\end{aligned}
$$

An attack on the secrecy of $y^{\prime}$, is any valid execution trace of $\Pi_{S}$ that reveals to the intruder an honest instance of $y^{\prime}$ (i.e. generated by an honest session of $\Pi_{\mathrm{S}}(2)$ ). Formally, an attack on the secrecy of $y^{\prime}$ is a valid execution trace of $\Pi_{S}$ that satisfies the following attack formula

$$
\overline{\phi_{\mathrm{S}}}=\exists y_{A} \cdot \exists y_{B} \cdot \exists x .\left[\left(\diamond \operatorname{Secret}\left(y_{A}, \ldots, y_{B}, x\right)\right) \wedge \mathrm{NC}\left(y_{A}\right) \wedge \mathrm{NC}\left(y_{B}\right) \wedge \operatorname{learn}(x)\right] .
$$

Let us consider as initial intruder knowledge $T_{0}=\{a, b, c$, priv $(c)\}$, the scenario sc $=$ $\left(1, s_{1}\right)\left(2, s_{2}\right)\left(2, s_{2}\right)\left(2, s_{2}\right)\left(1, s_{1}\right)\left(1, s_{1}\right)$, and the function $\alpha$ such that $\operatorname{dom}(\alpha)=\left\{s_{1}, s_{2}\right\}$, $\alpha\left(s_{1}\right)=(a, c)$, and $\alpha\left(s_{2}\right)=(a, b)$. We denote by tr the symbolic trace associated to $\Pi_{\mathrm{S}}$, sc, and $\alpha$. Let $\sigma$ be the substitution such that $\sigma=\left\{z^{s_{1}} \mapsto n_{y^{\prime}}^{s_{2}}, z^{\prime s_{2}} \mapsto n_{y}^{s_{1}}\right\}$. The execution $\operatorname{trace} \operatorname{tr} \sigma$ is valid w.r.t. $T_{0}$. This execution corresponds to the famous attack due to Lowe [24], and formally satisfies $\overline{\phi_{\mathrm{S}}}$, i.e. $\left\langle T_{0}, \operatorname{tr} \sigma\right\rangle \models \overline{\phi_{\mathrm{S}}}$, and thus $\Pi_{\mathrm{S}} \forall \phi_{\mathrm{S}}$ w.r.t. $T_{0}$.

We are now going to look at how to formally express authentication properties. 
4.2.2. Aliveness. We start with the weakest notion of authentication in the hierarchy of Lowe [25], namely aliveness. Informally, a protocol $\Pi$ satisfies aliveness if and only if each time a participant $a$ finishes an honest session involving participant $b$ (of any of the roles of $\Pi$ ), $b$ has at least partially executed one session (of any of the roles of $\Pi$ ), and in that sense $b$ is alive.

In order to express this property, we need to detect in the executions of $\Pi$, each time a session starts and ends. This can be achieved by adding status events at the beginning and the end of each role. More precisely, if we consider the $k$-party protocol $\Pi$ with

$$
\Pi(j)=\lambda x_{1}^{j} \ldots \lambda x_{k}^{j} . \nu y_{1}^{j} \ldots \nu y_{p_{j}}^{j} \cdot \mathrm{e}_{1}^{j} ; \ldots ; \mathrm{e}_{\ell_{j}}^{j} \quad \text { for } 1 \leq j \leq k .
$$

We build the protocol $\Pi_{\mathrm{A}}$ by inserting new status events as follows:

$$
\Pi_{\mathrm{A}}(j)=\lambda x_{1}^{j} \ldots \lambda x_{k}^{j} . \nu y_{1}^{j} \ldots \nu y_{p_{j}}^{j} \cdot \operatorname{Start}\left(x_{j}^{j}\right) ; \mathrm{e}_{1}^{j} ; \ldots ; \mathrm{e}_{\ell_{j}}^{j} ; \operatorname{End}\left(x_{1}^{j}, \ldots, x_{k}^{j}\right) \quad \text { for } 1 \leq j \leq k .
$$

where the predicates Start and End will mark in an execution the beginning and the end of each session, and will link together the effective participants of each session. Aliveness can then be modelled by the following formula

$$
\phi_{\mathrm{A}}=\left\{\begin{aligned}
\forall y_{1} \ldots \forall y_{k} \cdot\left[\operatorname{End}\left(y_{1}, \ldots, y_{k}\right) \wedge \mathrm{NC}\left(y_{1}\right)\right. & \wedge \wedge \mathrm{NC}\left(y_{k}\right) \\
& \left.\Rightarrow \diamond \operatorname{Start}\left(y_{1}\right) \wedge \ldots \wedge \Delta \operatorname{Start}\left(y_{k}\right)\right]
\end{aligned}\right.
$$

An attack on protocol $\Pi$ w.r.t. aliveness is thus a trace of $\Pi_{\mathrm{A}}$ satisfying the following attack formula

$$
\overline{\phi_{\mathrm{A}}}=\left\{\begin{aligned}
\exists y_{1} \ldots \exists y_{k} .\left[\operatorname{End}\left(y_{1}, \ldots, y_{k}\right) \wedge \mathrm{NC}\left(y_{1}\right)\right. & \wedge \ldots \wedge \mathrm{NC}\left(y_{k}\right) \\
& \left.\wedge\left(\neg \diamond \operatorname{Start}\left(y_{1}\right) \vee \ldots \vee \neg \diamond \operatorname{Start}\left(y_{k}\right)\right)\right]
\end{aligned}\right.
$$

Example 4.5. Let us come back to the Needham-Schroeder protocol as presented in Example 3.3 to illustrate this property. In order to do so, we build the 2-party protocol $\Pi_{\mathrm{A}}$ following the above mentioned construction, i.e. such that

$$
\begin{array}{rlrl}
\Pi_{\mathrm{A}}(1)= & \lambda x_{A} \cdot \lambda x_{B} \cdot \nu y . & \Pi_{\mathrm{A}}(2)= & \lambda x_{A} \cdot \lambda x_{B} \cdot \nu y^{\prime} . \\
& \operatorname{Start}\left(x_{A}\right) & \operatorname{Start}\left(x_{B}\right) \\
& \operatorname{snd}\left(\operatorname{enca}\left(\left\langle y, x_{A}\right\rangle, \operatorname{pub}\left(x_{B}\right)\right)\right) ; & \operatorname{rcv}\left(\operatorname{enca}\left(\left\langle z^{\prime}, x_{A}\right\rangle, \operatorname{pub}\left(x_{B}\right)\right)\right) ; \\
& \operatorname{rcv}\left(\operatorname{enca}\left(\langle y, z\rangle, \operatorname{pub}\left(x_{A}\right)\right)\right) ; & \operatorname{snd}\left(\operatorname{enca}\left(\left\langle z^{\prime}, y^{\prime}\right\rangle, \operatorname{pub}\left(x_{A}\right)\right)\right) ; \\
\operatorname{snd}\left(\operatorname{enca}\left(z, \operatorname{pub}\left(x_{B}\right)\right)\right) & \operatorname{rcv}\left(\operatorname{enca}\left(y^{\prime}, \operatorname{pub}\left(x_{B}\right)\right)\right) \\
\operatorname{End}\left(x_{A}, x_{B}\right) & & \operatorname{End}\left(x_{A}, x_{B}\right)
\end{array}
$$

Now this protocol satisfies aliveness, if in every valid execution trace of $\Pi_{A}$ during which an agent $a$ executing an honest session of role $\Pi_{\mathrm{A}}(1)\left(\right.$ resp. $\left.\Pi_{\mathrm{A}}(2)\right)$ with agent $b, b$ has also initiated a session of the protocol. Formally, $\Pi$ satisfies aliveness if every valid execution trace of $\Pi_{\mathrm{A}}$ satisfies the following formula;

$$
\phi_{\mathrm{A}}=\forall x_{A} . \forall x_{B} . \operatorname{End}\left(x_{A}, x_{B}\right) \wedge \mathrm{NC}\left(x_{A}\right) \wedge \mathrm{NC}\left(x_{B}\right) \Rightarrow\left[\diamond \operatorname{Start}\left(x_{A}\right) \wedge \diamond \operatorname{Start}\left(x_{B}\right)\right]
$$

Consider the symbolic trace $\operatorname{tr}$ associated to the scenario

$$
\mathrm{sc}_{\mathrm{A}}=\left(1, s_{1}\right)\left(1, s_{1}\right)\left(2, s_{2}\right)\left(2, s_{2}\right)\left(2, s_{2}\right)\left(1, s_{1}\right)\left(1, s_{1}\right)\left(1, s_{1}\right)\left(2, s_{2}\right)\left(2, s_{2}\right)
$$

and the function $\alpha$ as defined in Example 4.4. Actually, we have that $\left\langle T_{0}, \operatorname{tr} \sigma\right\rangle \models \phi_{\mathrm{A}}$ using the set $T_{0}$ and the substitution $\sigma$ as defined in Example 4.4. More generally, using an automatic tool such as ProVerif [8, one can prove that the Needham-Schroeder protocol satisfies aliveness w.r.t. the initial intruder knowledge $T_{0}=\{a, b, c, \operatorname{priv}(c)\}$, i.e. $\Pi_{\mathrm{A}} \models \phi_{\mathrm{A}}$ w.r.t. $T_{0}$. 
4.2.3. Weak agreement. Weak agreement is slightly stronger than aliveness. Informally, a protocol $\Pi$ satisfies weak agreement, if and only if each time a participant $a$ finishes an honest session involving participant $b$ (of any of the roles of $\Pi$ ), $b$ has at least initiated a session involving $a$ (of any of the roles of $\Pi$ ).

Again, in order to express this property, we need to detect in the executions of $\Pi$, each time a session starts and ends, but also which participants are involved in each session that is initiated. This can be achieved by adding status events at the beginning and the end of each role. More precisely, if we consider the $k$-party protocol $\Pi$ with

$$
\Pi(j)=\lambda x_{1}^{j} \ldots \lambda x_{k}^{j} . \nu y_{1}^{j} \ldots \nu y_{p_{j}}^{j} \cdot \mathrm{e}_{1}^{j} ; \ldots ; \mathrm{e}_{\ell_{j}}^{j} \quad \text { for } 1 \leq j \leq k .
$$

We build the protocol $\Pi_{\mathrm{WA}}$ by inserting new status events as follows:

$$
\begin{array}{r}
\Pi_{\mathrm{WA}}(j)=\lambda x_{1}^{j} \ldots \lambda x_{k}^{j} . \nu y_{1}^{j} \ldots \nu y_{p_{j}}^{j} \cdot \operatorname{Start}\left(x_{j}^{j}, x_{1}^{j}\right) ; \ldots ; \operatorname{Start}_{j, k}\left(x_{j}^{j}, x_{k}^{j}\right) ; \\
\mathrm{e}_{1}^{j} ; \ldots ; \mathrm{e}_{\ell_{j}}^{j} ; \operatorname{End}_{j}\left(x_{1}^{j}, \ldots, x_{k}^{j}\right) \quad \text { for } 1 \leq j \leq k .
\end{array}
$$

where the predicates Start and End will mark in an execution the beginning and the end of each session, and will link together the effective participants of each session both at the beginning and the end of the session. Weak agreement can then be modelled by the following formula

$$
\begin{gathered}
\phi_{\mathrm{WA}}=\forall y_{1}^{1} \ldots \forall y_{k}^{1} \ldots \forall y_{1}^{k} \ldots \forall y_{k}^{k} . \\
\bigwedge_{j \in\{1, \ldots, k\}}\left[\operatorname{End}_{j}\left(y_{1}^{j}, \ldots, y_{k}^{j}\right) \wedge \mathrm{NC}\left(y_{1}^{j}\right) \wedge \ldots \wedge \mathrm{NC}\left(y_{k}^{j}\right) \Rightarrow \bigwedge_{i \in\{1, \ldots, k\}, i \neq j} \nabla \operatorname{Start}\left(y_{i}^{j}, y_{j}^{j}\right)\right]
\end{gathered}
$$

An attack on protocol $\Pi$ w.r.t. aliveness is thus a trace of $\Pi_{W A}$ satisfying the following attack formula

$$
\overline{\phi_{\mathrm{WA}}} \equiv \exists y_{1} \ldots \exists y_{k} . \operatorname{End}_{j}\left(y_{1}, \ldots, y_{k}\right) \wedge \mathrm{NC}\left(y_{1}\right) \wedge \ldots \wedge \mathrm{NC}\left(y_{k}\right) \wedge \neg \diamond \operatorname{Start}\left(y_{i}, y_{j}\right)
$$

for some $j, i \in\{1, \ldots, k\}$ with $i \neq j$.

Example 4.6. Let us come back to the Needham-Schroeder protocol as presented in Example 3.3 to illustrate this property. In order to do so, we build the 2-party protocol ПWA following the above mentioned construction, i.e. such that:

$$
\begin{aligned}
& \Pi_{\mathrm{WA}}(1)=\lambda x_{A} \cdot \lambda x_{B} \cdot \nu y \text {. } \\
& \operatorname{Start}\left(x_{A}, x_{A}\right) \\
& \operatorname{Start}\left(x_{A}, x_{B}\right) \\
& \operatorname{snd}\left(\operatorname{enca}\left(\left\langle y, x_{A}\right\rangle, \operatorname{pub}\left(x_{B}\right)\right)\right) \text {; } \\
& \operatorname{rcv}\left(\operatorname{enca}\left(\langle y, z\rangle, \operatorname{pub}\left(x_{A}\right)\right)\right) \text {; } \\
& \operatorname{snd}\left(\operatorname{enca}\left(z, \operatorname{pub}\left(x_{B}\right)\right)\right) \\
& \operatorname{End}_{1}\left(x_{A}, x_{B}\right) \\
& \begin{aligned}
\Pi_{\mathrm{WA}}(2)= & \lambda x_{A} \cdot \lambda x_{B} \cdot \nu y^{\prime} . \\
& \operatorname{Start}\left(x_{B}, x_{A}\right) \\
& \operatorname{Start}\left(x_{B}, x_{B}\right) \\
& \operatorname{rcv}\left(\operatorname{enca}\left(\left\langle z^{\prime}, x_{A}\right\rangle, \operatorname{pub}\left(x_{B}\right)\right)\right) ; \\
& \operatorname{snd}\left(\operatorname{enca}\left(\left\langle z^{\prime}, y^{\prime}\right\rangle, \operatorname{pub}\left(x_{A}\right)\right)\right) ; \\
& \operatorname{rcv}\left(\operatorname{enca}\left(y^{\prime}, \operatorname{pub}\left(x_{B}\right)\right)\right) \\
& \operatorname{End}_{2}\left(x_{A}, x_{B}\right)
\end{aligned}
\end{aligned}
$$

Now this protocol satisfies weak agreement, if in every valid execution trace of ПWWA during which an agent $a$ executing an honest session of role $\Pi_{\mathrm{WA}}(1)\left(\right.$ resp. $\left.\Pi_{\mathrm{WA}}(2)\right)$ with agent $b, b$ has also initiated a session of the protocol involving agent $a$. In other words, $\Pi$ admits an attack w.r.t. weak agreement if there exists a valid execution trace of $\Pi_{W A}$ that 
satisfies the following formula:

$$
\begin{gathered}
\overline{\phi_{\mathrm{WA}}} \equiv \exists x_{A}^{1} \cdot \exists x_{B}^{1} \cdot \exists x_{A}^{2} \cdot \exists x_{B}^{2} . \\
{\left[\begin{array}{rl}
\operatorname{End}_{1}\left(x_{A}^{1}, x_{B}^{1}\right) \wedge \mathrm{NC}\left(x_{A}^{1}\right) & \wedge \mathrm{NC}\left(x_{B}^{1}\right) \wedge \neg \diamond \operatorname{Start}\left(x_{B}^{1}, x_{A}^{1}\right) \\
\operatorname{End}_{2}\left(x_{A}^{2}, x_{B}^{2}\right) & \wedge \mathrm{NC}\left(x_{A}^{2}\right) \wedge \mathrm{NC}\left(x_{B}^{2}\right) \wedge \neg \diamond \operatorname{Start}\left(x_{A}^{2}, x_{B}^{2}\right)
\end{array}\right]}
\end{gathered}
$$

Let's consider as initial intruder knowledge $T_{0}=\{a, b, c$, priv $(c)\}$, the scenario

$$
\mathrm{sc}=\left(1, s_{1}\right)\left(1, s_{1}\right)\left(1, s_{1}\right)\left(2, s_{2}\right)\left(2, s_{2}\right)\left(2, s_{2}\right)\left(2, s_{2}\right)\left(1, s_{1}\right)\left(1, s_{1}\right)\left(1, s_{1}\right)\left(2, s_{2}\right)\left(2, s_{2}\right)\left(2, s_{2}\right),
$$

the function $\alpha$ such that $\operatorname{dom}(\alpha)=\left\{s_{1}, s_{2}\right\}, \alpha\left(s_{1}\right)=(a, c)$, and $\alpha\left(s_{2}\right)=(a, b)$, and the substitution $\sigma=\left\{z^{s_{1}} \mapsto n_{y^{\prime}}^{s_{2}}, z^{\prime s_{2}} \mapsto n_{y}^{s_{1}}\right\}$. The execution trace $\operatorname{tr} \sigma$ is valid w.r.t. $T_{0}$ with $\operatorname{tr}$ the symbolic trace associated to sc and $\alpha$. This execution corresponds to the famous attack due to Lowe [24], and formally satisfies $\overline{\phi_{\mathrm{WA}}}$, i.e. $\left\langle T_{0}, \operatorname{tr} \sigma\right\rangle \models \overline{\phi_{\mathrm{WA}}}$, and thus $\Pi_{\mathrm{WA}} \not \models \phi_{\mathrm{WA}}$ w.r.t. $T_{0}$.

\section{Transformation OF PROTOCOLS}

In Section 5.1 we define our transformation before we state our main result in Section 5.2 whose proof is postponed to Part II.

5.1. Our transformation. Given an input protocol $\Pi$, our transformation will compute a new protocol $\widetilde{\Pi}$ which consists in two phases. During the first phase, the protocol participants try to agree on some common, dynamically generated, session identifier $\tau$. For this, each participant sends a freshly generated nonce $N_{i}$ together with his identity $A_{i}$ to all other participants. (Note that if broadcast is not practical or if not all identities are known to each participant, the message can be sent to some of the participants who forwards the message.) At the end of this preamble, each participant computes a session identifier: $\tau=\left\langle\left\langle A_{1}, N_{1}\right\rangle, \ldots,\left\langle A_{k}, N_{k}\right\rangle\right\rangle$. Note that an active attacker may interfere with this initialization phase and may intercept and replace some of the nonces. Hence, the protocol participants do not necessarily agree on the same session identifier $\tau$ after this preamble. In fact, each participant computes his own session identifier, say $\tau_{j}$. During the second phase, each participant $j$ executes the original protocol in which the dynamically computed identifier is used for tagging each application of a cryptographic primitive. In this phase, when a participant opens an encryption, he checks that the tag is in accordance with the nonces he received during the initialization phase. In particular, he can test the presence of his own nonce.

The transformation, using the informal Alice-Bob notation, is described below and relies on the tagging operation that is formally defined in Definition 5.1.

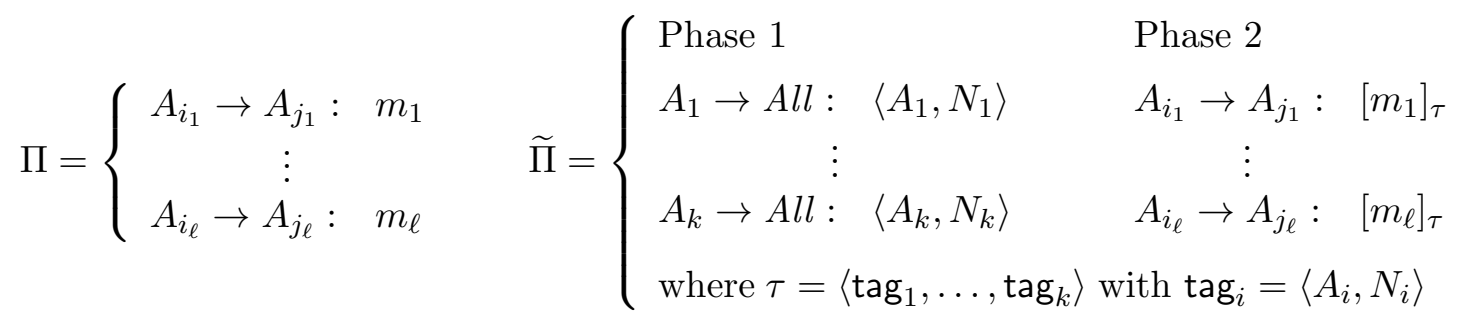


Note that, the Alice-Bob notation only represents what happens in a normal execution, i.e. with no intervention of the attacker. Of course, in such a situation, the participants agree on the same session identifier $\tau$ used in the second phase.

Definition 5.1 ( $k$-tag, $k$-tagging). A $k$-tag is a term $\left\langle\left\langle a_{1}, v_{1}\right\rangle, \ldots,\left\langle a_{k}, v_{k}\right\rangle\right\rangle$ where each $a_{i} \in \mathcal{A}$ and each $v_{i}$ is a term. Let $u$ be a term and tag be a $k$-tag. The $k$-tagging of $u$ with tag, denoted $[u]_{\text {tag }}$, is inductively defined as follows:

$$
\begin{array}{lll}
{\left[\left\langle u_{1}, u_{2}\right\rangle\right]_{\operatorname{tag}}} & =\left\langle\left[u_{1}\right]_{\operatorname{tag}},\left[u_{2}\right]_{\operatorname{tag}}\right\rangle & \\
{\left[\mathrm{f}\left(u_{1}, u_{2}\right)\right]_{\operatorname{tag}}} & =\mathrm{f}\left(\left\langle\operatorname{tag},\left[u_{1}\right]_{\operatorname{tag}}\right\rangle,\left[u_{2}\right]_{\operatorname{tag}}\right) & \text { for } \mathrm{f} \in\{\text { encs, enca, sign }\} \\
{\left[\mathrm{h}\left(u_{1}\right)\right]_{\operatorname{tag}}} & =\mathrm{h}\left(\left\langle\operatorname{tag},\left[u_{1}\right]_{\operatorname{tag}}\right\rangle\right) & \\
{[u]_{\operatorname{tag}}} & =u & \text { otherwise }
\end{array}
$$

We say that a term $t$ is $k$-tagged if $\left.u\right|_{1.1}$ is a $k$-tag for any $u \in \operatorname{CryptSt}(t)$.

These notions are extended to events and sequences of events as expected. We are now able to formally define our transformation.

Definition 5.2 (protocol transformation). Let $\Pi$ be a $k$-party protocol such that

$$
\Pi(j)=\lambda x_{1}^{j} \ldots \lambda x_{k}^{j} \cdot \nu y_{1}^{j} \ldots \nu y_{p_{j}}^{j} \cdot \text { seq }^{j} \quad \text { for } 1 \leq j \leq k .
$$

and the variables $z_{i}^{j}(1 \leq i, j \leq k)$ do not appear in $\Pi$ (which can always be ensured by renaming variables in $\Pi)$. The transformed protocol $\widetilde{\Pi}$ is a $k$-party protocol defined as follows:

$$
\widetilde{\Pi}(j)=\lambda x_{1}^{j} \ldots \lambda x_{k}^{j} \cdot \nu y_{1}^{j} \ldots \nu y_{p_{j}}^{j} \cdot \nu z_{j}^{j} \cdot \widetilde{\Pi}^{\text {init }}(j) ;\left[\mathrm{seq}^{j}\right]_{\tau_{j}} \quad \text { for } 1 \leq j \leq k
$$

where

$$
\widetilde{\Pi}^{\text {init }}(j)=\operatorname{rcv}\left(u_{1}^{j}\right) ; \ldots ; \operatorname{rcv}\left(u_{j-1}^{j}\right) ; \operatorname{snd}\left(u_{j}^{j}\right) ; \operatorname{rcv}\left(u_{j+1}^{j}\right) ; \ldots ; \operatorname{rcv}\left(u_{k}^{j}\right)
$$

and $\tau_{j}=\left\langle u_{1}^{j}, \ldots, u_{k}^{j}\right\rangle$ with $u_{i}^{j}=\left\langle x_{i}^{j}, z_{i}^{j}\right\rangle$.

In the above definition, the protocol $\widetilde{\Pi}^{\text {init }}$ models the initialization phase and the variables $z_{i}^{j}$ correspond to the nonces that are generated and exchanged during this phase. In particular for the role $j$, the variable $z_{j}^{j}$ is a freshly generated nonce while the other variables $z_{i}^{j}, i \neq j$, are expected to be bound to the other participant's nonces in the receive events. Remember also that the variables $x_{i}^{j}$ are the role parameters which correspond to the agents. The tag computed by the $j^{\text {th }}$ role in our transformation consists in the concatenation of the $k$ names of the agents involved in the protocol, together with the $k-1$ terms received during the initialization phase as well as the fresh nonce generated by the role $j$ itself, i.e. $z_{j}^{j}$. We illustrate this transformation on the Needham-Schroeder protocol introduced in Section 2 ,

Example 5.3. Consider the Needham-Schroeder protocol described in Example 3.3. Applying our transformation we obtain a 2-party protocol $\widetilde{\Pi}$. The role $\widetilde{\Pi}(2)$ is described below. The role $\widetilde{\Pi}(1)$ can be obtained in a similar way.

$$
\begin{aligned}
\widetilde{\Pi}(2)= & \lambda x_{A} \lambda x_{B} \cdot \nu y^{\prime} \cdot \nu z_{B} \cdot \operatorname{rcv}\left(\left\langle x_{A}, z_{A}\right\rangle\right) ; \operatorname{snd}\left(\left\langle x_{B}, z_{B}\right\rangle\right) ; \\
& \operatorname{rcv}\left(\operatorname{enca}\left(\left\langle\tau,\left\langle z^{\prime}, x_{A}\right\rangle\right\rangle, \operatorname{pub}\left(x_{B}\right)\right)\right) ; \\
& \operatorname{snd}\left(\operatorname{enca}\left(\left\langle\tau,\left\langle z^{\prime}, y^{\prime}\right\rangle\right\rangle, \operatorname{pub}\left(x_{A}\right)\right)\right) ; \\
& \operatorname{rcv}\left(\operatorname{enca}\left(\left\langle\tau, y^{\prime}\right\rangle, \operatorname{pub}\left(x_{B}\right)\right)\right)
\end{aligned}
$$


where $\tau=\left\langle\left\langle x_{A}, z_{A}\right\rangle,\left\langle x_{B}, z_{B}\right\rangle\right\rangle$. Note that Lowe's famous man-in-the-middle attack [24] described in Example 4.6 does not exist anymore on $\widetilde{\Pi}$.

5.2. Main theorem. Roughly, our result states that if the compiled protocol admits an attack that may involve several sessions, then there exists an attack which only requires a bounded number of sessions of each role, and the bound only depends on the security formula under study. More formally, we define the size of a formula as follows:

Definition 5.4 (size of a formula). Let $\phi$ be a formula. The size of $\phi$, denoted $\|\phi\|$, is defined as follows:

$$
\begin{aligned}
& \begin{aligned}
\| \text { true } \| & \stackrel{\text { def }}{=} 0 \\
\left\|\mathrm{P}\left(t_{1}, \ldots, t_{n}\right)\right\| & \stackrel{\text { def }}{=} 1 \\
\| \text { learn }(t) \| & \stackrel{\text { def }}{=} 0
\end{aligned} \\
& \|\mathrm{C}(t)\| \stackrel{\text { def }}{=} 0 \\
& \|\neg \phi\| \stackrel{\text { def }}{=}\|\phi\|^{-} \\
& \left\|\phi_{1} \vee \phi_{2}\right\| \stackrel{\text { def }}{=} \max \left\{\left\|\phi_{1}\right\|,\left\|\phi_{2}\right\|\right\} \\
& \|\exists x . \phi\| \stackrel{\text { def }}{=}\|\phi\| \\
& \|\diamond \phi\| \stackrel{\text { def }}{=}\|\phi\| \\
& \begin{aligned}
\| \text { true } \|^{-} & \stackrel{\text { def }}{=} 0 \\
\left\|\mathrm{P}\left(t_{1}, \ldots, t_{n}\right)\right\|^{-} & \stackrel{\text { def }}{=} 1 \\
\| \text { learn }(t) \|^{-} & \stackrel{\text { def }}{=} 0 \\
\|\mathrm{C}(t)\|^{-} & \stackrel{\text { def }}{=} 0 \\
\|\neg \phi\|^{-} & \stackrel{\text { def }}{=}\|\phi\| \\
\left\|\phi_{1} \vee \phi_{2}\right\|^{-} & \stackrel{\text { def }}{=}\left\|\phi_{1}\right\|^{-}+\left\|\phi_{2}\right\|^{-} \\
\|\exists x . \phi\|^{-} & \stackrel{\text { def }}{=}\|\phi\|^{-} \\
\|\diamond \phi\|^{-} & \stackrel{\text { def }}{=} 0
\end{aligned}
\end{aligned}
$$

Intuitively, when an attack trace involves several sessions of each role, not all the sessions are necessary to mount the attack. We only need to keep those sessions that witness the satisfiability of the attack formula $\phi$. By definition of an attack formula (see Definition 4.3), we know that each variable occurring in $\phi$ also occurs in a positive status events. Thus, there is no need to take into account the number of occurrences of learn $(t)$ in the previous definition.

Example 5.5. Note that $\left\|\phi_{1} \wedge \phi_{2}\right\|=\left\|\phi_{1}\right\|+\left\|\phi_{2}\right\|$. Considering the attack formulas $\overline{\phi_{\mathrm{S}}}$, $\overline{\phi_{\mathrm{A}}}$, and $\overline{\phi_{\mathrm{WA}}}$ as defined in Section 4.2 , we have that $\left\|\overline{\phi_{\mathrm{S}}}\right\|=\left\|\overline{\phi_{\mathrm{A}}}\right\|=\left\|\overline{\phi_{\mathrm{WA}}}\right\|=1$.

We are now able to state our main transference result.

Theorem 5.6. Let $\Pi$ be a k-party protocol, $\widetilde{\Pi}$ be its corresponding transformed protocol and $T_{0}$ be a set of ground atoms such that lgKeys $(\Pi) \cap$ plaintext $(\Pi) \subseteq T_{0} \cup \mathcal{K}_{\epsilon}$. Let $\phi$ be an attack formula such that $\widetilde{\Pi} \models \phi$ w.r.t. $T_{0}$. There exists a valid execution trace exec of $\widetilde{\Pi}$ such that:

$$
\left\langle\text { exec, } T_{0}\right\rangle \models \phi \text { and exec involves at most }\|\phi\| \text { sessions of each role. }
$$

Applying our result, we can now establish that if a protocol built according to our transformation admits an attack on secrecy (resp. aliveness, weak agreement), then it admits an attack that involves at most one session of each role. The situation is however slightly more complicated than it may seem at first sight. As we have an infinite number of agent names there is an infinite number of sessions, which one would need to verify separately. Actually we can avoid this combinatorial explosion thanks to the following well-known result [14]: when verifying secrecy properties it is sufficient to consider two agents (an honest agent and a dishonest one). Hence, using this result, we can instantiate 
all the parameters using only two agent names. Similar reduction results also exist for authentication properties (see [14]).

Note that we only consider protocols whose long-term secret keys do not occur in plaintext position. This assumption is required to ensure that the "small scenario", (i.e., the one that involves only $\|\phi\|$ sessions of each role) will violate the same security property $\phi$. We may actually relax this assumption if we consider an execution trace that reveals such a long-term key as a violation of the security property as well. The result is stated in this way in 1 .

Actually, for the security properties presented in the previous section, we can go even further and only consider one honest session of each role.

Corollary 1 . Let $\Pi$ be a $k$-party protocol, $\Pi_{\mathrm{S}}$ (respectively, $\Pi_{\mathrm{A}}, \Pi_{\mathrm{WA}}$ ) be the annotated protocol for modeling secrecy (respectively aliveness and weak agreement) as defined in Section 4.2.1, and $\widetilde{\Pi}_{\mathrm{S}}$ (respectively, $\widetilde{\Pi}_{\mathrm{A}}, \widetilde{\Pi}_{\mathrm{WA}}$ ) the corresponding transformed protocol. Let $T_{0}$ be a set of ground atoms such that $\operatorname{lgKeys}(\Pi) \cap$ plaintext $(\Pi) \subseteq T_{0} \cup \mathcal{K}_{\epsilon}$ and $\overline{\phi_{\mathrm{S}}}$ (respectively $\overline{\phi_{\mathrm{A}}}, \overline{\phi_{\mathrm{WA}}}$ ) an attack formula against secrecy (respectively aliveness and weak agreement) as defined in Section 4.2.1, For $\mathrm{X} \in\{\mathrm{S}, \mathrm{A}, \mathrm{WA}\}$ we have that if $\widetilde{\Pi}_{\mathrm{X}} \models \overline{\phi_{\mathrm{X}}}$ w.r.t. $T_{0}$ then there exists a valid execution trace exec of $\widetilde{\Pi}_{X}$ such that:

$\left\langle\right.$ exec, $\left.T_{0}\right\rangle \models \overline{\phi x}$ and exec involves at most one honest session of each role.

5.3. Alternative ways of tagging protocols. Our transformation is computationally light as it does not add any cryptographic application. However, it increases significantly the size of messages to be encrypted and signed. As an alternative, we may choose to hash the tags. Our results still hold in this setting.

We have also considered an alternative, slightly different transformation that does not include the identities in the tag, i.e., the tag is simply the sequence of nonces. Our main result, Theorem 5.6, still holds as the proof does not use the presence of the identities. However, the stronger results presented on particular properties stated in Corollary 1, do not hold anymore, as the proof crucially relies on the presence of the agent names in the tag. When omitting identities, even for secrecy, we need to additionally check for attacks that involve a session engaged with the attacker. Indeed, on the example of the NeedhamSchroeder protocol the man-in-the-middle attack is not prevented by this weaker tagging scheme. However, the result requires one to also consider one dishonest session for each role, hence including the attack scenario. In both cases, it is important for the tags to be collaborative, i.e. all participants do contribute by adding a fresh nonce. 


\section{— PART II: Proof of our reduction result -}

In this part, we give an overview of the proof of our reduction result stated in Theorem [5.6. Assume that our protocol $\widetilde{\Pi}$ admits an attack.

(1) We first show that there is an attack on a well-formed execution trace (Section 6). In a well-formed execution trace (see Definition 6.4), terms are necessarily tagged with the expected tag, i.e. the tag computed during the initialization phase. Moreover, only names coming from sessions tagged in the same way can be used in the events of those sessions. In order to prove this, we define a transformation - that transforms an execution trace to a well-formed one by abstracting some subterms (those that are not tagged properly using the expected tag) by fresh nonces. We show that this transformation preserves the validity of the trace (Proposition 6.13) as well as the satisfiability of the attack formula under study (Proposition 6.14).

(2) Then, given a set of sessions $S$ and a valid and well-formed execution exec that satisfies the attack formula, we show that exec $\left.\right|_{S}$, i.e. the restriction of exec to the events coming from a session in $S$ is still an execution satisfying the attack formula. Since messages coming from one session can be used to build a message for another session, this can only be achieved by requiring some conditions on $S$. Basically, to ensure the validity of the execution exec $\left.\right|_{S}$, we have to ensure that sessions that share the same tag are either all in $S$ or none of them is in $S$ (see Proposition 7.4). Then, to ensure the satisfiability of the attack formula, we have to keep enough sessions but we can bound a priori the number of sessions that is needed to mount an attack (see Proposition 7.7).

\section{First Step: towards A WELl-FORMED EXECUtion trace}

In this section, we formally define our notion of well-formedness and we propose a transformation that allows us to transform a trace exec into a well-formed one exec (Section 6.2) preserving its validity (Section 6.3) and the satisfiability of the attack formula (Section 6.4).

6.1. Well-formed. The idea behind our notion of well-formedness is to ensure that each term will be properly tagged. Basically, this means that each term has to be tagged with its expected tag, i.e. the one computed during the initialization phase of the protocol (phase 1). From now on, when we consider a trace exec issued from a protocol $\widetilde{\Pi}$, we assume that the events occurring in exec are annotated with their session identifier, and we write exec $=\left[\mathrm{e}_{1}^{\text {sid }}{ }_{1} ; \ldots ; \mathrm{e}_{\ell} \mathrm{e}_{\ell}\right]$ when we want to refer to these annotations explicitly.

The transformation that we consider will abstract some subterms by fresh names from the intruder's knowledge (i.e. names in $\mathcal{N}_{\epsilon}$ ). Those names will be denoted by $n_{t}^{\epsilon, S}$ where $S$ is set of session identifiers, and $t$ is a term. Intuitively, such a name will be used to abstract the subterm $t$ when used in an event from a session sid $\in S$. We assume that those names (which constitute an infinite subset of $\mathcal{N}_{\epsilon}$ ) are not used anywhere else. In particular, they do not occur in the execution trace before applying our transformation.

Definition 6.1 (ExpectedTag(exec, sid)). Let $\Pi$ be a $k$-party protocol and let exec = $\left[\mathrm{e}_{1}^{s i d_{1}} ; \ldots ; \mathrm{e}_{\ell}^{s i d_{\ell}}\right]$ be an execution trace of $\widetilde{\Pi}$. Let sid be a session identifier and $\left[\mathrm{e}_{i_{1}}^{s i d} ; \ldots ; \mathrm{e}_{i_{h}}^{s i d}\right]$ (with $1 \leq i_{1}<\ldots<i_{h} \leq \ell$ ) be the sequence of communication events in exec that are annotated with sid. We define the expected tag of a session sid in exec as 
- ExpectedTag (exec, sid $)=\perp$ when $h<k$,

- ExpectedTag(exec, sid $)=\left\langle m_{1}, \ldots, m_{k}\right\rangle$ otherwise, where for all $j \in\{1, \ldots, k\}, m_{j}$ is such that $\mathrm{e}_{i_{j}}^{\text {sid }}=\operatorname{rcv}\left(m_{j}\right)$ or $\mathrm{e}_{i_{j}}^{\text {sid }}=\operatorname{snd}\left(m_{j}\right)$.

Roughly, the expected tag associated to a session sid is the one obtained by putting together the messages that occur in the $k$ first communication events annotated with sid that occur in exec. When those events do not exist, the expected tag of sid is undefined. We define ExpectedTags(exec) to denote the set of expected tags that occur in the trace exec. More formally, we have that:

$$
\text { ExpectedTags }(\text { exec })=\bigcup_{\text {sid }}\{\text { ExpectedTag }(\text { exec, sid })\} \text {. }
$$

Since a session is the execution of one role, it is likely that several sessions will have the same expected tag. However, note that sessions that correspond to the execution of the same role (e.g. the $j^{\text {th }}$ role) cannot have the same expected tag since the tag will contain a fresh nonce at its $j^{\text {th }}$ position.

Definition 6.2 (sameTagAs(exec, sid)). Let $\Pi$ be a $k$-party protocol and let exec be an execution trace (not necessarily valid) of $\widetilde{\Pi}$. We define sameTagAs(exec, sid) to be the set of sessions sharing the same expected tag with the session sid, i.e.

sameTagAs $($ exec, sid $)=\left\{\begin{array}{l}\{\text { sid }\} \text { if ExpectedTag }(\text { exec, sid })=\perp \\ \left\{\text { sid } \mid \text { ExpectedTag }\left(\text { exec }, \text { sid }^{\prime}\right)=\text { ExpectedTag }(\text { exec }, \text { sid })\right\} \text { otherwise }\end{array}\right.$

Our notion of well-formedness aims to ensure that each event that occurs in a trace is tagged properly. For this, we first define Tags(exec, sid). This set corresponds to the tags that actually occur in the events issued from the session sid in the execution trace exec.

Definition $6.3(\operatorname{Tags}(\mathrm{exec}$, sid $))$. Let $\Pi$ be a $k$-party protocol and exec $=\left[\mathrm{e}_{1}^{s i d_{1}} ; \ldots ; \mathrm{e}_{\ell}^{\text {sid }_{\ell}}\right]$ be an execution trace of $\widetilde{\Pi}$ which is $k$-tagged. Let sid be a session identifier. We define the tags of a session sid in exec as follows:

$$
\text { Tags(exec, sid })=\left\{\left.u\right|_{1.1} \mid u \in \operatorname{CryptSt}\left(\mathrm{e}_{j}^{s i d_{j}}\right) \text { for some } j \in\{1, \ldots, \ell\} \text { such that } \operatorname{sid}_{j}=\text { sid }\right\} \text {. }
$$

We define Tags(exec) to denote the set of tags that occur in the trace exec. More formally, we have that

$$
\operatorname{Tags}(\text { exec })=\bigcup_{\text {sid }} \operatorname{Tags}(\text { exec, sid }) \text {. }
$$

We are now able to define our notion of well-formed execution trace.

Definition 6.4 (well-formed execution trace). Let $\Pi$ be a $k$-party protocol, and exec $=$ $\left[\mathrm{e}_{1}^{s i d_{1}} ; \ldots ; \mathrm{e}_{\ell}^{\text {sid }}\right]$ be an execution trace associated to $\widetilde{\Pi}$. We say that exec is well-formed if:

(1) exec is $k$-tagged, i.e. for all $t \in \mathrm{St}$ (exec), $t$ is $k$-tagged;

(2) Tags (exec, sid $) \subseteq\{$ ExpectedTag (exec, sid $)\}$ for every sid;

(3) For every $i$, we have that names $\left(\mathrm{e}_{i}^{\text {sid }}\right) \subseteq\left\{n_{t}^{\epsilon, S} \mid t \in T\right\} \cup\left\{n_{y}^{\text {sid }} \mid y \in \mathcal{Y}\right.$ and sid $\left.\in S\right\}$ where $S=$ sameTagAs $\left(\right.$ exec, sid $\left._{i}\right)$.

Intuitively, in a well-formed trace, the events of a session sid are $k$-tagged with the expected tag, i.e. the tag defined in the preamble of the session sid. Moreover, the nonces used in a session sid are those that are generated in a session that used the same tag as sid (or they come from the intruder). 
6.2. Our transformation of execution traces. A valid execution trace is not necessarily well-formed. Our goal is to show that we can however always transform an execution trace into a well-formed execution trace. The main idea is to replace each subterm that is not tagged in the expected way with a nonce known by the attacker. The difficulty will be to ensure that the resulting trace is still a valid one (see Section 6.3) and still a witness of the existence of an attack (see Section 6.4).

We first define our transformation on a term. For this we need to introduce the notion of HeadTag

Definition 6.5 (HeadTag $($ exec, $t))$. Let $\Pi$ be a $k$-party protocol and exec be an execution trace (not necessarily valid) of $\widetilde{\Pi}$. We define the head tag of a term $t$ w.r.t. the trace exec, denoted HeadTag (exec, $t)$.

$$
\text { HeadTag }(\text { exec, } t)=\left\{\begin{array}{rr}
\tau & \text { if } t=\mathrm{f}\left(\langle\tau, u\rangle, u_{2}, \ldots, u_{n}\right) \in \text { CryptSt }(t) \\
\perp & \text { and } \tau \in \text { ExpectedTags }(\text { exec })
\end{array}\right.
$$

Roughly, our transformation of a term proceeds as follows. We replace each cryptographic subterm which is not tagged properly with a nonce. We also perform the same kind of replacement on nonces to ensure that sessions that are tagged differently will not share any nonces.

Definition $6.6\left(\bar{t}^{\text {exec,sid }}\right)$. Let $\Pi$ be a $k$-party protocol, exec be an execution trace (not necessarily valid) of $\widetilde{\Pi}$, sid be a session identifier and $\tau=$ ExpectedTag(exec, sid).

- $\bar{n}^{\mathrm{exec}, s i d}=n_{n}^{\epsilon, S}$ if $n \in \mathcal{N}^{\epsilon}$ or if $\tau=\perp$, where $S=$ sameTagAs(exec, sid);

- $\overline{n_{y}^{s i d^{\prime}}}$ exec,sid $=\left\{\begin{array}{l}n_{y}^{s i d^{\prime}} \text { if } \text { sid }^{\prime} \in \text { sameTagAs(exec, sid) } \\ n_{n_{y}^{\text {sid }}}^{\epsilon, S} \text { where } S=\text { sameTagAs(exec, sid) otherwise }\end{array}\right.$

- $\bar{a}^{\text {exec,sid }}=a$ if $a$ is the name of an agent;

- $\overline{\mathrm{f}\left(a_{1}, \ldots, a_{n}\right)}{ }^{\text {exec, sid }}=\mathrm{f}\left(a_{1}, \ldots, a_{n}\right)$ for $\mathrm{f} \in\{$ shk, pub, priv $\}$

- $\overline{\langle u, v\rangle}^{\mathrm{exec}, s i d}=\left\langle\bar{u}^{\mathrm{exec}, \text { sid }}, \bar{v}^{\mathrm{exec}, \text { sid }}\right\rangle$;

- $\overline{\mathrm{f}\left(u_{1}, \ldots, u_{n}\right)}{ }^{\text {exec }, \text { sid }}=\left\{\begin{array}{r}\mathrm{f}\left(\overline{u_{1}}{ }^{\text {exec }, \text { sid }}, \ldots, \overline{u_{n}} \text { exec, sid }\right) \\ \text { if HeadTag }\left(\text { exec, } \mathrm{f}\left(u_{1}, \ldots, u_{n}\right)\right)=\tau \text { and } \tau \neq \perp \\ \left.n_{\mathrm{f}\left(u_{1}, \ldots, u_{n}\right)}^{\epsilon} \text { where } S=\text { sameTagAs(exec, sid }\right) \text { otherwise }\end{array}\right.$

for any $\mathrm{f} \in\{$ encs, enca, sign, $\mathrm{h}\}$.

We extend our transformation on a trace in the expected way.

Definition $6.7(\overline{\mathrm{exec}})$. Let $\Pi$ be a protocol and exec $=\left[\mathrm{e}_{1}^{s i d_{1}} ; \ldots ; \mathrm{e}_{\ell}^{\text {sid }}{ }_{\ell}\right]$ an execution trace (not necessarily valid) of $\widetilde{\Pi}$. We define $\overline{\mathrm{exec}}={\overline{\mathrm{e}_{1}}}^{\mathrm{exec}, \text { sid }_{1}} ; \ldots ; \overline{\mathrm{e}}_{\ell}{ }^{\mathrm{exec}, \text { sid }} \ell$, where

$$
\overline{\mathrm{e}}^{\mathrm{exec}, \text { sid }}= \begin{cases}\mathrm{P}\left({\overline{u_{1}}}^{\mathrm{exec}, \text { sid }}, \ldots,{\overline{u_{n}}}^{\mathrm{exec}, \text { sid }}\right) & \text { if } \mathrm{e}=\mathrm{P}\left(u_{1}, \ldots, u_{n}\right) \\ \operatorname{snd}\left(\bar{u}^{\mathrm{exec}, \text { sid }}\right) & \text { if } \mathrm{e}=\operatorname{snd}(u) \\ \operatorname{rcv}\left(\bar{u}^{\mathrm{exec}, \text { sid }}\right) & \text { if } \mathrm{e}=\operatorname{rcv}(u)\end{cases}
$$

With this transformation, we still get a trace associated to the protocol under study. Moreover, the resulting execution trace is well-formed. This is formally proved in Appen$\operatorname{dix}$ A (Lemma A.1 and Lemma A.2). 
Proposition 6.8. Let $\Pi$ be a k-party protocol, and exec be an execution trace associated to $\widetilde{\Pi}$ (not necessarily a valid one). We have that $\overline{\mathrm{exec}}$ is a well-formed execution trace (not necessarily a valid one) associated to the protocol $\widetilde{\Pi}$.

6.3. Validity. Now, we show that the resulting execution trace, i.e. the one obtained by applying our transformation - , is still a valid one. In particular, we have to show that each term that occurs in a receive event is deducible from the initial knowledge of the attacker and the messages that have been sent so far. For this, we rely on the notion of simple proofs previously introduced in [17.

Definition 6.9 (simple proof). Let $T_{1} \subseteq T_{2} \subseteq \cdots \subseteq T_{n}$. We say that a proof $\pi$ of $T_{i} \vdash u$ is left-minimal if, whenever there is a proof of $T_{j} \vdash u$ for some $j<i$, then $\pi$ is also a proof of $T_{j} \vdash u$. Then, we say that a proof $\pi$ is simple if

(1) any subproof of $\pi$ is left-minimal,

(2) a composition rule of the form $\frac{u_{1} \quad u_{2}}{u}$ is never followed by a decomposition rule leading to $u_{1}$ or $u_{2}$, and

(3) any term of the form $\left\langle u_{1}, u_{2}\right\rangle$ obtained by application of a decomposition rule or labelling a leaf is directly followed by a projection rule.

Example 6.10. Let $T_{1}=\left\{n_{1}\right\}$ and $T_{2}=\left\{n_{1}\right.$, encs $\left.\left(\left\langle n_{1}, n_{2}\right\rangle, k\right), k\right\}$. We have $T_{2} \vdash\left\langle n_{1}, n_{2}\right\rangle$ with the proof tree $\pi$ described below. However, $\pi$ is not a simple proof of $T_{2} \vdash\left\langle n_{1}, n_{2}\right\rangle$. Indeed, the term $\left\langle n_{1}, n_{2}\right\rangle$ has been obtained by an application of a decomposition rule. Thus, by Condition (31) of Definition 6.9 we have to decompose it. A simple proof of $T_{2} \vdash\left\langle n_{1}, n_{2}\right\rangle$ is the proof tree $\pi^{\prime}$ described below.

$$
\pi=\left\{\frac{\operatorname{encs}\left(\left\langle n_{1}, n_{2}\right\rangle, k\right) \quad k}{\left\langle n_{1}, n_{2}\right\rangle} \quad \pi^{\prime}=\left\{\begin{array}{l}
\frac{\operatorname{encs}\left(\left\langle n_{1}, n_{2}\right\rangle, k\right) k}{\left\langle n_{1}, n_{2}\right\rangle} \\
\frac{n_{1}}{n_{2}}
\end{array}\right.\right.
$$

As it was done in [17] in a slightly different setting, we can show that it is always possible to consider such a proof tree, i.e. if there is a proof of $T_{i} \vdash u$, then there is a simple proof of it (w.r.t. a sequence $T_{1} \subseteq T_{2} \subseteq \cdots \subseteq T_{n}$ ). Given a simple proof $\pi$ of $T_{i} \vdash u$, we can also show a locality lemma (by structural induction on $\pi$ ) allowing us to characterize the terms that occur in such a proof tree.

Lemma 6.11 (locality). Let $T_{1} \subseteq T_{2} \subseteq \cdots \subseteq T_{n}$ be a set of terms and $u$ be a term such that $T_{i} \vdash u$. Let $\pi$ be a simple proof of $T_{i} \vdash u$. We have that $\pi$ only involves terms in $\operatorname{St}\left(T_{i} \cup\{u\}\right) \cup \mathcal{K}_{\epsilon} \cup \mathcal{N}_{\epsilon} \cup \mathcal{A} \cup\{\operatorname{pub}(a) \mid a \in \mathcal{A}\}$. Moreover, if $\pi$ ends with an instance of a decomposition rule (or is reduced to a leaf), we have that $\pi$ only involves terms in $\mathrm{St}\left(T_{i}\right) \cup \mathcal{K}_{\epsilon} \cup \mathcal{N}_{\epsilon} \cup \mathcal{A} \cup\{\operatorname{pub}(a) \mid a \in \mathcal{A}\}$.

Now, relying on this notion of simple proof, we can show that deducibility is preserved by our transformation. This is the key lemma to ensure the validity of the resulting trace.

Lemma 6.12. Let $\Pi$ be a k-party protocol and exec $=\left[\mathrm{e}_{1}^{\text {sid }}{ }_{1} ; \ldots ; \mathrm{e}_{\ell}^{\text {sid }}\right]_{\ell}$ be a valid execution trace of $\widetilde{\Pi}, w . r . t$. some set $T_{0}$ of ground atoms. Let $i \in\{0, \ldots, \ell\}$ and $t$ be a term such that $\mathrm{K}\left(\operatorname{exec}_{i}\right) \cup T_{0} \vdash t$. We have that $\mathrm{K}\left(\overline{\operatorname{exec}}_{i}\right) \cup T_{0} \vdash \bar{t}^{\mathrm{exec}, \text { sid }}$ for any sid. 
Proof. (sketch) Let $\left.\operatorname{tr}=\left[\mathrm{ee}_{1}^{s i d_{1}} ; \ldots ; \mathrm{ee}_{\ell}^{\text {sid }}\right]_{\ell}\right]$ be the symbolic trace associated to exec and $\sigma$ be the substitution such that $\operatorname{dom}(\sigma)=\operatorname{vars}(\operatorname{tr})$ and exec $=\operatorname{tr} \sigma$. Let $i \in\{0, \ldots, \ell\}$. Let $\pi$ be a simple proof of $\mathrm{K}\left(\operatorname{exec}_{i}\right) \cup T_{0} \vdash t$. We prove that $\mathrm{K}\left(\overline{\operatorname{exec}}_{i}\right) \cup T_{0} \vdash \bar{t}^{\text {exec, sid }}$ by induction on $(i, \pi)$. If $i=0$ and $\pi$ is a simple proof reduced to a leaf (possibly followed by some projection rules), then we have that $T_{0} \vdash t$, and $\pi$ is necessarily reduced to a leaf since $T_{0}$ only contains atomic terms. Let sid be a session identifier, we have that $\bar{t}^{\text {exec,sid }} \in\{t\} \cup \mathcal{N}_{\epsilon}$ since $t$ is an atomic term. This allows us to conclude that $T_{0} \vdash \bar{t}^{\text {exec,sid }}$. Now, we distinguish two cases depending on the last rule of $\pi$.

- The proof $\pi$ ends with an instance of a composition rule, i.e. $t=\mathrm{f}\left(t_{1}, \ldots, t_{n}\right)$ for some $\mathrm{f} \in\{\langle$,$\rangle , encs, enca, sign, \mathrm{h}\}$ and some terms $t_{1}, \ldots, t_{n}$.

According to Definition [6.6, we have that $\bar{t}^{\text {exec,sid }} \in \mathcal{N}^{\epsilon} \cup\left\{\mathrm{f}\left({\overline{t_{1}}}^{\text {exec,sid }}, \ldots,{\overline{t_{n}}}^{\text {exec,sid }}\right)\right\}$. If $\bar{t}^{\text {exec,sid }} \in \mathcal{N}_{\epsilon}$, we easily conclude that $\mathrm{K}\left(\overline{\operatorname{exec}}_{i}\right) \cup T_{0} \vdash \bar{t}^{\text {exec, sid }}$. Otherwise, since $\pi$ ends with a composition rule, we have that $\mathrm{K}\left(\operatorname{exec}_{i}\right) \cup T_{0} \vdash t_{1}, \ldots, \mathrm{K}\left(\operatorname{exec}_{i}\right) \cup T_{0} \vdash t_{n}$. Moreover, the simple proofs witnessing these facts are strict subproofs of $\pi$ that are also simple. Hence, we can apply our induction hypothesis and conclude that $\mathrm{K}\left(\overline{\operatorname{exec}}_{i}\right) \cup T_{0} \vdash$ $\mathrm{f}\left({\overline{t_{1}}}^{\text {exec }, \text { sid }}, \ldots,{\overline{t_{n}}}^{\text {exec }, \text { sid }}\right)$.

- The proof ends with the application of a decomposition rule (but not a projection) possibly followed by several applications of the projection rules until the resulting term is not a pair.

We will here present the case of the symmetric decryption rule, but all the other decomposition rules (including the case of a proof reduced to a leaf) can be handled in a similar way. For some terms $t_{1}$ and $t_{2}$, the proof $\pi$ is of the form

$\frac{\frac{\vdots}{\mathrm{K}\left(\operatorname{exec}_{i}\right) \cup T_{0} \vdash \operatorname{encs}\left(t_{1}, t_{2}\right)} \quad \frac{\vdots}{\frac{\mathrm{K}\left(\operatorname{exec}_{i}\right) \cup T_{0} \vdash t_{1}}{\mathrm{~K}\left(\operatorname{exec}_{i}\right) \cup T_{0} \vdash t_{2}}}}{\frac{\vdots}{\mathrm{K}\left(\operatorname{exec}_{i}\right) \cup T_{0} \vdash t}}$

Let us first note that, by locality (Lemma 6.11) of $\pi$ we know that encs $\left(t_{1}, t_{2}\right) \in$ $\operatorname{St}\left(\mathrm{K}\left(\operatorname{exec}_{i}\right)\right) \cup T_{0} \cup \mathcal{K}_{\epsilon} \cup \mathcal{N}_{\epsilon} \cup\{\operatorname{pub}(a) \mid a \in \mathcal{A}\}$, and by atomicity of $T_{0}, \mathcal{N}_{\epsilon}, \mathcal{K}_{\epsilon}$ and $\{\operatorname{pub}(a) \mid a \in \mathcal{A}\}$, we know that encs $\left(t_{1}, t_{2}\right) \in \mathrm{St}\left(\mathrm{K}\left(\operatorname{exec}_{i}\right)\right)$. (In case of a proof reduced to a leaf, and if there is no projection rule, we may have that $t \in T_{0}$. In such a case, as in the base case, we have that $T_{0} \vdash \bar{t}^{\text {exec,sid }}$ and we easily conclude.) Hence, there exists $k \leq i$ such that $\mathrm{e}_{k}^{s i d_{k}}=\operatorname{snd}(u)$ and encs $\left(t_{1}, t_{2}\right) \in \mathrm{St}(u)$. Let $k_{0}$ be the smallest such $k$ and $u_{0}, u_{0}^{\prime}$ be such that $\mathrm{e}_{k_{0}}^{s i d_{k_{0}}}=\operatorname{snd}\left(u_{0}\right)$ and $\mathrm{ee}_{k_{0}}^{\operatorname{sid}_{k_{0}}}=\operatorname{snd}\left(u_{0}^{\prime}\right)$. Hence, we have that $u_{0}=u_{0}^{\prime} \sigma$. In order to prove the result, we first establish the following claim (proved in Appendix C). Claim: We have that $\overline{\operatorname{encs}\left(t_{1}, t_{2}\right)}{ }^{\text {exec, } s i d_{k_{0}}}=\operatorname{encs}\left({\overline{t_{1}}}^{\text {exec, } s i d_{k_{0}}},{\overline{t_{2}}}^{\text {exec, sid }}{ }_{k_{0}}\right)$. Now, relying on this claim and applying the induction hypothesis, we have that:

$-\mathrm{K}\left(\overline{\mathrm{exec}}_{i}\right) \cup T_{0} \vdash \operatorname{encs}\left({\overline{t_{1}}}^{\text {exec }, s i d_{k_{0}}},{\overline{t_{2}}}^{\text {exec }, s i d_{k_{0}}}\right)$; and

$-\mathrm{K}\left(\overline{\operatorname{exec}}_{i}\right) \cup T_{0} \vdash{\overline{t_{2}}}^{\mathrm{exec}, s_{i} k_{0}}$. 
This allows us to deduce that $\mathrm{K}\left(\overline{\mathrm{exec}}_{i}\right) \cup T_{0} \vdash{\overline{t_{1}}}^{\mathrm{exec}, s i d_{k_{0}}}$. In order to establish that $\mathrm{K}\left(\overline{\operatorname{exec}}_{i}\right) \cup T_{0} \vdash \bar{t}^{\text {exec,sid }}$, we need to distinguish two cases:

Case 1. $t \in \mathcal{A}, t=\operatorname{pub}(a)$ or $t=\mathrm{f}\left(a_{1}, \ldots, a_{n}\right)$ for some $\mathrm{f} \in\{$ shk, priv $\}$. In such a case, we have that $\bar{t}^{\text {exec,sid }}=\bar{t}^{\text {exec,sid } k_{0}}=t$. Hence, we have that $\mathrm{K}\left(\overline{\operatorname{exec}}_{i}\right) \cup T_{0} \vdash \bar{t}^{\text {exec,sid }}$ by applying some projection rules on the proof of $\mathrm{K}\left(\overline{\operatorname{exec}}_{i}\right) \cup T_{0} \vdash \bar{t}_{1}{ }^{\mathrm{exec}, s_{i d} k_{0}}$.

Case 2. $t \in \mathcal{N}$ or $t=\mathrm{f}\left(t_{1}^{\prime}, \ldots, t_{m}^{\prime}\right)$ for some $\mathrm{f} \in\{$ encs, enca, $\mathrm{h}, \mathrm{sign}\}$. First, if $\bar{t}^{\text {exec, sid }}$ can be

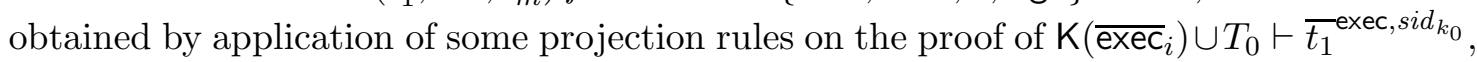
then we easily conclude. Otherwise, it means that the term $t$ is not abstracted in the same way in both cases. In such a case, we have that either $\bar{t}^{\text {exec,sid }} \in \mathcal{N}_{\epsilon}$ or $\bar{t}^{\text {exec }, s i d_{k_{0}}} \in \mathcal{N}_{\epsilon}$. In the first case, we easily conclude. In the second case, i.e. $\bar{t}^{\text {exec,sid } k_{0}} \in \mathcal{N}_{\epsilon}$ but $\bar{t}^{\text {exec,sid }} \notin \mathcal{N}_{\epsilon}$, we can show that $t$ is a subterm of $u_{0}$ that either occurs as a component of $u_{0}$ or in the term $x \sigma$ for some $x \in \operatorname{vars}\left(u_{0}^{\prime}\right)$. Actually, the first case is not possible since we have assumed that $\bar{t}^{\text {exec,sid }}$ k $_{0} \in \mathcal{N}_{\epsilon}$. Thus, only the second case remains. Thanks to the origination property, we know that $t$ will occur in a previous receive event and we will be able to show that $t$ was deducible using a smaller prefix of the trace allowing us to conclude by applying our induction hypothesis.

Since our transformation preserves the deducibility relation, we can now prove the validity of the resulting trace by induction on the length of the original trace.

Proposition 6.13. Let $\Pi$ be a k-party protocol and exec $=\left[\mathrm{e}_{1}^{s i d_{1}} ; \ldots ; \mathrm{e}_{\ell}^{s i d_{\ell}}\right]$ be a valid execution trace associated to $\widetilde{\Pi}$, w.r.t. some initial intruder knowledge $T_{0}$. We have that $\overline{\mathrm{exec}}$ is a well-formed and valid execution trace associated to $\widetilde{\Pi}$ w.r.t $T_{0}$.

Proof. First, according to Proposition 6.8 , we know that $\overline{\text { exec }}$ is an execution trace associated to $\widetilde{\Pi}$ which is well-formed. It remains to establish its validity w.r.t. $T_{0}$. We show by induction on $i$ that for all $i \in\{1, \ldots, \ell\},(\overline{\mathrm{exec}})_{i}$ is a valid execution trace. The base case, i.e. the empty trace $(\overline{\mathrm{exec}})_{i}=[]$, is trivially valid. For the inductive step, we assume that $(\overline{\mathrm{exec}})_{\ell-1}$ is valid and we have to establish the validity of $\overline{\text { exec }}=\overline{\operatorname{exec}} \ell_{\ell}$. We distinguish 2 cases according to the nature of the last event in the trace.

Case $\mathrm{e}_{\ell}^{\text {sid } d_{\ell}}=\mathrm{P}\left(t_{1}, \ldots, t_{n}\right)$ or $\mathrm{e}_{\ell}^{\text {sid }}=\operatorname{snd}(t)$. By induction hypothesis, we know that $(\overline{\mathrm{exec}})_{\ell-1}$ is a valid execution trace, and this is enough to conclude to the validity of $\overline{\text { exec. }}$

Case $\mathrm{e}_{\ell}^{\text {sid }}=\operatorname{rcv}(t)$. By induction hypothesis, we know that $(\overline{\mathrm{exec}})_{\ell-1}$ is a valid execution trace. To conclude to the validity of $\overline{\text { exec }}$, we only need to establish that $\mathrm{K}\left((\overline{\mathrm{exec}})_{\ell-1}\right) \cup T_{0} \vdash$ $\bar{t}^{\mathrm{exec}, \text { sid }_{\ell}}$. Since we know that exec is a valid execution trace, we have that $\mathrm{K}\left(\mathrm{exec}_{\ell-1}\right) \cup T_{0} \vdash t$. Applying Lemma 6.12, we conclude that $\mathrm{K}\left((\overline{\mathrm{exec}})_{\ell-1}\right) \cup T_{0} \vdash \bar{t}^{\mathrm{exec}, \text { sid }_{\ell}}$. This allows us to deduce that $\overline{\text { exec }}$ is valid.

6.4. Satisfiability. The goal of this section is to show that the trace $\overline{\text { exec }}$ resulting of the application of our transformation will still satisfy the attack formula $\exists x_{1} \ldots . \exists x_{n} . \phi$ under study. To show the validity of such a formula on the trace $\overline{\text { exec, }}$, we have to exhibit a substitution $\sigma^{\prime}$ for which $\left\langle\overline{\mathrm{exec}}, T_{0}\right\rangle \models \phi \sigma^{\prime}$. By hypothesis, we know that $\left\langle\right.$ exec, $\left.T_{0}\right\rangle \models \phi \sigma$ for some $\sigma$. Thus, the idea is to consider the substitution $\sigma^{\prime}=\left\{x_{1} \mapsto \overline{x_{1} \sigma^{\text {exec }, \text { sid }}}{ }, \ldots, x_{n} \mapsto \overline{x_{n} \sigma^{\text {exec }, s i d_{n}}}\right\}$ where $\operatorname{sid}_{1}, \ldots$, sid $_{n}$ correspond to the sessions from which the terms $x_{1} \sigma, \ldots, x_{n} \sigma$ come from. 
Proposition 6.14. Let $\Pi$ be a protocol, exec be an execution trace of $\widetilde{\Pi}$ w.r.t. some initial intruder knowledge $T_{0}$, and $\phi$ be an attack formula. We have that

$$
\left\langle\text { exec, } T_{0}\right\rangle \models \phi \Rightarrow\left\langle\overline{\mathrm{exec}}, T_{0}\right\rangle \models \phi .
$$

The proof is done by structural induction on the formula and its details can be found in Appendix D. The technically difficult part is to formally link each variable existentially quantified in $\phi$ with the term it has been substituted with in order to satisfy the formula.

\section{SECONd STEP: REDUCING THE NUMBer OF SESSIONS}

Now, our goal is to reduce the number of sessions that are involved in an execution trace witnessing the existence of an attack in order to match the bound announced in Theorem 5.6. the attack trace has to involved at most $\|\phi\|$ sessions of each role. The idea will be to identify a set of sessions $S$ and to remove all the events that do not originate from a session in $S$ according to the formal definition stated below.

Definition 7.1 (restriction of $\operatorname{tr}$ to $S$ ). Let $\Pi$ be a protocol, exec $\left.=\left[\mathrm{e}_{1}^{s i d_{1}} ; \ldots ; \mathrm{e}_{\ell}^{\text {sid }}\right]_{\ell}\right]$ be an execution of $\Pi$, w.r.t. some set $T_{0}$ of ground atoms, and $S$ be a set of session identifiers. The restriction of exec to $S$ is defined as the trace exec| $\left.\right|_{S}=\left[\mathrm{e}_{i_{1}}\right.$ sid $_{i_{1}} ; \ldots ; \mathrm{e}_{i_{h}}$ sid $\left._{i_{h}}\right]$ satisfying the following: $i_{1}<\ldots<i_{h}$ and for all $j \in\{1, \ldots, \ell\}$, there exists $k \in\{1, \ldots, h\}$ such that $j=i_{k}$ if and only if $\operatorname{sid}_{j} \in S$.

Given a valid and well-formed execution exec and a set of sessions $S$, the goal of this section is to show that the restriction exec $\left.\right|_{S}$ is a valid and well-formed execution. Since messages coming from one session can be used to build a message for another session, to prove such a result, it is important to require some conditions on $S$. Basically, we will consider a set $S$ that satisfies the following requirement:

for all $\operatorname{sid}_{1}$ and $\operatorname{sid}_{2}$ such that sameTagAs(exec, $\left.\operatorname{sid}_{1}\right)=$ sameTagAs(exec, $\left.\operatorname{sid}_{2}\right)$, we have that $\operatorname{sid}_{1} \in S$ if and only if $\operatorname{sid}_{2} \in S$.

This means that sessions using the same tag should have the same status w.r.t. the set $S$.

In the following of this section we will first show that

(i) such a restricted execution is still a valid execution, and

(ii) that the restriction preserves satisfiability of attack formulas.

7.1. Validity of the restriction. First, we show that in a well-formed and valid execution trace, terms that occur in sessions that are tagged differently do not share any name.

Lemma 7.2. Let $\Pi$ be a k-party protocol, and $\mathrm{exec}=\left[\mathrm{e}_{1}^{\text {sid }} ; \ldots ; \mathrm{e}_{\ell}^{\text {sid }}\right]_{\ell}$ be a well-formed valid execution of $\widetilde{\Pi}$ w.r.t. some set $T_{0}$ of ground atoms. Let sess 1 and sess $s_{2}$ be two session identifiers. We have that:

$$
\begin{aligned}
& \text { sameTagAs } \left.\left(\text { exec, } \text { sess }_{1}\right) \neq \text { sameTagAs(exec, } \text { sess }_{2}\right) \\
& \text { implies } \\
& \text { names }\left(\text { exec, sess }{ }_{1}\right) \cap \text { names }(\text { exec, sess } 2)=\emptyset
\end{aligned}
$$

where names (exec, sess $)=\left\{u \mid u \in \operatorname{names}\left(\mathrm{e}_{j}^{\text {sid }_{j}}\right)\right.$ for some $1 \leq j \leq \ell$ such that sid $_{j}=$ sess $\}$. 
The goal of the next lemma is to show that deducibility is preserved when we consider the trace exec $\left.\right|_{S}$. Note that the previous lemma allows us to ensure that the terms we removed from the trace are "sufficiently disjoint" from the ones we keep. This is important to ensure that deducibility is preserved in the trace exec $\left.\right|_{S}$.

Lemma 7.3. Let $\Pi$ be a k-party protocol, and exec $=\left[\mathrm{e}_{1}^{s i d_{1}} ; \ldots ; \mathrm{e}_{\ell}^{\text {sid }_{\ell}}\right]$ a well-formed valid execution of $\widetilde{\Pi}$ w.r.t. some set $T_{0}$ of ground atoms, and such that $T_{0} \cup \mathrm{K}(\mathrm{exec}) \forall k$ for any $k \in$ lgKeys $\backslash\left(\mathcal{K}_{\epsilon} \cup T_{0}\right)$ (exec does not reveal any long term keys). Let $S$ be a set of sessions such that:

for all session identifiers sess ${ }_{1}$ and sess $s_{2}$ such that sameTagAs(exec, sess 1$)=$ sameTagAs(exec, sess $\left.s_{2}\right)$, we have that sess $s_{1} \in S$ if and only if sess $_{2} \in S$.

For all term $t \in \mathrm{St}\left(\left.\mathrm{exec}\right|_{S}\right)$ such that $T_{0} \cup \mathrm{K}(\mathrm{exec}) \vdash t$, we have that $T_{0} \cup \mathrm{K}\left(\left.\operatorname{exec}\right|_{S}\right) \vdash t$.

Now, relying on Lemma 7.3, we are able to show that the trace exec $\left.\right|_{S}$ is valid.

Proposition 7.4. Let $\Pi$ be a k-party protocol, and exec $=\left[\mathrm{e}_{1}^{s i d_{1}} ; \ldots ; \mathrm{e}_{\ell}^{\text {sid }}\right]$ a well-formed valid execution of $\widetilde{\Pi}$ w.r.t. some set $T_{0}$ of ground atoms, and such that $T_{0} \cup \mathrm{K}(\mathrm{exec}) \forall k$ for any $k \in$ lgKeys $\backslash\left(\mathcal{K}_{\epsilon} \cup T_{0}\right.$ ) (exec does not reveal any long term keys). Let $S$ be a set of sessions such that:

for all session identifiers sess 1 and sess $s_{2}$ such that sameTagAs $\left(\mathrm{exec}\right.$, sess $\left._{1}\right)=$ sameTagAs(exec, sess 2$)$, we have that sess ${ }_{1} \in S$ if and only if sess $_{2} \in S$.

We have that exec| $\left.\right|_{S}$ is also a well-formed and valid execution of $\widetilde{\Pi}$ w.r.t. $T_{0}$.

Proof. Let $1 \leq i_{1}<\cdots<i_{n} \leq \ell$ such that exec $\left.\right|_{S}=\left[\mathrm{e}_{i_{1}}^{s i d_{i_{1}}} ; \ldots ; \mathrm{e}_{i_{n}}^{s i d_{i_{n}}}\right]$. We prove by induction on the length $n$ of exec $\left.\right|_{S}$, that exec $\left.\right|_{S}$ is a valid execution of $\widetilde{\Pi}$, w.r.t $T_{0}$.

Base case: If $n=0$ we have that $\left.\operatorname{exec}\right|_{S}=[]$, and thus exec $\left.\right|_{S}$ is a valid execution of $\widetilde{\Pi}$ w.r.t. $T_{0}$.

Inductive case: By induction hypothesis, we know that

$$
\left[\mathrm{e}_{i_{1}}^{s i d_{i_{1}}} ; \ldots ; \mathrm{e}_{i_{n-1}}^{s i d_{i_{n-1}}}\right]
$$

is a valid execution of $\widetilde{\Pi}$ w.r.t. $T_{0}$. If $e_{n}^{s i d_{n}}$ is a send or a status event, then

$$
\left.\operatorname{exec}\right|_{S}=\left[\mathrm{e}_{i_{1}}^{\text {sid }_{i_{1}}} ; \ldots ; \mathrm{e}_{i_{n-1}}^{\text {sid }_{i_{n-1}}} ; \mathrm{e}_{i_{n}}^{s i d_{i_{n}}}\right]
$$

is a valid execution of $\widetilde{\Pi}$ w.r.t. $T_{0}$ (see Definition 3.8). On the other hand, if $\mathrm{e}_{n}^{s i d_{n}}$ is a receive event, i.e. $\mathrm{e}_{n}^{s i d_{n}}=\operatorname{rcv}(t)$, we need to show

$$
T_{0} \cup \mathrm{K}\left(\left[\mathrm{e}_{i_{1}}^{s i d_{i_{1}}} ; \ldots ; \mathrm{e}_{i_{n-1}}^{s i d_{i_{n-1}}}\right]\right) \vdash t
$$

knowing that

$$
T_{0} \cup \mathrm{K}\left(\left[\mathrm{e}_{1}^{\operatorname{sid}_{1}} ; \ldots ; \mathrm{e}_{\left(i_{n}\right)-1}^{s i d_{\left(i_{n}\right)-1}}\right]\right) \vdash t
$$

which because $e_{i_{n}}$ is a reception event implies that $T_{0} \cup \mathrm{K}(\mathrm{exec}) \vdash t$. But then, according to Lemma 7.3 we know that $T_{0} \cup \mathrm{K}\left(\left.\operatorname{exec}\right|_{S}\right) \vdash t$. It suffices now to notice that by definition of $\mathrm{K}()$, because $e_{i_{n}}$ is a reception event, we have that:

$$
\mathrm{K}\left(\left[\mathrm{e}_{i_{1}}^{s i d_{i_{1}}} ; \ldots ; \mathrm{e}_{i_{n-1}}^{s i d_{i_{n-1}}}\right]\right)=\mathrm{K}\left(\left[\mathrm{e}_{i_{1}}^{s i d_{i_{1}}} ; \ldots ; \mathrm{e}_{i_{n}}^{s i d_{i_{n}}}\right]\right)=\mathrm{K}\left(\left.\mathrm{exec}\right|_{S}\right) .
$$


This concludes the proof that exec $\left.\right|_{S}$ is a valid execution of $\widetilde{\Pi}$ w.r.t. the initial intruder knowledge $T_{0}$. Finally, it is obvious that exec $\left.\right|_{S}$ satisfies the 3 conditions of well-formedness (Definition 6.4), from the hypothesis that exec does.

7.2. Satisfiability of the formula. The way the set $S$ of sessions is chosen depends on the sessions that are needed to satisfy the attack formula under study. We therefore introduce the notion of witness sessions which for a given formula $\phi$ can be used to witness that $\phi$ holds.

Definition 7.5 (witness sessions, Ws). Let $\Pi$ be a protocol, $\phi$ a closed quantifier-free formula of $\mathcal{L}$, and $T_{0}$ be a set of ground atoms. Let exec $=\left[\mathrm{e}_{1}^{s i d_{1}} ; \ldots ; \mathrm{e}_{\ell}^{s i d_{\ell}}\right]$ be a valid execution of $\Pi$ (w.r.t. $T_{0}$ ) satisfying $\phi$, i.e. $\left\langle\right.$ exec, $\left.T_{0}\right\rangle \models \phi$. We define the set of sessions Ws $($ exec, $\phi)$ witnessing that $\left\langle\right.$ exec, $\left.T_{0}\right\rangle \models \phi$ by structural induction on $\phi$ as follows:

- Ws $($ exec, $\neg \phi)=\mathrm{Ws}^{-}($exec, $\phi)$;

- Ws $($ exec, true $)=W s($ exec, learn $(t))=W s($ exec, $C(t))=\emptyset$;

- $\mathrm{Ws}\left(\mathrm{Q}\left(t_{1}, \ldots, t_{n}\right)\right)=\left\{\operatorname{sid}_{\ell}\right\}$;

- Ws $(\operatorname{exec}, \diamond \phi)=\mathrm{Ws}\left(\operatorname{exec}_{i}, \phi\right)$ where $i$ is such that $\left\langle\operatorname{exec}_{i}, T_{0}\right\rangle \models \phi$;

- Ws $\left(\right.$ exec, $\left.\phi_{1} \vee \phi_{2}\right)=\mathrm{Ws}\left(\right.$ exec, $\left.\phi_{1}\right)$ if $\left\langle\right.$ exec, $\left.T_{0}\right\rangle \models \phi_{1}$ and $\mathrm{Ws}\left(\right.$ exec, $\left.\phi_{2}\right)$ otherwise;

where

- $\mathrm{Ws}^{-}($exec, $\neg \phi)=\mathrm{Ws}($ exec, $\phi)$;

- $\mathrm{Ws}^{-}($exec, true $)=\mathrm{Ws}^{-}($exec, learn $(t))=\mathrm{Ws}^{-}($exec, $\mathrm{C}(t))=\mathrm{Ws}^{-}($exec, $\diamond \phi)=\emptyset$;

- $\mathrm{Ws}^{-}\left(\mathrm{Q}\left(t_{1}, \ldots, t_{n}\right)\right)=\left\{\operatorname{sid}_{\ell}\right\}$ when length $(\mathrm{exec})>0$ and $\emptyset$ otherwise;

- $\mathrm{Ws}^{-}\left(\right.$exec, $\left.\phi_{1} \vee \phi_{2}\right)=\mathrm{Ws}^{-}\left(\right.$exec, $\left.\phi_{1}\right) \cup \mathrm{Ws}^{-}\left(\right.$exec, $\left.\phi_{2}\right)$.

Intuitively, we keep in the trace the sessions that are needed to satisfy the formula under study. Essentially, we have to keep those that are used to satisfy the status events occurring in the formula.

Lemma 7.6. Let $\Pi$ be a k-party protocol, and exec $\left.=\left[\mathrm{e}_{1}^{\text {sid } d_{1}} ; \ldots ; \mathrm{e}_{\ell}^{\text {sid }}\right]_{\ell}\right]$ be a valid and wellformed execution of $\widetilde{\Pi}$ w.r.t. some set $T_{0}$ of ground atoms such that $T_{0} \cup \mathrm{K}(\mathrm{exec}) \forall k$ for any $k \in$ lgKeys $\backslash\left(\mathcal{K}_{\epsilon} \cup T_{0}\right)$. Let $\phi=\exists x_{1} \ldots \exists x_{n} . \psi$ be an attack formula of $\mathcal{L}$, and $\sigma$ be a ground substitution such that $\left\langle\right.$ exec, $\left.T_{0}\right\rangle \models \psi \sigma$. Let $S$ be a set of session identifiers such that:

(1) Ws $($ exec, $\psi \sigma) \subseteq S$, and

(2) $\forall$ sess $_{1}$, sess $_{2}$ with ExpectedTag $($ exec, sess 1$)=$ ExpectedTag $($ exec, sess 2$)$, we have that sess $_{1} \in S$ if and only if sess $s_{2} \in S$.

We have that exec $\left.\right|_{S}$ is an execution of $\widetilde{\Pi}$ that satisfies $\phi$, i.e. $\left\langle\left.\operatorname{exec}\right|_{S}, T_{0}\right\rangle \models \phi$.

Proof. (sketch) The idea is to show that $\left\langle\left.\operatorname{exec}\right|_{S}, T_{0}\right\rangle \models \psi \sigma$. However, this result is wrong in general since the substitution $\sigma$ witnessing the fact that the attack formula $\phi$ is satisfiable can use some terms that only occur in events coming from sessions that are not in $S$. Thus, the first step of the proof consists in showing that we can consider a substitution $\sigma$ that only involves subterms that occur in $\mathrm{St}\left(\left.\mathrm{exec}\right|_{S}\right)$. For instance, consider the formula $\exists x$.learn $(x)$. Since, the variable $x$ does not occur in any status event, we cannot ensure that $x$ will be bound to a term coming from a session in $S$. However, intuitively, we can replace such a term $x \sigma$ by a nonce in $\mathcal{N}_{\epsilon}$ still preserving the satisfiability of the attack formula. Now, we can 
assume w.l.o.g. that that for all $j \in\{1, \ldots, n\}, \sigma\left(x_{j}\right) \in \operatorname{St}(\operatorname{exec}, S) \cup \mathcal{A} \cup \lg K e y s \cup \mathcal{N}_{\epsilon}$. Then, we proceed by induction on the length of the execution trace and the size of the formula, and we show that $\left\langle\left.\operatorname{exec}\right|_{S}, T_{0}\right\rangle \models \psi \sigma$. In other words, the attack formula is satisfiable and $\sigma$ is a witness of this fact.

Proposition 7.7. Let $\Pi$ be a $k$-party protocol and $T_{0}$ be a finite set of ground atoms such that lgKeys $(\Pi) \cap$ plaintext $(\Pi) \subseteq T_{0} \cup \mathcal{K}_{\epsilon}$. Let exec be a valid and well-formed execution of $\widetilde{\Pi}$ w.r.t. $T_{0}$, and $\phi=\exists x_{1} \ldots . \exists x_{n} . \psi$ be an attack formula such that $\left\langle\right.$ exec, $\left.T_{0}\right\rangle \models \psi \sigma$ for some ground substitution $\sigma$. We have that $\left\langle\right.$ exec $\left.\left.\right|_{S}, T_{0}\right\rangle \models \phi$ where $S=\{$ sid $\mid \exists$ sid $\in$ Ws $($ exec, $\psi \sigma)$ and sid $\in$ sameTagAs $\left(\right.$ exec, sid $\left.\left.{ }^{\prime}\right)\right\}$.

Proof. Let exec be a valid and well-formed execution of $\widetilde{\Pi}$ w.r.t. $T_{0}$ such that $\left\langle\right.$ exec, $\left.T_{0}\right\rangle \models \phi$. Claim: $T_{0} \cup \mathrm{K}($ exec $) \not k$ for any $k \in \lg$ Keys $\backslash\left(\mathcal{K}_{\epsilon} \cup T_{0}\right)$. Assume that there exists $k \in \lg$ Keys such that $T_{0} \cup \mathrm{K}($ exec $) \vdash k$. Using Lemma 2.4, we obtain that $k \in \operatorname{plaintext}($ exec $) \cup T_{0} \cup \mathcal{K}_{\epsilon}$, and relying on Lemma 3.10, we conclude that $k \in$ plaintext(tr) $\cup T_{0} \cup \mathcal{K}_{\epsilon}$ where $\operatorname{tr}$ is the symbolic trace underlying exec. Now, by construction of $\operatorname{tr}$, if $k \in$ plaintext(tr), then there exists $k^{\prime} \in$ plaintext $(\Pi)$ such that $k=k^{\prime} \sigma$ for some $\sigma: \mathcal{X} \rightarrow \mathcal{A}$. Hence, we have that $k^{\prime} \in \operatorname{lgKeys}(\Pi) \cup$ plaintext $(\Pi)$. Thanks to our hypothesis, we conclude that $k^{\prime} \in T_{0} \cup \mathcal{K}_{\epsilon}$, and thus $k^{\prime}=k \in T_{0} \cup \mathcal{K}_{\epsilon}$, which concludes the proof of the claim.

By hypothesis, we have that $\left\langle\right.$ exec, $\left.T_{0}\right\rangle \models \psi \sigma$ for some ground substitution $\sigma$. Moreover, by hypothesis, we have that:

(1) Ws $($ exec, $\psi \sigma) \subseteq S$, and

(2) $\forall$ sess $_{1}$, sess $_{2}$ with ExpectedTag $($ exec, sess 1$)=$ ExpectedTag $_{1}$ exec, sess 2$)$, we have that $\operatorname{sess}_{1} \in S$ if and only if $\operatorname{sess}_{2} \in S$.

Hence, we can apply Lemma 7.6 to conclude that $\left\langle\left.\operatorname{exec}\right|_{S}, T_{0}\right\rangle \models \phi$.

\section{MAin Results}

In this section, we put the pieces together and prove Theorem 5.6, the main result that was stated in Section 5.2, We also prove Corollary 1 which allows us to obtain slightly stronger results for particular security properties.

To prove our main result, we first need to bound the number of sessions that are needed to witness the satisfiability of the attack formula under study. This is the purpose of the following lemma that can be proved by induction on the structure of $\phi$.

Lemma 8.1. Let $\Pi$ be a protocol, $\phi$ a closed quantifier-free formula of $\mathcal{L}$, and $T_{0}$ be set of ground atoms. Let exec $=\left[\mathrm{e}_{1}^{s^{i d_{1}}} ; \ldots ; \mathrm{e}_{\ell}^{\text {sid }}\right]$ be a valid execution of $\Pi$ (w.r.t. $T_{0}$ ) satisfying $\phi$, i.e. $\left\langle\right.$ exec, $\left.T_{0}\right\rangle \models \phi$. We have that $|\mathrm{Ws}(\operatorname{exec}, \phi)| \leq\|\phi\|$. 


\subsection{Proof of Theorem 5.6. Now, we prove our main theorem.}

Theorem 5.6. Let $\Pi$ be a k-party protocol, $\widetilde{\Pi}$ be its corresponding transformed protocol and $T_{0}$ be a set of ground atoms such that lgKeys $(\Pi) \cap$ plaintext $(\Pi) \subseteq T_{0} \cup \mathcal{K}_{\epsilon}$. Let $\phi$ be an attack formula such that $\widetilde{\Pi}=\phi$ w.r.t. $T_{0}$. There exists a valid execution trace exec of $\widetilde{\Pi}$ such that:

$$
\left\langle\text { exec, } T_{0}\right\rangle \models \phi \text { and exec involves at most }\|\phi\| \text { sessions of each role. }
$$

Proof. Let exec be a valid execution of $\widetilde{\Pi}$ w.r.t. $T_{0}$ such that $\left\langle\right.$ exec, $\left.T_{0}\right\rangle \models \phi$. By Proposition 6.13 we have that $\overline{\text { exec }}$ is a valid well-formed execution of $\widetilde{\Pi}$ w.r.t. $T_{0}$, and according to Proposition 6.14, we have that $\left\langle\overline{\mathrm{exec}}, T_{0}\right\rangle \models \phi$. By definition on an attack formula, we have that $\phi=\exists x_{1} \ldots . \exists x_{n} . \psi$ and we deduce that there exists $\sigma$ such that $\left\langle\overline{\mathrm{exec}}, T_{0}\right\rangle \models \psi \sigma$.

Let $S=\left\{\right.$ sid $\mid \exists s i d^{\prime} \in \mathrm{Ws}(\overline{\mathrm{exec}}, \psi \sigma)$ and sid $\in \operatorname{sameTagAs}\left(\overline{\mathrm{exec}}\right.$, sid $\left.\left.^{\prime}\right)\right\}$. Now, by Proposition [7.4, we have that $\left.\overline{\mathrm{exec}}\right|_{S}$ is also a well-formed and valid execution of $\widetilde{\Pi}$ w.r.t. $T_{0}$; and according to Proposition 7.7, we know that $\left\langle\left.\overline{\mathrm{exec}}\right|_{S}, T_{0}\right\rangle \models \phi$. Finally, Lemma 8.1 tells us that $|\mathrm{Ws}(\overline{\mathrm{exec}}, \psi \sigma)| \leq\|\phi \sigma\|=\|\phi\|$. But because by construction of $\widetilde{\Pi}$ (and hence of all of its symbolic traces), in every execution of $\widetilde{\Pi}$ all sessions of the same role are tagged differently (each session introduces its own nonce making them different), $S$ must contain at most $\|\phi\|$ sessions of each role. This allows us to conclude that $\left.\overline{\operatorname{exec}}\right|_{S}$ is an attack that involves at most $\|\phi\|$ sessions of each role.

8.2. Secrecy, aliveness and weak agreement. For several classical security properties we are actually able to obtain a slightly stronger result and only consider one honest session of each role. As we will see below this is a direct corollary from the proof of the main theorem.

Corollary 1. Let $\Pi$ be a $k$-party protocol, $\Pi_{\mathrm{S}}$ (respectively, $\Pi_{\mathrm{A}}, \Pi_{\mathrm{WA}}$ ) be the annotated protocol for modeling secrecy (respectively aliveness and weak agreement) as defined in Section 4.2.1, and $\widetilde{\Pi}_{\mathrm{S}}$ (respectively, $\widetilde{\Pi}_{\mathrm{A}}, \widetilde{\Pi}_{\mathrm{WA}}$ ) the corresponding transformed protocol. Let $T_{0}$ be a set of ground atoms such that lgKeys $(\Pi) \cap$ plaintext $(\Pi) \subseteq T_{0} \cup \mathcal{K}_{\epsilon}$ and $\overline{\phi_{\mathrm{S}}}$ (respectively $\overline{\phi_{\mathrm{A}}}, \overline{\phi_{\mathrm{WA}}}$ ) an attack formula against secrecy (respectively aliveness and weak agreement) as defined in Section 4.2.1. For $\mathrm{X} \in\{\mathrm{S}, \mathrm{A}, \mathrm{WA}\}$ we have that if $\widetilde{\Pi}_{\mathrm{X}} \models \overline{\phi_{\mathrm{X}}}$ w.r.t. $T_{0}$ then there exists a valid execution trace exec of $\widetilde{\Pi}_{X}$ such that:

$$
\left\langle\text { exec, } T_{0}\right\rangle \models \overline{\phi_{\mathrm{X}}} \text { and exec involves at most one honest session of each role. }
$$

Proof. We only detail the proof in the case of secrecy. The case of aliveness and weak agreement are treated similarly. Let $\overline{\phi_{\mathrm{S}}}=\exists x_{1} \ldots . \exists x_{n} . \exists y \cdot \overline{\psi_{\mathrm{S}}}$. Following the proof of Theorem [5.6, we can show that $\left\langle\overline{\mathrm{exec}}, T_{0}\right\rangle \models \overline{\psi_{\mathrm{S}}} \sigma$ for some substitution $\sigma$.

Let $S=\left\{\right.$ sid $\mid \exists$ sid ${ }^{\prime} \in \mathrm{Ws}\left(\overline{\mathrm{exec}}, \overline{\psi_{\mathrm{S}}} \sigma\right)$ and sid $\left.\in \operatorname{sameTagAs}\left(\overline{\mathrm{exec}}, \operatorname{sid}^{\prime}\right)\right\}$. We have that $\mathrm{Ws}\left(\overline{\mathrm{exec}}, \overline{\psi_{\mathrm{S}}} \sigma\right)=1$, and thus the set $S$ contains at most one session of each role. To conclude, we have to show that $S$ only contains honest sessions. By definition of Ws, we know that $\mathrm{Ws}\left(\overline{\mathrm{exec}}, \overline{\psi_{\mathrm{S}}} \sigma\right)=\left\{\operatorname{sid}_{0}\right\}$ for some $\operatorname{sid}_{0}$ such that the status event $\operatorname{Secret}\left(x_{1} \sigma, \ldots, x_{k} \sigma, y \sigma\right)$ is issued from the session $\operatorname{sid}_{0}$ and we have that $\left\langle\overline{\mathrm{exec}}, T_{0}\right\rangle \models \mathrm{NC}\left(x_{1} \sigma\right) \wedge \ldots \wedge \mathrm{NC}\left(x_{k} \sigma\right)$. Hence, we have that $\operatorname{sid}_{0}$ is an honest session.

We have that $S=\left\{\right.$ sid $\mid \exists$ sid $\in \mathrm{Ws}\left(\overline{\mathrm{exec}}, \overline{\psi_{\mathrm{S}}} \sigma\right)$ and sid $\in$ sameTagAs $\left(\overline{\mathrm{exec}}\right.$, sid $\left.\left.^{\prime}\right)\right\}$ which means that $S=\left\{\right.$ sid $\mid$ sid $\in$ sameTagAs $\left(\overline{\mathrm{exec}}\right.$, sid $\left.\left._{0}\right)\right\}$. Since the names of the agents that are 
involved in a session occur in the tag, we know that all the sessions in $S$ are honest. This allows us to conclude.

\section{Conclusion}

In this paper we present a transformation which guarantees that attacks on transformed protocols only require a number of sessions which is a function of the security property under study. We prove this result for a class of security properties that includes secrecy and several flavors of authentication. Our logic for specifying security properties does not allow one to express injective authentication properties (e.g. injective agreement, matching conversations, etc.) but we believe that both the logic and our reduction result could be extended to this setting.

A challenging topic for future research is to obtain more fine-grained characterizations of decidable classes of protocols for an unbounded number of sessions. The new insights gained by our work seem to be a good starting point to extract the conditions needed to reduce the security for an unbounded number of sessions to a finite number of sessions.

\section{ACKNOWLEDGMENTS}

This work has been partially supported by the projects JCJC VIP ANR-11-JS02-006 and ERC grant agreement no 258865, project ProSecure.

\section{REFERENCES}

[1] M. Arapinis. Sécurité des protocoles cryptographiques : décidabilité et résultats de réduction. Thèse de doctorat, Université Paris 12, Créteil, France, Nov. 2008.

[2] M. Arapinis, S. Delaune, and S. Kremer. From one session to many: Dynamic tags for security protocols. In I. Cervesato, H. Veith, and A. Voronkov, editors, Proceedings of the 15th International Conference on Logic for Programming, Artificial Intelligence, and Reasoning (LPAR'08), volume 5330 of Lecture Notes in Artificial Intelligence, pages 128-142, Doha, Qatar, 2008. Springer.

[3] M. Arapinis and M. Duflot. Bounding messages for free in security protocols. In Proc. 27th Conference on Foundations of Software Technology and Theoretical Computer Science (FSTETCS'07), volume 4855 of $L N C S$, pages 376-387. Springer, 2007.

[4] A. Armando et al. The Avispa tool for the automated validation of internet security protocols and applications. In Proc. 17th International Conference on Computer Aided Verification (CAV'05), volume 3576 of LNCS, pages 281-285. Springer, 2005.

[5] B. Barak, Y. Lindell, and T. Rabin. Protocol initialization for the framework of universal composability. Cryptology ePrint Archive, Report 2004/006, 2004.

[6] D. Beauquier and F. Gauche. How to guarantee secrecy for cryptographic protocols. CoRR, abs/cs/0703140, 2007.

[7] M. Bellare, R. Canetti, and H. Krawczyk. A modular approach to the design and analysis of authentication and key exchange protocols (extended abstract). In Proc. 30th Annual ACM Symposium on the Theory of Computing (STOC'98), pages 419-428. ACM Press, 1998.

[8] B. Blanchet. An efficient cryptographic protocol verifier based on prolog rules. In Proc. 14th Computer Security Foundations Workshop (CSFW'01), pages 82-96, Cape Breton (Canada), 2001. IEEE Comp. Soc. Press.

[9] B. Blanchet and A. Podelski. Verification of cryptographic protocols: Tagging enforces termination. In Proc. Foundations of Software Science and Computation Structures (FoSSaCS'03), volume 2620 of LNCS, pages 136-152. Springer, 2003. 
[10] R. Canetti. Universally composable security: A new paradigm for cryptographic protocols. In Proc. 42nd Annual Symposium on Foundations of Computer Science (FOCS'01), pages 136-145, Las Vegas (Nevada, USA), 2001. IEEE Comp. Soc.

[11] C. Chevalier, S. Delaune, and S. Kremer. Transforming password protocols to compose. In S. Chakraborty and A. Kumar, editors, Proceedings of the 31st Conference on Foundations of Software Technology and Theoretical Computer Science (FSTTCS'11), Leibniz International Proceedings in Informatics, pages 204-216. Leibniz-Zentrum für Informatik, 2011.

[12] Ş. Ciobâcă and V. Cortier. Protocol composition for arbitrary primitives. In Proceedings of the 23rd IEEE Computer Security Foundations Symposium (CSF'10), pages 322-336. IEEE Computer Society Press, July 2010.

[13] J. Clark and J. Jacob. A survey of authentication protocol literature. http://www.cs.york.ac.uk/ jac/papers/drareviewps.ps, 1997.

[14] H. Comon-Lundh and V. Cortier. Security properties: two agents are sufficient. Science of Computer gramming, 50(1-3):51-71, March 2004.

[15] H. Comon-Lundh, V. Cortier, and E. Zălinescu. Deciding security properties for cryptographic protocols. application to key cycles. ACM Trans. Comput. Logic, 11(2):1-42, 2010.

[16] R. Corin. Analysis Models for Security Protocols. PhD thesis, University of Twente, 2006.

[17] V. Cortier and S. Delaune. Safely composing security protocols. Formal Methods in System Design, 34(1):1-36, feb 2009.

[18] V. Cortier, B. Warinschi, and E. Zălinescu. Synthesizing secure protocols. In Proc. 12th European Symposium On Research In Computer Security (ESORICS'07), volume 4734 of LNCS, pages 406-421. Springer, 2007.

[19] D. Dolev, S. Even, and R. M. Karp. On the security of ping-pong protocols. In Proc. Advances in Cryptology (CRYPTO'82), pages 177-186, 1982.

[20] D. Dolev and A. C. Yao. On the security of public key protocols. In Proc. of the 22nd Symposium on Foundations of Computer Science (FOCS'81), pages 350-357. IEEE Comp. Soc. Press, 1981.

[21] N. Durgin, P. Lincoln, J. Mitchell, and A. Scedrov. Undecidability of bounded security protocols. In Proc. Workshop on Formal Methods and Security Protocols, 1999.

[22] J. Heather, G. Lowe, and S. Schneider. How to prevent type flaw attacks on security protocols. In Proc. 13th Computer Security Foundations Workshop (CSFW'01), pages 255-268. IEEE Comp. Soc. Press, 2000 .

[23] J. Katz and M. Yung. Scalable protocols for authenticated group key exchange. In Proc. 23rd Annual International Cryptology Conference (CRYPTO'03), volume 2729 of LNCS, pages 110-125. Springer, 2003.

[24] G. Lowe. Breaking and fixing the Needham-Schroeder public-key protocol using FDR. In Proc. 2nd International Workshop on Tools and Algorithms for the Construction and Analysis of Systems (TACAS'96), volume 1055 of LNCS, pages 147-166, Berlin (Germany), 1996. Springer.

[25] G. Lowe. A hierarchy of authentication specifications. In CSFW'97: Proceedings of the 10th IEEE workshop on Computer Security Foundations, page 31, Washington, DC, USA, 1997. IEEE Computer Society.

[26] G. Lowe. Towards a completeness result for model checking of security protocols. Journal of Computer Security, 7(1), 1999.

[27] R. Needham and M. Schroeder. Using encryption for authentication in large networks of computers. Communication of the ACM, 21(12):993-999, 1978.

[28] R. Ramanujam and S. P. Suresh. Tagging makes secrecy decidable for unbounded nonces as well. In Proc. 23rd Conference on Foundations of Software Technology and Theoretical Computer Science (FSTETCS'03), volume 2914 of LNCS, pages 363-374. Springer, 2003.

[29] R. Ramanujam and S. P. Suresh. Decidability of context-explicit security protocols. Journal of Computer Security, 13(1):135-165, 2005.

[30] M. Rusinowitch and M. Turuani. Protocol insecurity with finite number of sessions and composed keys is NP-complete. Theoretical Computer Science, 299(1-3):451-475, 2003. 


\section{Appendix A. Proofs of Section 6.2}

In this section, we show that our transformation maps an execution trace exec to a wellformed execution trace $\overline{\text { exec. }}$. The resulting execution trace $\overline{\text { exec }}$ is still a trace associated to the protocol $\widetilde{\Pi}$ under study.

Lemma A.1. Let $\Pi$ be a $k$-party protocol, and exec be an execution trace associated to $\widetilde{\Pi}$ (not necessarily a valid one). We have that $\overline{\mathrm{exec}}$ is an execution trace (not necessarily a valid one) associated to the protocol $\widetilde{\Pi}$

Proof. (sketch) Let $\operatorname{tr}=\left[\mathrm{e}_{1}^{s i d_{1}} ; \ldots ; \mathrm{e}_{\ell}^{\text {sid }}\right]$ be the symbolic trace of $\widetilde{\Pi}$, and $\sigma$ be the ground substitution such that $\operatorname{dom}(\sigma)=\operatorname{vars}(\operatorname{tr})$ and exec $=\operatorname{tr} \sigma$. Let $\bar{\sigma}$ be such that:

- $\operatorname{dom}(\bar{\sigma})=\operatorname{dom}(\sigma)$, and

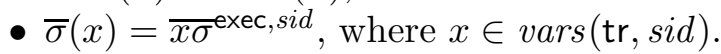

Clearly, we have that $\bar{\sigma}$ is a ground substitution. It remains to establish that $\overline{\mathrm{exec}}=\operatorname{tr} \bar{\sigma}$ so that the execution exec will rely on the same scenario than exec.

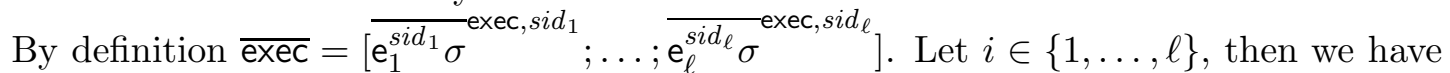
that $\mathrm{e}_{i}^{s i d_{i}}=\operatorname{rcv}(u)\left(\right.$ or $\mathrm{e}_{i}^{s i d_{i}}=\operatorname{snd}(u)$, or $\left.\mathrm{e}_{i}^{s i d_{i}}=\mathrm{Q}\left(u_{1}, \ldots, u_{n}\right)\right)$. Since the three cases can be handled in a similar way, we consider here the case where $e_{i}^{s i d_{i}}=\operatorname{rcv}(u)$. By definition, we have that ${\overline{\mathrm{e}_{i} \text { sid }_{i} \sigma}}^{\text {exec, sid }}{ }_{i}=\operatorname{rcv}\left(\overline{u \sigma}^{\text {exec, sid }}\right)$, and we prove by structural induction on $u^{\prime} \in \operatorname{St}(u)$ that ${\overline{u^{\prime} \sigma}}^{\text {exec,sidi }}=u^{\prime} \bar{\sigma}$. Finally, from this we conclude that $\overline{u \sigma}^{\mathrm{exec}, s i d_{i}}=u \bar{\sigma}$, and thus that ${\overline{\mathrm{e}_{i}^{s i d_{i}} \sigma}}^{\mathrm{exec}, s i d_{i}}=\operatorname{rcv}\left(\overline{u \sigma}^{\mathrm{exec}, s i d_{i}}\right)=\operatorname{rcv}(u \bar{\sigma})=\mathrm{e}_{i}^{s i d_{i}} \bar{\sigma}$. By definition, this brings us to $\overline{\mathrm{exec}}=\operatorname{tr} \bar{\sigma}$.

Lemma A.2. Let $\Pi$ be a k-party protocol, and exec be an execution trace associated to $\widetilde{\Pi}$ (not necessarily a valid one). We have that $\overline{\mathrm{exec}}$ is well-formed.

Proof. (sketch) Let exec $=\mathrm{e}_{1}^{\text {sid }}, \ldots, \mathrm{e}_{\ell}^{\text {sid }}$. Let $i \in\{1, \ldots, \ell\}$, we show that:

(1) ${\overline{\mathrm{e}_{i}}}^{\text {sid }_{i}}{ }^{\mathrm{exec}, s i d_{i}}$ is $k$-tagged;

(2) $\operatorname{Tags}\left({\overline{\mathrm{e}_{i}}}^{\text {id }_{i}}{ }^{\text {exec }, s_{i}}\right) \subseteq\left\{\operatorname{ExpectedTag}\left(\overline{\operatorname{exec}}, s_{i} d_{i}\right)\right\}$;

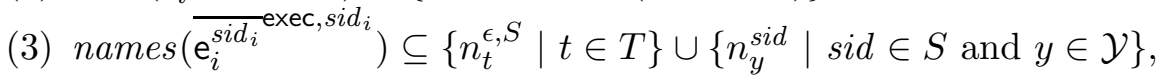
where $S=$ sameTagAs (exec, sid $\left._{i}\right)$.

Let $\mathrm{e}_{i}^{s i d_{i}}=\operatorname{rcv}(u)$ for some term $u$. The cases where $\mathrm{e}_{i}^{s i d_{i}}=\operatorname{snd}(u)$ or $\mathrm{e}_{i}^{s i d_{i}}=\mathrm{Q}\left(u_{1}, \ldots, u_{n}\right)$ can be done in a similar way. We have that ${\overline{\mathrm{e}_{i}^{\text {sid }}}}^{\text {exec, sid }}{ }^{\mathrm{erv}}=\operatorname{ru}\left(\bar{u}^{\mathrm{exec}, s i d_{i}}\right)$ and we prove by structural induction on $u^{\prime} \in \mathrm{St}(u)$ that:

(1) ${\overline{u^{\prime}}}^{\text {exec,sidi }}$ is $k$-tagged;

(2) $\operatorname{Tags}\left({\overline{u^{\prime}}}^{\text {exec, } \text { sid }_{i}}\right) \subseteq\left\{\right.$ ExpectedTag $\left.\left(\overline{\text { exec }}, \operatorname{sid}_{i}\right)\right\}$;

(3) names $\left({\overline{u^{\prime}}}^{\text {exec, sid }}\right) \subseteq\left\{n_{t}^{\epsilon, S} \mid t \in T\right\} \cup\left\{n_{y}^{\text {sid }} \mid\right.$ sid $\in S$ and $\left.y \in \mathcal{Y}\right\}$, where $S=$ sameTagAs(exec, sid $\left._{i}\right)$. 
And from this we derive that $\bar{u}^{\mathrm{exec}, s i d_{i}}$ satisfies the three conditions of well-formedness, and thus so is $\overline{\mathrm{e}}_{i}^{\text {sidi }_{i}}$ exec,sidi $_{\text {for }}$ for all $i \in\{1, \ldots, \ell\}$, which in turn implies by definition that $\overline{\text { exec }}$ satisfies the three conditions of well-formedness and is thus well-formed.

\section{Appendix B. Technical proofs about alien subterms}

We introduce the notion of alien subterms and we show that they satisfy some good properties. Later on, we will see that those alien subterms correspond to the subterms that are abstracted by our transformation - and we will use the properties established on them to prove the validity of the trace obtained after transformation.

Definition B.1 $\left(\mathrm{St}_{\mathrm{alien}}(\mathrm{exec}, \tau, t)\right)$. Let $\Pi$ be a $k$-party protocol and exec $=\left[\mathrm{e}_{1}^{s i d_{1}} ; \ldots ; \mathrm{e}_{\ell}^{\text {sid }_{\ell}}\right]$ be an execution trace (not necessarily valid) of $\widetilde{\Pi}$. We define the alien subterms of a term $t$ w.r.t. the execution exec and the active tag $\tau$, denoted $\mathrm{St}_{\text {alien }}(\mathrm{exec}, \tau, t)$, as follows:

- St $\mathrm{t}_{\text {alien }}(\mathrm{exec}, \tau, n)=\{n\}$ if $n \in \mathcal{N}_{\epsilon}$

- St $\mathrm{t}_{\text {alien }}\left(\right.$ exec, $\left.\tau, n_{y}^{\text {sid }}\right)= \begin{cases}\emptyset & \text { if ExpectedTag }(\text { exec, sid })=\tau \text { and } \tau \neq \perp \\ \left\{n_{y}^{\text {sid }}\right\} & \text { otherwise }\end{cases}$

- St $\mathrm{t}_{\mathrm{alien}}(\mathrm{exec}, \tau, a)=\emptyset$ if $a$ is an agent name

- St $t_{\text {alien }}\left(\right.$ exec, $\left.\tau, \mathrm{f}\left(a_{1}, \ldots, a_{n}\right)\right)=\emptyset$ if $\mathrm{f} \in\{$ shk, pub, priv $\}$

- St $\mathrm{t}_{\mathrm{alien}}(\operatorname{exec}, \tau,\langle u, v\rangle)=\mathrm{St}_{\mathrm{alien}}(\mathrm{exec}, \tau, u) \cup \mathrm{St}_{\mathrm{alien}}(\mathrm{exec}, \tau, v)$

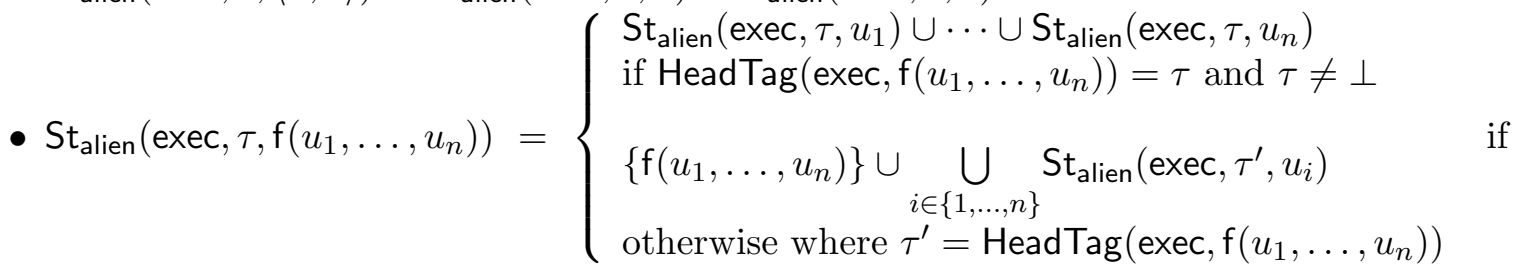

$\mathrm{f} \in\{$ encs, enca, sign, $\mathrm{h}\}$.

We define $\mathrm{St}_{\mathrm{alien}}(\mathrm{exec}, t)=\mathrm{St}_{\mathrm{alien}}(\mathrm{exec}, \perp, t)$, and extend this notion to sets of terms in the obvious way, i.e. $\mathrm{St}_{\text {alien }}(\mathrm{exec}, T)=\bigcup_{t \in T} \mathrm{St}_{\mathrm{alien}}(\mathrm{exec}, t)$.

Definition B.2 (vars (exec, sid), St(exec, sid), names(exec, sid)). Let $\Pi$ be a $k$-party protocol and exec be an execution trace of $\widetilde{\Pi}$. Let sid be a session identifier, and $\left[\mathrm{e}_{1}^{\text {sid }} ; \ldots ; \mathrm{e}_{h}^{\text {sid }}\right] \stackrel{\text { def }}{=}$ exec| $\left.\right|_{\{\text {sid }\}}$. We define the variables, subterms, and names in exec of a session sid as follows:

$$
\begin{aligned}
\operatorname{vars}(\mathrm{exec}, \text { sid }) & =\left\{x \mid x \in \operatorname{vars}\left(\mathrm{e}_{j}^{\text {sid }}\right) \text { for some } j \in\{1, \ldots, h\}\right\} \\
\mathrm{St}(\mathrm{exec}, \text { sid }) & =\left\{u \mid u \in \operatorname{St}\left(\mathrm{e}_{j}^{\text {sid }}\right) \text { for some } j \in\{1, \ldots, h\}\right\} \\
\text { names }(\mathrm{exec}, \text { sid }) & =\left\{u \mid u \in \operatorname{names}\left(\mathrm{e}_{j}^{\text {sid }}\right) \text { for some } j \in\{1, \ldots, h\}\right\} .
\end{aligned}
$$

Since we do not tag the pairing function symbol, this function symbol has a special status. We denote by $\operatorname{comp}(t)$ the components of a term $t$. This notion is formally defined as follows:

Definition B.3 $(\operatorname{comp}(t))$. Let $t$ be a term, the set of components of $t$ is:

$$
\operatorname{comp}(t)=\left\{\begin{array}{lr}
\operatorname{comp}(u) \cup \operatorname{comp}(v) & \text { if } t=\langle u, v\rangle \\
\{t\} & \text { otherwise. }
\end{array}\right.
$$


Lemma B.4. Let $\Pi$ be a $k$-party protocol, exec be an execution trace of $\widetilde{\Pi}$, and $t$ be a term. For all $k$-tags $\tau$, we have that:

(1) $\mathrm{St}_{\mathrm{alien}}(\mathrm{exec}, \tau, t)=\bigcup_{t^{\prime} \in \operatorname{comp}(t)} \mathrm{St}_{\mathrm{alien}}\left(\mathrm{exec}, \tau, t^{\prime}\right)$;

(2) $\mathrm{St}_{\mathrm{alien}}(\mathrm{exec}, \tau, t) \subseteq \mathrm{St}_{\mathrm{alien}}(\mathrm{exec}, t)$;

(3) $\mathrm{St}_{\text {alien }}(\mathrm{exec}, t) \subseteq \operatorname{comp}(t) \cup \mathrm{St}_{\text {alien }}(\mathrm{exec}, \tau, t)$.

Proof. We prove each statement separately by induction on the depth of $t$.

Lemma B.5. Let $\Pi$ be a $k$-party protocol, exec be an execution trace of $\widetilde{\Pi}$, and $u$ be a term. For any $v \in \mathrm{St}(u)$, we have that $\mathrm{St}_{\mathrm{alien}}(\mathrm{exec}, v) \subseteq \mathrm{St}_{\mathrm{alien}}(\mathrm{exec}, u) \cup \operatorname{comp}(v)$.

Proof. We first need to establish the following result:

$$
\forall \tau^{\prime} \exists \tau \mathrm{St}_{\text {alien }}(\mathrm{exec}, \tau, v) \subseteq \mathrm{St}_{\mathrm{alien}}\left(\mathrm{exec}, \tau^{\prime}, u\right) .
$$

If $v=u$ then we can choose $\tau=\tau^{\prime}$ to prove what we want. Otherwise, we have that $v \neq u$, and we prove the result by induction on the depth of $u$, and for this we distinguish three cases:

Case $u=\mathrm{f}\left(u_{1}, \ldots, u_{n}\right)$ for some $\mathrm{f} \in\{$ pub, priv, shk $\}$. Then we have that $\operatorname{St}_{\text {alien }}(v)=\emptyset$ for any $v \in \operatorname{St}(u)$. This allows us to easily conclude.

Case $u=\left\langle u_{1}, u_{2}\right\rangle$. In that case $v \in \operatorname{St}\left(u_{1}\right)$ or $v \in \operatorname{St}\left(u_{2}\right)$. Suppose $v \in \operatorname{St}\left(u_{1}\right)$ and let $\tau^{\prime}$ be a tag. By induction hypothesis, we have that there exists $\tau$ such that $\mathrm{St}_{\text {alien }}(\mathrm{exec}, \tau, v) \subseteq$ $\mathrm{St}_{\text {alien }}\left(\right.$ exec, $\left.\tau^{\prime}, u_{1}\right)$. By Definition B.1, we have that $\mathrm{St}_{\text {alien }}\left(\operatorname{exec}, \tau^{\prime}, u_{1}\right) \subseteq \mathrm{St}_{\text {alien }}\left(\mathrm{exec}, \tau^{\prime}, u\right)$. Hence, we easily conclude. The case where $v \in \operatorname{St}\left(u_{2}\right)$ can be handled in a similar way.

Case $u=\mathrm{f}\left(u_{1}, \ldots, u_{n}\right)$ for some $\mathrm{f} \in\{$ encs, enca, sign, $\mathrm{h}\}$. In that case, we have that $v \in \operatorname{St}\left(u_{i_{0}}\right)$ for some $i_{0} \in\{1, \ldots, n\}$. Let $\tau^{\prime}$ be a $k$-tag. According to Definition B.1, we have that $\bigcup_{i \in\{1, \ldots, n\}} \mathrm{St}_{\text {alien }}\left(\right.$ exec, $\left.\tau^{\prime \prime}, u_{i}\right) \subseteq \mathrm{St}_{\text {alien }}\left(\right.$ exec, $\left.\tau^{\prime}, u\right)$ where $\tau^{\prime \prime}=\operatorname{HeadTag}(\operatorname{exec}, u)$. Moreover, by induction hypothesis, we know that there exists $\tau$ such that $\operatorname{St}_{\text {alien }}(\operatorname{exec}, \tau, v) \subseteq$ $\mathrm{St}_{\text {alien }}\left(\mathrm{exec}, \tau^{\prime \prime}, u_{i_{0}}\right)$. Hence, we deduce that $\mathrm{St}_{\text {alien }}(\mathrm{exec}, \tau, v) \subseteq \mathrm{St}_{\mathrm{alien}}\left(\mathrm{exec}, \tau^{\prime}, u\right)$.

This allows us to conclude that $\forall \tau^{\prime} \exists \tau$, St $\mathrm{t}_{\text {alien }}$ (exec, $\left.\tau, v\right) \subseteq \mathrm{St}_{\mathrm{alien}}$ (exec, $\tau^{\prime}, u$ ). We have shown that $\forall \tau^{\prime} \mathrm{St}_{\text {alien }}$ (exec, $\left.\tau^{\prime}, u\right) \subseteq \mathrm{St}_{\text {alien }}($ exec, $u$ ) (see Lemma B.4- Item 2). Hence, we can infer that there exists $\tau$ such that $\mathrm{St}_{\text {alien }}(\operatorname{exec}, \tau, v) \subseteq \mathrm{St}_{\mathrm{alien}}(\mathrm{exec}, u)$. Hence, we have that:

$$
\begin{aligned}
\mathrm{St}_{\text {alien }}(\mathrm{exec}, v) & \subseteq \mathrm{St}_{\mathrm{alien}}(\mathrm{exec}, \tau, v) \cup \operatorname{comp}(v) \quad \text { (Lemma B.4- Item 3) } \\
& \subseteq \mathrm{St}_{\text {alien }}(\mathrm{exec}, u) \cup \operatorname{comp}(v)
\end{aligned}
$$

Lemma B.6. Let $\Pi$ be a $k$-party protocol and exec be an execution trace of $\widetilde{\Pi}$. Let $T$ be a set of terms such that $T \vdash v$ for any $v \in \mathrm{St}_{\text {alien }}(\mathrm{exec}, T)$, and $t$ be a term such that $T \vdash t$. We have $T \vdash u$ for any $u \in \mathrm{St}_{\text {alien }}(\mathrm{exec}, t)$.

Proof. Let $u \in \mathrm{St}_{\text {alien }}$ (exec, $t$ ). We prove that $T \vdash u$ by induction on $\pi$, a prooftree witnessing the fact that $T \vdash t$. If $\pi$ is reduced to a leaf then we have that $t \in T \cup \mathcal{A} \cup \mathcal{K}_{\epsilon} \cup \mathcal{N}_{\epsilon} \cup$ $\{\operatorname{pub}(a) \mid a \in \mathcal{A}\}$. Actually, if $t \in \mathcal{A} \cup \mathcal{K}_{\epsilon} \cup \mathcal{N}_{\epsilon} \cup\{\operatorname{pub}(a) \mid a \in \mathcal{A}\}$, then $\mathrm{St}_{\text {alien }}(\mathrm{exec}, t)=\emptyset$, leading to a contradiction. Hence, we have that $t \in T$, and thus $u \in \mathrm{St}_{\text {alien }}(\mathrm{exec}, T)$. We can thus conclude by hypothesis that $T \vdash u$. 
Otherwise, we proceed by case analysis on the last rule used in the proof $\pi$.

Case 1: the last rule is a composition rule. Then $t=\mathrm{f}\left(t_{1}, \ldots, t_{n}\right)$ for some terms $t_{1}, \ldots, t_{n}$ and some $\mathrm{f} \in\{\langle$,$\rangle , encs, enca, sign, \mathrm{h}\}$. Let $\pi_{1}, \ldots, \pi_{n}$ be the direct subproofs of $\pi$. We have that $\pi_{i}$ is a proof of $T \vdash t_{i}$ for $i \in\{1, \ldots, n\}$. According to Definition B.1 of alien subterms, $\mathrm{St}_{\text {alien }}(\mathrm{exec}, t) \subseteq\{t\} \cup \underset{i \in\{1, \ldots, n\}}{\bigcup} \mathrm{St}_{\text {alien }}\left(\mathrm{exec}, \tau, t_{i}\right)$ for some $\tau$, and by Lemma B.4 (Item 2) we can thus infer that

$$
\mathrm{St}_{\text {alien }}(\mathrm{exec}, t) \subseteq\{t\} \cup \underset{i \in\{1, \ldots, n\}}{\bigcup} \mathrm{St}_{\text {alien }}\left(\mathrm{exec}, t_{i}\right) .
$$

If $u=t$, then by hypothesis we know that $T \vdash u$. On the other hand, if $u \in \mathrm{St}_{\text {alien }}\left(\mathrm{exec}, t_{i}\right)$ for some $i \in\{1, \ldots, n\}$, then we conclude by applying our induction hypothesis on $\pi_{i}$. In both cases, we have that $T \vdash u$.

Case 2: the last rule is a projection rule. Then $t=t_{i_{0}}$ for some terms $t_{1}, t_{2}$, and some $i_{0} \in\{1,2\}$. Let $\pi^{\prime}$ be the direct subproof of $\pi$. We have that $\pi$ is a proof of $T \vdash\left\langle t_{1}, t_{2}\right\rangle$. According to Definition B.1] of alien subterms, $\mathrm{St}_{\text {alien }}(\mathrm{exec}, t) \subseteq \mathrm{St}_{\mathrm{alien}}\left(\mathrm{exec},\left\langle t_{1}, t_{2}\right\rangle\right)$, i.e. $u \in \mathrm{St}_{\text {alien }}\left(\mathrm{exec},\left\langle t_{1}, t_{2}\right\rangle\right)$. We can thus conclude by applying our induction hypothesis on $\pi^{\prime}$ that $T \vdash u$.

Case 3: the last rule is another decomposition rule. In such a case, there exists $t^{\prime}$ such that one of the direct subproofs of $\pi$ is labeled with $\mathrm{f}\left(t, t^{\prime}\right)$. Let $\pi^{\prime}$ be such a proof. Thanks to Lemma B.5 we know that either $u \in \mathrm{St}_{\text {alien }}\left(\mathrm{exec}, \mathrm{f}\left(t, t^{\prime}\right)\right)$ or $u \in \operatorname{comp}(t)$. In the first case, we can conclude by applying our induction hypothesis on $\pi^{\prime}$ that $T \vdash u$. In the second case, we know that by application of the projection rules one can derive $u$ from $t$, hence $T \vdash u$.

Lemma B.7. Let $\Pi$ be a $k$-party protocol and exec be an execution trace of $\widetilde{\Pi}$ associated to the symbolic trace $\operatorname{tr}=\left[\mathrm{e}_{1}^{\text {sid }}{ }_{1} ; \ldots ; \mathrm{e}_{\ell}^{\text {sid }}\right]$. Let $\sigma$ be the substitution such that $\operatorname{dom}(\sigma)=\operatorname{vars}(\operatorname{tr})$ and exec $=\operatorname{tr} \sigma$.

$$
\forall t \in \mathrm{St}(\mathrm{tr}, \text { sid }) \quad \mathrm{St}_{\mathrm{alien}}(\mathrm{exec}, t \sigma) \subseteq \operatorname{comp}(t \sigma) \cup \bigcup_{x \in \operatorname{vars}(t)} \mathrm{St}_{\mathrm{alien}}(\mathrm{exec}, \tau, x \sigma)
$$

where $\tau=$ ExpectedTag $($ exec, sid $)$.

Proof. Let $t \in \mathrm{St}(\mathrm{tr}$, sid $)$ and $\tau=\operatorname{ExpectedTag}(\mathrm{exec}$, sid $)$. We show by structural induction on $t$ that

$$
\mathrm{St}_{\text {alien }}(\mathrm{exec}, t \sigma) \subseteq \operatorname{comp}(t \sigma) \cup \bigcup_{x \in \operatorname{vars}(t)} \mathrm{St}_{\mathrm{alien}}(\mathrm{exec}, \tau, x \sigma)
$$

We distinguish several cases.

Case $t \in \mathcal{Y}$. In such a case, we can easily conclude thanks to Lemma B.4 (Item 3). Indeed, we have that:

$$
\mathrm{St}_{\text {alien }}(\mathrm{exec}, t \sigma) \subseteq \operatorname{comp}(t \sigma) \cup \mathrm{St}_{\text {alien }}(\mathrm{exec}, \tau, t \sigma)=\operatorname{comp}(t \sigma) \cup \bigcup_{x \in \operatorname{vars}(t)} \mathrm{St}_{\mathrm{alien}}(\mathrm{exec}, \tau, x \sigma)
$$

Case $t \in \mathcal{N}$. Then $t \sigma=t, \mathrm{St}_{\text {alien }}(\mathrm{exec}, t \sigma)=\{t \sigma\}$, and $\operatorname{comp}(t \sigma)=\{t \sigma\}$. Thus, we have that:

$$
\mathrm{St}_{\text {alien }}(\operatorname{exec}, t \sigma)=\{t \sigma\} \subseteq \operatorname{comp}(t \sigma) \cup \bigcup_{x \in \text { vars }(t)} \mathrm{St}_{\text {alien }}(\mathrm{exec}, \tau, x \sigma)
$$


Case $t \in \mathcal{A}$ or $t=\mathrm{f}\left(a_{1}, \ldots, a_{n}\right)$ for some $\mathrm{f} \in\{$ shk, pub, priv $\}$. In such a case, $\operatorname{vars}(t)=\emptyset$ and thus $\mathrm{St}_{\text {alien }}(\mathrm{exec}, t \sigma)=\emptyset$. This allows us to conclude.

Case $t=\left\langle t_{1}, t_{2}\right\rangle$. Then we have that Stalien $($ exec, $t \sigma)=\mathrm{St}_{\mathrm{alien}}\left(\mathrm{exec}, t_{1} \sigma\right) \cup \mathrm{St}_{\mathrm{alien}}\left(\mathrm{exec}, t_{2} \sigma\right)$. Applying our induction hypothesis, we deduce that

$$
\mathrm{St}_{\text {alien }}\left(\mathrm{exec}, t_{i} \sigma\right) \subseteq \operatorname{comp}\left(t_{i} \sigma\right) \cup \bigcup_{x \in \operatorname{vars}\left(t_{i}\right)} \mathrm{St}_{\text {alien }}(\mathrm{exec}, \tau, x \sigma) \text { for } i \in\{1,2\} \text {. }
$$

Hence, we conclude that $\mathrm{St}_{\text {alien }}(\mathrm{exec}, t \sigma) \subseteq \operatorname{comp}(t \sigma) \cup \underset{x \in \operatorname{vars}(t)}{\bigcup} \mathrm{St}_{\text {alien }}(\mathrm{exec}, \tau, x \sigma)$

Case $t=\mathrm{f}\left(t_{1}, \ldots, t_{n}\right)$ for some $\mathrm{f} \in\{$ encs, enca, sign, $\mathrm{h}\}$. Let $\tau^{\prime}=$ HeadTag(exec, $\left.t \sigma\right)$. We have that $\mathrm{St}_{\mathrm{alien}}(\mathrm{exec}, t \sigma)=\{t \sigma\} \cup \cup \mathrm{St}_{\text {alien }}\left(\mathrm{exec}, \tau^{\prime}, t_{i} \sigma\right)$. By construction of $\mathrm{tr}$, for all $i \in\{1, \ldots, n\}$

subterms $u \in \operatorname{CryptSt}(\operatorname{tr}$, sid $)$, HeadTag $($ exec, $u \sigma)=\tau$, thus $\tau^{\prime}=\tau$. Thanks to Lemma B.4 (Item 2), we have that $\mathrm{St}_{\text {alien }}(\mathrm{exec}, t \sigma) \subseteq\{t \sigma\} \cup \underset{i \in\{1, \ldots, n\}}{\cup} \mathrm{St}_{\mathrm{alien}}\left(\mathrm{exec}, t_{i} \sigma\right)$. We have that $\operatorname{comp}(t \sigma)=\{t \sigma\}$ and thanks to our induction hypothesis we have for each $i \in\{1, \ldots, n\}$ the following inclusion

$$
\mathrm{St}_{\text {alien }}\left(\mathrm{exec}, t_{i} \sigma\right) \subseteq \operatorname{comp}\left(t_{i} \sigma\right) \cup \bigcup_{x \in \operatorname{vars}\left(t_{i}\right)} \mathrm{St}_{\mathrm{alien}}(\mathrm{exec}, \tau, x \sigma)
$$

Thus, St $t_{\text {alien }}(\operatorname{exec}, t \sigma) \subseteq \operatorname{comp}(t \sigma) \cup \underset{i \in\{1, \ldots, n\}}{\bigcup} \operatorname{comp}\left(t_{i} \sigma\right) \cup \underset{x \in \operatorname{vars}(t)}{\bigcup} \mathrm{St}_{\text {alien }}(\mathrm{exec}, \tau, x \sigma)$.

Now, in order to conclude, it remains to show that for all $u \in \mathrm{St}_{\text {alien }}(\mathrm{exec}, t \sigma)$, if $u \in$ $\bigcup \operatorname{comp}\left(t_{i} \sigma\right)$ then there exists $x \in \operatorname{vars}(t)$ such that $u \in \mathrm{St}_{\text {alien }}(\mathrm{exec}, \tau, x \sigma)$. First, we $i \in\{1, \ldots, n\}$ notice the following:

$$
\begin{array}{rlrl}
\mathrm{St}_{\mathrm{alien}}(\mathrm{exec}, t \sigma) & =\{t \sigma\} \cup \bigcup_{i \in\{1, \ldots, n\}} \mathrm{St}_{\mathrm{alien}}\left(\mathrm{exec}, \tau^{\prime}, t_{i} \sigma\right) & & \text { (Definition B.1) } \\
& =\{t \sigma\} \cup \bigcup_{i \in\{1, \ldots, n\}} \bigcup_{w \in \operatorname{comp}\left(t_{i} \sigma\right)} \mathrm{St}_{\mathrm{alien}}\left(\mathrm{exec}, \tau^{\prime}, w\right) & & \text { (Lemma B.4) } \\
& =\{t \sigma\} \cup \bigcup_{i \in\{1, \ldots, n\}} \bigcup_{v \in \operatorname{comp}\left(t_{i}\right)} \bigcup_{w \in \operatorname{comp}(v \sigma)} \mathrm{St}_{\mathrm{alien}}\left(\mathrm{exec}, \tau^{\prime}, w\right) & \text { (Definition B.3) } \\
& =\{t \sigma\} \cup \bigcup_{i \in\{1, \ldots, n\}} \bigcup_{v \in \operatorname{comp}\left(t_{i}\right)} \mathrm{St}_{\mathrm{alien}}\left(\mathrm{exec}, \tau^{\prime}, v \sigma\right) & & \text { (Lemma B.4) }
\end{array}
$$

Let $i \in\{1, \ldots, n\}$ be such that $u \in \mathrm{St}_{\text {alien }}(\operatorname{exec}, t \sigma)$ and $u \in \operatorname{comp}\left(t_{i} \sigma\right)$. In that case, according to the equation stated above, there exists $j \in\{1, \ldots, n\}$ such that $v \in \operatorname{comp}\left(t_{j}\right)$ and $u \in \mathrm{St}_{\text {alien }}\left(\mathrm{exec}, \tau^{\prime}, v \sigma\right)$. We now proceed by case analysis on $v$ :

- Case $v \in \mathcal{A}$ or $v=\mathrm{f}\left(a_{1}, \ldots, a_{n}\right)$ for some $f \in\{$ pub, priv, shk $\}$. In such a case, we have that $\mathrm{St}_{\text {alien }}\left(\right.$ exec, $\left.\tau^{\prime}, v \sigma\right)=\emptyset$. Thus, this case in not possible.

- Case $v \in \mathcal{N}$. In such a case, we have that $u=v$ and by construction of $\operatorname{tr}$ we have that $v=n_{y}^{\text {sid }}$ for some variable $y$. Since, $\tau=\tau^{\prime}$, we have that $\mathrm{St}_{\mathrm{alien}}\left(\mathrm{exec}, \tau^{\prime}, v \sigma\right)=\emptyset$. Thus, this case is not possible.

- Case $v=\mathrm{g}\left(v_{1}, \ldots, v_{m}\right)$ for some $\mathrm{g} \in\{$ encs, enca, sign, $\mathrm{h}\}$. In such a case, we have that $u=v \sigma$ and by construction of $\operatorname{tr}$ we know that HeadTag $($ exec, $v \sigma)=\operatorname{HeadTag}($ exec, $t \sigma)=$ $\tau\left(=\tau^{\prime}\right)$. Hence, we deduce that $v \sigma \notin \mathrm{St}_{\text {alien }}\left(\mathrm{exec}, \tau^{\prime}, v \sigma\right)$, and thus $u \notin \mathrm{St}_{\text {alien }}\left(\mathrm{exec}, \tau^{\prime}, v \sigma\right)$ leading again to a contradiction.

- Case $v$ is a variable. In such a case, we have that $v \in \operatorname{vars}\left(t_{j}\right) \subseteq \operatorname{vars}(t)$. Hence, we have the expected conclusion.

Altogether, this allows us to conclude that 


$$
\mathrm{St}_{\mathrm{alien}}(\mathrm{exec}, t \sigma) \subseteq \operatorname{comp}(t \sigma) \cup \bigcup_{x \in \operatorname{vars}(t)} \mathrm{St}_{\mathrm{alien}}(\mathrm{exec}, \tau, x \sigma)
$$

Lemma B.8. Let $\Pi$ be a k-party protocol and exec $=\left[\mathrm{e}_{1}^{\text {sid } d_{1}} ; \ldots ; \mathrm{e}_{\ell}^{\text {sid }}{ }_{\ell}\right]$ be an execution trace of $\widetilde{\Pi}$, w.r.t. some set $T_{0}$ of ground atoms, associated to the symbolic trace $\mathrm{tr}=$ $\left[\mathrm{ee}_{1}^{s i d_{1}} ; \ldots ; \mathrm{ee}_{\ell}^{\text {sid }}{ }_{\ell}\right]$. Let $\sigma$ be the substitution such that $\operatorname{dom}(\sigma)=\operatorname{vars}(\operatorname{tr})$ and $\operatorname{exec}=\operatorname{tr} \sigma$. Let sid be a session identifier, $x$ be a variable in vars $(\mathrm{tr}$, sid $), \tau=$ ExpectedTag(exec, sid), and $u \in \mathrm{St}(\mathrm{tr})$ such that $x \in \operatorname{vars}(u)$. We have that $\mathrm{St}_{\text {alien }}(\mathrm{exec}, \tau, x \sigma) \subseteq \mathrm{St}_{\text {alien }}(\mathrm{exec}, \tau, u \sigma)$.

Proof. Let $u$ be a subterm of $\operatorname{tr}$ such that $x \in \operatorname{vars}(u)$. We prove the result by structural induction on $u$. First, note that by construction of tr, we have that vars $\left(\operatorname{tr}, \operatorname{sid}^{\prime}\right) \cap$ $\operatorname{vars}\left(\operatorname{tr}, \operatorname{sid}^{\prime \prime}\right)=\emptyset$ when $\operatorname{sid}^{\prime} \neq \operatorname{sid}^{\prime \prime}$. Hence, for all $i \in\{1, \ldots, \ell\}$ if $x \in \operatorname{vars}\left(\mathrm{ee}_{i}^{\text {sid }_{i}}\right)$, then $\operatorname{sid}_{i}=$ sid; and thus, for all $i \in\{1, \ldots, \ell\}$ such that $u \in \operatorname{St}\left(\mathrm{ee}_{i}^{\text {sid }_{i}}\right)$, we know that $\operatorname{sid}_{i}=$ sid. Now, since $x \in \operatorname{vars}(u)$, we know that $u$ is not ground, and we only need to consider the three following cases:

Case $u \in \mathcal{Y}$. In this case $u=x$, and the result trivially holds.

Case $u=\left\langle u_{1}, u_{2}\right\rangle$ for some terms $u_{1}$ and $u_{2}$. In that case, $x \in \operatorname{vars}\left(u_{1}\right)$ or $x \in \operatorname{vars}\left(u_{2}\right)$. Assume that $x \in \operatorname{vars}\left(u_{1}\right)$. The other case can be handled in a similar way. By induction hypothesis, we know that $\mathrm{St}_{\mathrm{alien}}(\mathrm{exec}, \tau, x \sigma) \subseteq \mathrm{St}_{\mathrm{alien}}\left(\mathrm{exec}, \tau, u_{1} \sigma\right)$ and we have that $\mathrm{St}_{\text {alien }}\left(\mathrm{exec}, \tau, u_{i} \sigma\right) \subseteq \mathrm{St}_{\text {alien }}(\mathrm{exec}, \tau, u \sigma)$. Combining these two we easily conclude.

Case $u=\mathrm{f}\left(u_{1}, \ldots, u_{n}\right)$ for some $\mathrm{f} \in\{$ encs, enca, sign, $\mathrm{h}\}$ and some terms $u_{1}, \ldots, u_{n}$. In that case $x \in \operatorname{vars}\left(u_{i}\right)$ for some $i \in\{1, \ldots, n\}$. Let $j \in\{1, \ldots, n\}$ such that $x \in \operatorname{vars}\left(u_{j}\right)$. Now, by construction of tr, we know that HeadTag (exec, $u \sigma)=$ ExpectedTag (exec, sid), hence we have that $\mathrm{St}_{\text {alien }}\left(\operatorname{exec}, \tau, u_{j} \sigma\right) \subseteq \mathrm{St}_{\text {alien }}(\mathrm{exec}, \tau, u \sigma)$. Applying our induction hypothesis on $u_{j}$, we deduce that $\mathrm{St}_{\mathrm{alien}}(\operatorname{exec}, \tau, x \sigma) \subseteq \mathrm{St}_{\mathrm{alien}}\left(\operatorname{exec}, \tau, u_{j} \sigma\right)$. This allows us to conclude.

Now, we can show that the alien subterms that occur in a valid trace are deducible.

Lemma B.9. Let $\Pi$ be a $k$-party protocol and exec $\left.=\left[\mathrm{e}_{1}^{\text {sid }}{ }_{1} ; \ldots ; \mathrm{e}_{\ell}^{\text {sid }}\right]_{\ell}\right]$ be an execution trace of $\widetilde{\Pi}$ that is valid w.r.t. some set $T_{0}$ of ground atoms. Let $i \in\{0, \ldots, \ell\}$. We have that $\mathrm{K}\left(\operatorname{exec}_{i}\right) \cup T_{0} \vdash u$ for any $u \in \mathrm{St}_{\text {alien }}\left(\operatorname{exec} \mathrm{K}\left(\operatorname{exec}_{i}\right) \cup T_{0}\right)$.

Proof. Let $\operatorname{tr}=\left[\mathrm{ee}_{1}^{s i d_{1}} ; \ldots ; \mathrm{ee}_{\ell}^{\text {sid }} d_{\ell}\right]$ be the symbolic trace associated to exec. Let $\sigma$ be the substitution such that $\operatorname{dom}(\sigma)=\operatorname{vars}(\operatorname{tr})$ and exec $=\operatorname{tr} \sigma$. We prove the result by induction on $i$. The base case, where $i=0$ is obvious since $\mathrm{K}\left(\operatorname{exec}_{i}\right)=\emptyset$ and $\mathrm{St}_{\text {alien }}\left(\operatorname{exec}, T_{0}\right) \subseteq T_{0}$. Now, to deal with the inductive case, we distinguish three cases depending on the nature of the last event in $\operatorname{exec}_{i}$.

Case $\mathrm{e}_{i}^{\text {sid }}=\mathrm{P}\left(t_{1}, \ldots, t_{n}\right)$. Then, we have that $\mathrm{K}\left(\operatorname{exec}_{i}\right)=\mathrm{K}\left(\operatorname{exec}_{i-1}\right)$ and thus that $\mathrm{St}_{\text {alien }}\left(\right.$ exec, $\left.\mathrm{K}\left(\operatorname{exec}_{i}\right) \cup T_{0}\right)=\mathrm{St}_{\text {alien }}\left(\mathrm{exec} \mathrm{K}\left(\operatorname{exec}_{i-1}\right) \cup T_{0}\right)$. Thanks to our induction hypothesis, we know that $\left.\mathrm{K}\left(\operatorname{exec}_{i-1}\right) \cup T_{0} \vdash \mathrm{St}_{\text {alien }}\left(\operatorname{exec}, \mathrm{K}\left(\operatorname{exec}_{i-1}\right) \cup T_{0}\right)\right)$, thus we easily conclude.

Case $\mathrm{e}_{i}^{s i d_{i}}=\operatorname{rcv}(t)$. This case is similar to the previous one.

Case $\mathrm{e}_{i}^{\text {sid }}{ }_{i}=\operatorname{snd}(t)$. In such a case, we have that $\mathrm{ee}_{i}^{s i d_{i}}=\operatorname{snd}\left(t^{\prime}\right)$ for some term $t^{\prime}$ such that $t=t^{\prime} \sigma$. Let $\left.u \in \mathrm{St}_{\mathrm{alien}}\left(\mathrm{exec}, \mathrm{K}\left(\operatorname{exec}_{i}\right) \cup T_{0}\right)\right)$. The only case for which we can not easily conclude by applying our induction hypothesis is when $u \in \mathrm{St}_{\mathrm{alien}}(\mathrm{exec}, t)$. So, assume that 
$u \in \mathrm{St}_{\mathrm{alien}}(\mathrm{exec}, t)$. According to Lemma B.7, $u \in \operatorname{comp}\left(t^{\prime} \sigma\right) \cup \bigcup_{x \in \operatorname{vars}\left(t^{\prime}\right)} \mathrm{St}_{\text {alien }}(\mathrm{exec}, \tau, x \sigma)$ where $\tau=$ ExpectedTag $\left(\right.$ exec, sid $\left._{i}\right)$. We distinguish two cases:

(1) $u \in \operatorname{comp}\left(t^{\prime} \sigma\right)$. We have that $t^{\prime} \sigma=t \in \mathrm{K}\left(\operatorname{exec}_{i}\right)$ and thus $\mathrm{K}\left(\operatorname{exec}_{i}\right) \cup T_{0} \vdash u$.

(2) $u \in \mathrm{St}_{\text {alien }}(\operatorname{exec}, \tau, x \sigma)$ for some $x \in \operatorname{vars}\left(t^{\prime}\right)$ and $u \notin \operatorname{comp}\left(t^{\prime} \sigma\right)$. By the origination property we know that there exists $j<i$ such that $\operatorname{sid}_{j}=\operatorname{sid}_{i}, \mathrm{ee}_{j} \operatorname{sid}_{j}=\operatorname{rcv}\left(v^{\prime}\right)$ with $x \in \operatorname{vars}\left(v^{\prime}\right)$, and thus that $x \sigma \in \operatorname{St}\left(v^{\prime} \sigma\right)$. By Lemma B.8, we deduce that $u \in$ $\mathrm{St}_{\text {alien }}\left(\mathrm{exec}, \tau, v^{\prime} \sigma\right)$, and thanks to LemmaB.4 (Item 2), we have that $u \in \mathrm{St}_{\text {alien }}\left(\mathrm{exec}, v^{\prime} \sigma\right)$. We can then apply our induction hypothesis in order to deduce that $\mathrm{K}\left(\operatorname{exec}_{j-1}\right) \cup T_{0} \vdash$ $w$ for any $w \in \mathrm{St}_{\text {alien }}\left(\mathrm{exec}, \mathrm{K}\left(\operatorname{exec}_{j-1}\right) \cup T_{0}\right)$, and because exec is a valid trace, we have also that $\mathrm{K}\left(\operatorname{exec}_{j-1}\right) \cup T_{0} \vdash v^{\prime} \sigma$. Thus, according Lemma B.6, we deduce that $\mathrm{K}\left(\operatorname{exec}_{j-1}\right) \cup T_{0} \vdash w$ for any $w \in \mathrm{St}_{\text {alien }}\left(\operatorname{exec}, v^{\prime} \sigma\right)$. In particular, we conclude that $\mathrm{K}\left(\operatorname{exec}_{j-1}\right) \cup T_{0} \vdash u$.

\section{Appendix C. Proofs of section 6.3}

In order to show the validity of the resulting trace, we first characterize the subterms that are abstracted by our transformation. Actually, we can show that those subterms are alien subterms, and thus they enjoy the properties established in Appendix B.

Lemma C.1. Let $\Pi$ be a k-party protocol, exec be an execution trace of $\widetilde{\Pi}, t$ be a term and $p$ be a position. If there exists sid such that $\left.\left(\bar{t}^{\mathrm{exec}, s i d}\right)\right|_{p} \in \mathcal{N}_{\epsilon}$, then we have that $\left.t\right|_{p} \in \mathrm{St}_{\text {alien }}(\mathrm{exec}, t)$.

Proof. We will prove by induction on $p$ that $\left.t\right|_{p} \in \mathrm{St}_{\mathrm{alien}}(\mathrm{exec}, t)$.

Base case $p=\epsilon$. In that case, according to Definition 6.6, either $t \in \mathcal{N}$, or $t=\mathrm{f}\left(t_{1}, \ldots, t_{n}\right)$ for some $\mathrm{f} \in\{$ encs, enca, sign, $\mathrm{h}\}$. If $t \in \mathcal{N}$, we have that $\mathrm{St}_{\text {alien }}(\mathrm{exec}, t)=\{t\}$, and thus $\left.t\right|_{p}=\left.t\right|_{\epsilon} \in \mathrm{St} t_{\text {alien }}($ exec, $t)$. Otherwise, i.e. $t=\mathrm{f}\left(t_{1}, \ldots, t_{n}\right)$ for some $\mathrm{f} \in\{$ encs, enca, sign, $\mathrm{h}\}$, then we have that

$$
\mathrm{St}_{\mathrm{alien}}(\mathrm{exec}, t)=\{t\} \cup \underset{i \in\{1, \ldots, n\}}{\bigcup} \mathrm{St}_{\mathrm{alien}}\left(\mathrm{exec}, \tau, t_{i}\right)
$$

where $\tau=\operatorname{HeadTag}($ exec, $t)$. Hence, we have that $\left.t\right|_{p}=\left.t\right|_{\epsilon} \in \mathrm{St}_{\text {alien }}(\mathrm{exec}, t)$.

Inductive case $p=i_{0} \cdot q$. First, note that $t$ cannot be a long-term key, i.e. $t$ is not a term of the form $\operatorname{pub}\left(t^{\prime}\right), \operatorname{priv}\left(t^{\prime}\right)$ or shk $\left(t_{1}, t_{2}\right)$. Indeed, in such a case, $\left.\left(\bar{t}^{\text {exec,sid }}\right)\right|_{p} \notin \mathcal{N}_{\epsilon}$ for any sid. This would contradict one of our hypothesis. Thus, two cases remain:

Case $t=\left\langle t_{1}, t_{2}\right\rangle$. By Definition [6.6, $\bar{t}^{\text {exec }, \text { sid }}=\left\langle{\overline{t_{1}}}^{\text {exec,sid }},{\overline{t_{2}}}^{\text {exec }, \text { sid }}\right\rangle$. Suppose $i_{0}=1$. Then $\left.\left(\overline{t_{1}}{ }^{\text {exec,sid }}\right)\right|_{q} \in \mathcal{N}_{\epsilon}$, and thanks to our induction hypothesis we can derive that $\left.t_{1}\right|_{q} \in$ $\mathrm{St}_{\text {alien }}\left(\mathrm{exec}, t_{1}\right)$. We have that $\mathrm{St}_{\text {alien }}(\mathrm{exec}, t)=\mathrm{St}_{\mathrm{alien}}\left(\mathrm{exec}, t_{1}\right) \cup \mathrm{St}_{\mathrm{alien}}\left(\mathrm{exec}, t_{2}\right)$, thus $\left.t_{1}\right|_{q} \in$ $\mathrm{St}_{\text {alien }}(\mathrm{exec}, t)$. Finally, since $\left.t_{1}\right|_{q}=\left.t\right|_{1 \cdot q}=\left.t\right|_{p}$ we can conclude that $\left.t\right|_{p} \in \mathrm{St}_{\text {alien }}(\mathrm{exec}, t)$. The case where $i_{0}=2$ can be done in a similar way.

Case $t=\mathrm{f}\left(t_{1}, \ldots, t_{n}\right)$ for some $\mathrm{f} \in\{$ encs, enca, sign, $\mathrm{h}\}$. Since $\left.\left(\bar{t}^{\mathrm{exec}, s i d}\right)\right|_{i_{0} . q} \in \mathcal{N}_{\epsilon}$, we know that $\left.\left(\bar{t}^{\text {exec,sid }}\right)\right|_{\epsilon} \notin \mathcal{N}_{\epsilon}$. Hence, by Definition 6.6, we have that

- $\bar{t}^{\text {exec }, \text { sid }}=f\left({\overline{t_{1}}}^{\text {exec,sid }}, \ldots,{\overline{t_{n}}}^{\text {exec }, s i d}\right)$;

- HeadTag $($ exec, $t)=$ ExpectedTag $($ exec, sid $) \neq \perp$; and 
- $\left.\left(\bar{t}^{\mathrm{exec}, s i d}\right)\right|_{i_{0} . q}=\left.\left({\overline{t_{i_{0}}}}^{\mathrm{exec}, \text { sid }}\right)\right|_{q}$.

Hence, we have that $\left.\left(\overline{{t_{0}}^{\text {exec, sid }}}\right)\right|_{q} \in \mathcal{N}_{\epsilon}$. Thanks to our induction hypothesis, we deduce that $\left.t_{i_{0}}\right|_{q} \in \mathrm{St} t_{\text {alien }}\left(\mathrm{exec}, t_{i_{0}}\right)$. Applying Lemma B.5, we conclude that $\left.t_{i_{0}}\right|_{q} \in \operatorname{comp}\left(t_{i_{0}}\right) \cup$ St $\mathrm{t}_{\text {alien }}(\mathrm{exec}, t)$.

In order to conclude, it is sufficient to show that if $\left.t_{i_{0}}\right|_{q} \in \operatorname{comp}\left(t_{i_{0}}\right)$, then we also have that $\left.t_{i_{0}}\right|_{q} \in \mathrm{St}_{\text {alien }}(\mathrm{exec}, t)$. Assume that $\left.t_{i_{0}}\right|_{q} \in \operatorname{comp}\left(t_{i_{0}}\right)$. First, let $\tau=\operatorname{HeadTag}(\mathrm{exec}, t)$, thanks to Lemma B.4 (item 11), we have that:

$$
\mathrm{St}_{\text {alien }}(\text { exec, } t)=\{t\} \cup \bigcup_{j \in\{1, \ldots, n\}} \mathrm{St}_{\text {alien }}\left(\text { exec, } \tau, t_{j}\right)=\{t\} \cup \bigcup_{i=1}^{n} \bigcup_{t_{j}^{\prime} \in \operatorname{comp}\left(t_{j}\right)} \mathrm{St}_{\text {alien }}\left(\text { exec, } \tau, t_{j}^{\prime}\right)
$$

Hence, we have that $\mathrm{St}_{\text {alien }}\left(\mathrm{exec}, \tau,\left.\left(t_{i_{0}}\right)\right|_{q}\right) \subseteq \mathrm{St}_{\text {alien }}(\mathrm{exec}, t)$. To conclude, it is hence enough to show that $\left.t_{i_{0}}\right|_{q} \in \mathrm{St}$ alien $\left(\mathrm{exec}, \tau,\left.t_{i_{0}}\right|_{q}\right)$. Since $\left.\left(\bar{t}^{\mathrm{exec}, s i d}\right)\right|_{p}=\left.\left(\bar{t}^{\mathrm{exec}, s i d}\right)\right|_{i_{0} . q}=\left.\left({\overline{t_{i_{0}}}}^{\mathrm{exec}, s i d}\right)\right|_{q} \in$ $\mathcal{N}_{\epsilon}$, we need to distinguish three cases:

- Case $\left.\left(t_{i_{0}}\right)\right|_{q} \in \mathcal{N}_{\epsilon}$. In such a case, we have that $\mathrm{St}_{\text {alien }}\left(\right.$ exec, $\left.\left.\tau,\left.\left(t_{i_{0}}\right)\right|_{q}\right)=\left\{\left.\left(t_{i_{0}}\right)\right|_{q}\right)\right\}$.

- Case $\left.\left(t_{i_{0}}\right)\right|_{q} \in \mathcal{N} \backslash \mathcal{N}_{\epsilon}$. In such a case, we have that $\left.\left(t_{i_{0}}\right)\right|_{q}=n_{y}^{\text {sid }}$ for some sid $^{\prime} \notin$ sameTagAs(exec, sid), thus Stalien $\left(\right.$ exec, $\left.\left.\tau,\left.\left(t_{i_{0}}\right)\right|_{q}\right)=\left\{\left.\left(t_{i_{0}}\right)\right|_{q}\right)\right\}$.

- Case $\left.\left(t_{i_{0}}\right)\right|_{q}=\mathrm{g}\left(u_{1}, \ldots, u_{m}\right)$ for some $\mathrm{g} \in\{$ encs, enca, sign, $\mathrm{h}\}$. In such a case, we have that HeadTag $\left(\left.t_{i_{0}}\right|_{q}\right) \neq \tau$, and thus

$$
\left.\mathrm{St}_{\mathrm{alien}}\left(\operatorname{exec}, \tau,\left.\left(t_{i_{0}}\right)\right|_{q}\right)=\left\{\left.\left(t_{i_{0}}\right)\right|_{q}\right)\right\} \cup \underset{j \in\{1, \ldots, m\}}{\bigcup} \mathrm{St}_{\mathrm{alien}}\left(\operatorname{exec}, \operatorname{HeadTag}\left(\left.t_{i_{0}}\right|_{q}\right), u_{j}\right) \text {. }
$$

We show that our transformation preserves disequalities even if terms are not abstracted using the same session identifier. This result can be proved by structural induction on $\bar{m}^{\text {exec, sid }}$.

Lemma C.2. Let $\Pi$ be a k-party protocol and $\mathrm{exec}=\left[\mathrm{e}_{1}^{\text {sid } d_{1}} ; \ldots ; \mathrm{e}_{\ell}^{\text {sid } d_{\ell}}\right]$ be a valid execution trace of $\widetilde{\Pi}$, w.r.t. some initial intruder knowledge $T_{0}$. Let $m$ and $m^{\prime}$ be two terms such that $m \neq m^{\prime}$, and sid, sid' be two session identifiers. We have that $\bar{m}^{\mathrm{exec}, \text { sid }} \neq{\overline{m^{\prime}}}^{\mathrm{exec}, \text { sid }^{\prime}}$.

Lemma 6.12. Let $\Pi$ be a k-party protocol and $\mathrm{exec}=\left[\mathrm{e}_{1}^{\text {sid }}{ }_{1} ; \ldots ; \mathrm{e}_{\ell}^{\text {sid }}\right]_{\ell}$ be a valid execution trace of $\widetilde{\Pi}, w . r . t$. some set $T_{0}$ of ground atoms. Let $i \in\{0, \ldots, \ell\}$ and $t$ be a term such that $\mathrm{K}\left(\operatorname{exec}_{i}\right) \cup T_{0} \vdash t$. We have that $\mathrm{K}\left(\overline{\operatorname{exec}}_{i}\right) \cup T_{0} \vdash \bar{t}^{\mathrm{exec}, \text { sid }}$ for any sid.

Proof. Let $\operatorname{tr}=\left[\mathrm{ee}_{1}^{s i d_{1}} ; \ldots ; \mathrm{ee}_{\ell}^{s i d_{\ell}}\right]$ be the symbolic trace associated to exec and $\sigma$ be the substitution such that $\operatorname{dom}(\sigma)=\operatorname{vars}(\operatorname{tr})$ and $\operatorname{exec}=\operatorname{tr} \sigma$. Let $i \in\{0, \ldots, \ell\}$. Let $\pi$ be a simple proof of $\mathrm{K}\left(\operatorname{exec}_{i}\right) \cup T_{0} \vdash t$. We prove that $\mathrm{K}\left(\overline{\operatorname{exec}}_{i}\right) \cup T_{0} \vdash \bar{t}^{\text {exec, sid }}$ by induction on $(i, \pi)$. If $i=0$ and $\pi$ is a simple proof reduced to a leaf (possibly followed by some projection rules), then we have that $T_{0} \vdash t$, and $\pi$ is necessarily reduced to a leaf since $T_{0}$ only contains atomic terms. Let sid be a session identifier, we have that $\bar{t}^{\text {exec,sid }} \in\{t\} \cup \mathcal{N}_{\epsilon}$. This allows us to conclude that $T_{0} \vdash \bar{t}^{\text {exec,sid }}$ Now, we distinguish several cases depending on the last rule of $\pi$.

The proof $\pi$ ends with an instance of a composition rule, i.e. $t=\mathrm{f}\left(t_{1}, \ldots, t_{n}\right)$ for some $\mathrm{f} \in\{\langle$,$\rangle , encs, enca, sign, \mathrm{h}\}$ and some terms $t_{1}, \ldots, t_{n}$.

According to Definition [6.6. we have that $\bar{t}^{\text {exec, sid }} \in \mathcal{N}_{\epsilon} \cup\left\{\mathrm{f}\left({\overline{t_{1}}}^{\text {exec, sid }}, \ldots,{\overline{t_{n}}}^{\text {exec }, \text { sid }}\right)\right\}$. If $\bar{t}^{\text {exec,sid }} \in \mathcal{N}_{\epsilon}$, we easily conclude that $\mathrm{K}\left(\overline{\operatorname{exec}}_{i}\right) \cup T_{0} \vdash \bar{t}^{\text {exec,sid }}$. Otherwise, since $\pi$ 
ends with a composition rule, we have that $\mathrm{K}\left(\operatorname{exec}_{i}\right) \cup T_{0} \vdash t_{1}, \ldots, \mathrm{K}\left(\operatorname{exec}_{i}\right) \cup T_{0} \vdash$ $t_{n}$. Moreover, the simple proofs witnessing these facts are strict subproofs of $\pi$ that are also simple. Hence, we can apply our induction hypothesis in order to conclude that $\mathrm{K}\left(\overline{\operatorname{exec}}_{i}\right) \cup T_{0} \vdash{\overline{t_{1}}}^{\text {exec, sid }}, \ldots, \mathrm{K}\left(\overline{\operatorname{exec}}_{i}\right) \cup T_{0} \vdash{\overline{t_{n}}}^{\text {exec,sid }}$. This allows us to conclude that $\mathrm{K}\left(\overline{\operatorname{exec}}_{i}\right) \cup T_{0} \vdash \mathrm{f}\left({\overline{t_{1}}}^{\mathrm{exec}, \text { sid }}, \ldots,{\overline{t_{n}}}^{\mathrm{exec}, \text { sid }}\right)$.

The proof ends with the application of a decomposition rule (but not a projection) possibly followed by several applications of the projection rules until the resulting term is not a pair. We will here present the case of the symmetric decryption rule, but all the other decomposition rules (including the case where the proof is reduced to a leaf) can be handled in a similar way. For some terms $t_{1}$ and $t_{2}$, the proof $\pi$ is of the form

$\frac{\frac{\vdots}{\mathrm{K}\left(\operatorname{exec}_{i}\right) \cup T_{0} \vdash \operatorname{encs}\left(t_{1}, t_{2}\right)} \quad \frac{\vdots}{\frac{\mathrm{K}\left(\operatorname{exec}_{i}\right) \cup T_{0} \vdash t_{1}}{\left.\operatorname{exec}_{i}\right) \cup T_{0} \vdash t_{2}}}}{\frac{\vdots}{\mathrm{K}\left(\operatorname{exec}_{i}\right) \cup T_{0} \vdash t}}$

Let us first note that, by locality (Lemma 6.11) and by simplicity of $\pi$ we know that encs $\left(t_{1}, t_{2}\right) \in \operatorname{St}\left(\mathrm{K}\left(\operatorname{exec}_{i}\right)\right) \cup T_{0} \cup \mathcal{K}_{\epsilon} \cup \mathcal{N}_{\epsilon} \cup\{\operatorname{pub}(a) \mid a \in \mathcal{A}\}$, and by atomicity of $T_{0}, \mathcal{N}_{\epsilon}, \mathcal{K}_{\epsilon}$ and $\{\operatorname{pub}(a) \mid a \in \mathcal{A}\}$, we know that encs $\left(t_{1}, t_{2}\right) \in \mathrm{St}(\mathrm{K}(\mathrm{exec}))$. (In case of a proof reduced to a leaf, and if there is no projection rule, we may have that $t \in T_{0}$. In such a case, as in the base case, we have that $T_{0} \vdash \bar{t}^{\text {exec,sid }}$ and we easily conclude.) Hence, there exists $k \leq i$ such that $\mathrm{e}_{k}^{s i d_{k}}=\operatorname{snd}(u)$ and encs $\left(t_{1}, t_{2}\right) \in \operatorname{St}(u)$. Let $k_{0}$ be the smallest such $k$ and $u_{0}, u_{0}^{\prime}$ be such that $\mathrm{e}_{k_{0}}^{s i d_{k_{0}}}=\operatorname{snd}\left(u_{0}\right)$ and $\mathrm{ee}_{k_{0}} \operatorname{sid}_{k_{0}}=\operatorname{snd}\left(u_{0}^{\prime}\right)$. Hence, we have that $u_{0}=u_{0}^{\prime} \sigma$. In order to prove the result, we first establish the following claim.

Claim: We have that $\overline{\operatorname{encs}\left(t_{1}, t_{2}\right)}{ }^{\text {exec, sid } k_{0}}=\operatorname{encs}\left({\overline{t_{1}}}^{\text {exec, } s i d_{k_{0}}},{\overline{t_{2}}}^{\text {exec, } s i d_{k_{0}}}\right)$.

Assume by contradiction, that this equality does not hold.

First, we have that encs $\left({\overline{t_{1}}}^{\text {exec,sid } k_{0}},{\overline{t_{2}}}^{\text {exec, sid }} k_{0}\right) \notin \mathrm{St}\left({\overline{u_{0}}}^{\text {exec, } s i d_{k_{0}}}\right)$. Indeed, for hav-

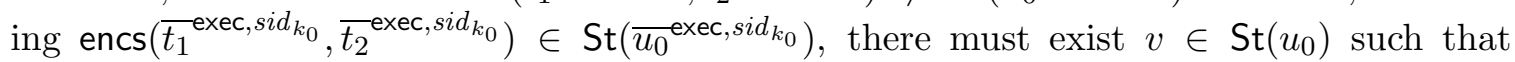
$\bar{v}^{\text {exec, } s i d_{k_{0}}}=\operatorname{encs}\left(\overline{t_{1}}{ }^{\text {exec,sid }} k_{0},{\overline{t_{2}}}^{\text {exec, } s i d_{k_{0}}}\right)$. But this would imply that $v=\operatorname{encs}\left(t_{1}^{\prime}, t_{2}^{\prime}\right)$ for some terms $t_{1}^{\prime}, t_{2}^{\prime}$ such that ${\overline{t_{1}^{\prime}}}^{\text {exec, sid } k_{0}}={\overline{t_{1}}}^{\text {exec,sid } k_{0}}$ and ${\overline{t_{2}^{\prime}}}^{\text {exec,sid } k_{0}}={\overline{t_{2}}}^{\text {exec,sid }}{ }_{k_{0}}$. However this would in turn imply according to Lemma C.2 that $t_{1}^{\prime}=t_{1}$ and $t_{2}^{\prime}=t_{2}$. In other words we would have $v=\operatorname{encs}\left(t_{1}, t_{2}\right) \in \operatorname{St}\left(u_{0}\right)$ but with $\bar{v}^{\text {exec, sid }} k_{k_{0}}=\operatorname{encs}\left(\overline{t_{1}}{ }^{\text {exec, sid }} k_{0},{\overline{t_{2}}}^{\text {exec, sid } k_{k_{0}}}\right)$

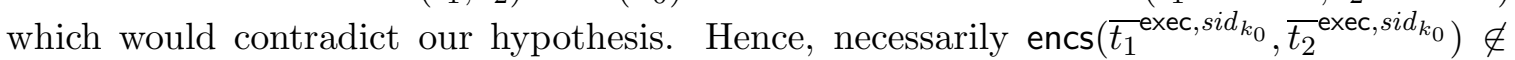
$\operatorname{St}\left({\overline{u_{0}}}^{\operatorname{exec}, s i d_{k_{0}}}\right)$.

Now since encs $\left({\overline{t_{1}}}^{\mathrm{exec}, s i d_{k_{0}}},{\overline{t_{2}}}^{\mathrm{exec}, \operatorname{sid}_{k_{0}}}\right) \notin \mathrm{St}\left({\overline{u_{0}}}^{\mathrm{exec}, s i d_{k_{0}}}\right)$, while encs $\left(t_{1}, t_{2}\right) \in \mathrm{St}\left(u_{0}\right)$, there must exist a position $p$ (smaller or equal to the position where encs $\left(t_{1}, t_{2}\right)$ occurs in $u_{0}$ ) such that $\left.\left(\overline{u_{0}}\right.$ exec,sid $\left.k_{0}\right)\right|_{p} \in \mathcal{N}_{\epsilon}$ and encs $\left(t_{1}, t_{2}\right)=\left.u_{0}\right|_{p}$. Hence, Lemma C.1 tells us that $\left.u_{0}\right|_{p} \in \mathrm{St}$ alien $\left(\right.$ exec, $u_{0}$ ). Thanks to Lemma B.7 and Lemma B.4 (Item 2), we conclude that:

$$
\left.u_{0}\right|_{p} \in \operatorname{comp}\left(u_{0}\right) \cup \bigcup_{x \in \operatorname{vars}\left(u_{0}^{\prime}\right)} \mathrm{St}_{\text {alien }}(\mathrm{exec}, x \sigma)
$$


We now distinguish two cases and show that each case leads us to a contradiction.

Case 1: $\left.u_{0}\right|_{p} \in \operatorname{comp}\left(u_{0}\right) \backslash \bigcup_{x \in \operatorname{vars}\left(u_{0}^{\prime}\right)} \mathrm{St}_{\mathrm{alien}}(\mathrm{exec}, x \sigma)$. In such a case, there exists $u_{0}^{\prime \prime} \in$ $\operatorname{comp}\left(u_{0}^{\prime}\right)$ such that $\left.u_{0}\right|_{p} \in \operatorname{comp}\left(u_{0}^{\prime \prime} \sigma\right)$. But because we are considering the case where $\left.u_{0}\right|_{p} \notin \bigcup_{x \in \operatorname{vars}\left(u_{0}^{\prime}\right)} \mathrm{St}_{\text {alien }}(\mathrm{exec}, x \sigma)$, it must be that $\left.u_{0}\right|_{p}=u_{0}^{\prime \prime} \sigma$. Now, by construction of tr, it must be that ExpectedTag (exec, $\left.\operatorname{sid}_{k_{0}}\right)=\operatorname{HeadTag}\left(\right.$ exec, $\left.u_{0}^{\prime \prime} \sigma\right)$, and thus ${\overline{\left.\left(u_{0}\right)\right|_{p}}}^{\text {exec,sid } \text { si }_{0}} \notin \mathcal{N}_{\epsilon}$.

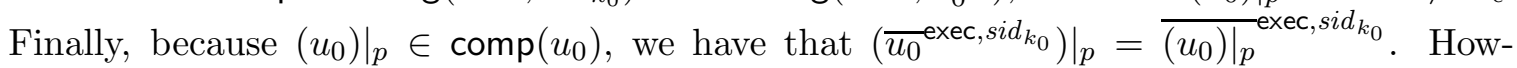
ever, this equality is not possible since we have shown that $\left.\left(\overline{u_{0}}{ }^{\text {exec, sid }} \bar{k}_{0}\right)\right|_{p} \in \mathcal{N}_{\epsilon}$ whereas ${\overline{\left.\left(u_{0}\right)\right|_{p}}}^{\text {exec, } s i d_{k_{0}}} \notin \mathcal{N}_{\epsilon}$. Hence, we obtain a contradiction.

Case 2: $\left.u_{0}\right|_{p} \in \bigcup_{x \in \operatorname{vars}\left(u_{0}^{\prime}\right)} \mathrm{St}_{\text {alien }}(\mathrm{exec}, x \sigma)$. In such a case, there exists $x \in \operatorname{vars}\left(u_{0}^{\prime}\right)$ such that $\left.u_{0}\right|_{p} \in \mathrm{St}_{\text {alien }}(\mathrm{exec}, x \sigma)$ and encs $\left(t_{1}, t_{2}\right) \in \mathrm{St}(x \sigma)$. Thanks to the origination property (see Definition 3.2 - Condition 19), we know that there exists $j<k_{0}$ such that ee $\operatorname{sid}_{j}=\operatorname{rcv}\left(v^{\prime}\right)$ and $x \in \operatorname{vars}\left(v^{\prime}\right)$. Hence, we have that encs $\left(t_{1}, t_{2}\right) \in \operatorname{St}\left(v^{\prime} \sigma\right)$. Since exec is a valid trace, we have that $\mathrm{K}\left(\operatorname{exec}_{j-1}\right) \cup T_{0} \vdash v^{\prime} \sigma$.

Let $\pi^{\prime}$ be a simple proof of $\mathrm{K}\left(\operatorname{exec}_{j-1}\right) \cup T_{0} \vdash v^{\prime} \sigma$, and $\pi^{\prime \prime}$ be a minimal subproof of $\pi^{\prime}$ whose root is labeled with a term $t^{\prime}$ such that encs $\left(t_{1}, t_{2}\right) \in \operatorname{St}\left(t^{\prime}\right)$. By locality of $\pi^{\prime}$ (Lemma 6.11), and because encs $\left(t_{1}, t_{2}\right) \notin \mathrm{St}\left(\mathrm{K}\left(\operatorname{exec}_{j-1}\right)\right.$ ) (remember here that we choose $k_{0}$ such that for all $j<k_{0}$, we have that encs $\left.\left(t_{1}, t_{2}\right) \notin \mathrm{St}\left(\mathrm{K}\left(\operatorname{exec}_{j-1}\right)\right)\right)$, we know that $\pi^{\prime \prime}$ ends with a composition rule. Unless $t^{\prime}=\operatorname{encs}\left(t_{1}, t_{2}\right)$, this contradicts the minimality of $\pi^{\prime \prime}$. Hence, we have that $t^{\prime}=\operatorname{encs}\left(t_{1}, t_{2}\right)$ and $\pi^{\prime \prime}$ is a simple proof of encs $\left(t_{1}, t_{2}\right)$ whose last rule is a composition. Actually, since encs $\left(t_{1}, t_{2}\right) \notin \mathrm{St}\left(\mathrm{K}\left(\operatorname{exec}_{j-1}\right) \cup T_{0}\right)$, any simple proof of encs $\left(t_{1}, t_{2}\right)$ ends with a composition. This will contradict the fact that $\pi$ is a simple proof of $t$.

This allows us to conclude the proof of the claim.

Now, by relying on our claim and by applying our induction hypothesis, we have that:

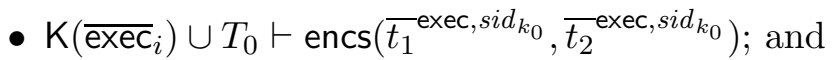

- $\mathrm{K}\left(\overline{\mathrm{exec}}_{i}\right) \cup T_{0} \vdash{\overline{t_{2}}}^{\mathrm{exec}, s^{2} d_{k_{0}}}$.

This allows us to deduce that $\mathrm{K}\left(\overline{\mathrm{exec}}_{i}\right) \cup T_{0} \vdash{\overline{t_{1}}}^{\mathrm{exec}, s_{i} d_{k_{0}}}$.

In order to establish that $\mathrm{K}\left(\overline{\operatorname{exec}}_{i}\right) \cup T_{0} \vdash \bar{t}^{\text {exec, sid }}$, we need to distinguish several cases:

Case $t \in \mathcal{A}, t=\operatorname{pub}(a)$ or $t=\mathrm{f}\left(a_{1}, \ldots, a_{n}\right)$ for some $\mathrm{f} \in\{$ shk, priv $\}$ :

In such a case, we have that $\bar{t}^{\mathrm{exec}, s i d}=\bar{t}^{\mathrm{exec}, s i d_{k_{0}}}=t$. Hence, we have that $\mathrm{K}\left(\overline{\operatorname{exec}}_{i}\right) \cup T_{0} \vdash$ $\bar{t}^{\text {exec,sid }}$ by applying some projection rules on the proof of $\mathrm{K}\left(\overline{\operatorname{exec}}_{i}\right) \cup T_{0} \vdash{\overline{t_{1}}}^{\text {exec, sid }}{ }_{k_{0}}$.

Case $t \in \mathcal{N}$ or $t=\mathrm{f}\left(t_{1}^{\prime}, \ldots, t_{m}^{\prime}\right)$ for some $\mathrm{f} \in\{$ encs, enca, $\mathrm{h}$, sign $\}$ :

If $\bar{t}^{\text {exec,sid }} \in \operatorname{comp}\left({\overline{t_{1}}}^{\text {exec,sid } k_{0}}\right)$, then we easily conclude that $\mathrm{K}\left(\overline{\operatorname{exec}}_{i}\right) \cup T_{0} \vdash \bar{t}^{\text {exec,sid }}$ since we have established that $\mathrm{K}\left(\overline{\operatorname{exec}}_{i}\right) \cup T_{0} \vdash{\overline{t_{1}}}^{\text {exec, sid } k_{0}}$. Otherwise, we have that $\bar{t}^{\text {exec, sid }} \notin \operatorname{comp}\left({\overline{t_{1}}}^{\text {exec, sid }} k_{0}\right)$. In that case, and according to Definition 6.6 and Lemma B.4 (item 11), either $\bar{t}^{\text {exec,sid }} \in \mathcal{N}_{\epsilon}$ or $\bar{t}^{\text {exec, sid }} k_{0} \in \mathcal{N}_{\epsilon}$. In the first case, we trivially conclude. In the second case, i.e. $\bar{t}^{\text {exec,sid }} \notin \mathcal{N}_{\epsilon}$ but $\bar{t}^{\text {exec,sid } k_{0}} \in \mathcal{N}_{\epsilon}$, we have that $t \in$ $\mathrm{St}_{\text {alien }}\left(\right.$ exec, encs $\left(t_{1}, t_{2}\right)$ ) (thanks to Lemma C.1. Since encs $\left(t_{1}, t_{2}\right) \in \operatorname{St}\left(u_{0}\right)$, we deduce that $t \in \mathrm{St}_{\text {alien }}\left(\mathrm{exec}, u_{0}\right) \cup \operatorname{comp}\left(\operatorname{encs}\left(t_{1}, t_{2}\right)\right)$ by applying Lemma B.5. Now, since $t \neq \operatorname{encs}\left(t_{1}, t_{2}\right)$, we deduce that $t \in \mathrm{St}_{\text {alien }}\left(\mathrm{exec}, u_{0}\right)$. Thus, applying Lemma B.7, we have 
that

$$
t \in \mathrm{St}_{\text {alien }}\left(\mathrm{exec}, u_{0}\right) \subseteq \operatorname{comp}\left(u_{0}\right) \cup \bigcup_{x \in \operatorname{vars}\left(u_{0}^{\prime}\right)} \mathrm{St}_{\mathrm{alien}}(\mathrm{exec}, \tau, x \sigma)
$$

where $\tau=$ ExpectedTag $\left(\right.$ exec, $\left.\operatorname{sid}_{k_{0}}\right)$.

Assume that $t \in \operatorname{comp}\left(u_{0}\right)$ and $t \notin \mathrm{St}_{\text {alien }}(\operatorname{exec}, \tau, x \sigma)$ for any $x \in \operatorname{vars}\left(u_{0}^{\prime}\right)$. In such a case, we have that there exists $t^{\prime} \in \operatorname{comp}\left(u_{0}^{\prime}\right)$ such that $t \in \operatorname{comp}\left(t^{\prime} \sigma\right)$ and we know that $t^{\prime} \notin \operatorname{vars}\left(u_{0}^{\prime}\right)$. Hence $t$ is either a nonce and we have that $t=t^{\prime}$. Moreover, we know that $t^{\prime}=n_{y}{ }^{s i d_{k_{0}}}$ for some $y$ (by construction of tr. In such a case, $\bar{t}^{\text {exec,sid }} k_{0} \notin \mathcal{N}_{\epsilon}$. This leads us to a contradiction. Otherwise $t$ is an encrypted term and we have that $t=t^{\prime} \sigma$ and again by construction of tr, we have that ${\overline{t^{\prime} \sigma}}^{\text {exec,sid } k_{0}} \notin \mathcal{N}_{\epsilon}$, leading us to a contradiction. Hence, we know that this case is not possible.

Hence, we have that $t \in \mathrm{St}_{\text {alien }}(\mathrm{exec}, \tau, x \sigma)$ for some $x \in \operatorname{vars}\left(u_{0}^{\prime}\right)$. Thanks to the origination property, we know that there exists $j<k_{0}$ such that $\operatorname{sid}_{j}=\operatorname{sid}_{k_{0}}$, ee sid $_{j}=$ $\operatorname{rcv}\left(v^{\prime}\right)$ with $x \in \operatorname{vars}\left(v^{\prime}\right)$. Hence, we have that $x \sigma \in \operatorname{St}\left(v^{\prime} \sigma\right)$. Then, applying LemmaB.8, we deduce that $t \in \mathrm{St}_{\text {alien }}$ (exec, $\tau, v^{\prime} \sigma$ ), and thanks to Lemma B.4 (item 2), we have that $t \in \mathrm{St}_{\text {alien }}\left(\mathrm{exec}, v^{\prime} \sigma\right)$.

Now, according to Lemma B.9, we know that $\mathrm{K}\left(\operatorname{exec}_{j-1}\right) \cup T_{0} \vdash w$ for any $w \in$ $\mathrm{St}_{\text {alien }}\left(\right.$ exec, $\left.\mathrm{K}\left(\operatorname{exec}_{j-1}\right) \cup T_{0}\right)$. Since exec is a valid trace, we have that $\mathrm{K}\left(\operatorname{exec}_{j-1}\right) \cup T_{0} \vdash$ $v^{\prime} \sigma$. Applying LemmaB.6, we deduce that $\mathrm{K}\left(\operatorname{exec}_{j-1}\right) \cup T_{0} \vdash w$ for any $w \in \mathrm{St}_{\text {alien }}$ (exec, $\left.v\right)$. In particular, we have that $\mathrm{K}\left(\operatorname{exec}_{j-1}\right) \cup T_{0} \vdash t$ and we conclude by relying on our induction hypothesis.

\section{Appendix D. Proofs of Section 6.4}

In order to prove Proposition 6.14 we will annotate formulas. For the sake of homogeneity, we chose to annotate each term that occurs in the formula even though it would have been sufficient to only annotate variables. Moreover, we state the definition for a general formula, but in our setting, terms that occur in a formula are either names or variables.

Definition D.1. (annotated formula) Given a formula $\phi$, we define its annotated version annotate $(\phi)$ as follows:

$$
\begin{array}{ll}
\text { annotate }(\text { true })=\operatorname{true} & \text { annotate }\left(\mathrm{Q}\left(t_{1}, \ldots, t_{n}\right)\right)=\mathrm{Q}\left(t_{1}^{t_{1}}, \ldots, t_{n}^{t_{n}}\right) \\
\text { annotate }(\neg \phi)=\neg \operatorname{annotate}(\phi) & \text { annotate }\left(\phi_{1} \vee \phi_{2}\right)=\operatorname{annotate}\left(\phi_{1}\right) \vee \text { annotate }\left(\phi_{2}\right) \\
\text { annotate }(\operatorname{learn}(t))=\operatorname{learn}\left(t^{t}\right) & \text { annotate }(\diamond \phi)=\diamond \operatorname{annotate}(\phi) \\
\operatorname{annotate}(\mathrm{C}(u))=\mathrm{C}\left(u^{u}\right) & \text { annotate }(\exists x . \phi)=\exists x \text {.annotate }(\phi)
\end{array}
$$

We emphasize that those annotations are syntactic decorations that do not interfere in the semantics of the formulas. We also suppose that these annotations are not affected by substitutions, i.e., when $x$ is a variable annotated with $a,\left(x^{a}\right) \sigma=(x \sigma)^{a}$. Relying on this notion of annotated formulas, we are now able to link each variable that occurs in $\phi$ with the term it has been substituted with in order to satisfy the formula. More precisely, we only need to know the session identifiers from which those terms are issued. The idea is that these sessions are important to satisfy the attack formula whereas the other ones could be discarded from the execution trace. 
Definition D.2. Let $\phi$ be an attack formula and $\psi$ its annotated version, i.e. $\psi=$ annotate $(\phi)$. Let $\Pi$ be a protocol, and exec $=\left[\mathrm{e}_{1}^{s i d_{1}} ; \ldots ; \mathrm{e}_{\ell}^{s i d_{\ell}}\right]$ be an execution trace (not necessarily valid) of $\Pi$ w.r.t. some initial intruder knowledge $T_{0}$ and such that $\left\langle\right.$ exec, $\left.T_{0}\right\rangle \models \psi$. Let $\pi$ be a proof tree witnessing the fact that $\left\langle\right.$ exec, $\left.T_{0}\right\rangle \models \psi$. We define $\mu(\pi)$ as described in Figure 2,

Intuitively, $\mu(\pi)$ maps variables occurring positively in a status event in the attack formula $\phi$ to session identifiers. Note also that since by definition of an attack formula each variable occurs at most once in a positive status event and by Condition 4 of Definition 4.3 , we have that $\mu(\pi)$ is actually a function.

Proposition 6.14. Let $\Pi$ be a protocol, exec be an execution trace of $\widetilde{\Pi}$ w.r.t. some initial intruder knowledge $T_{0}$, and $\phi$ be an attack formula. We have that

$$
\left\langle\text { exec, } T_{0}\right\rangle \models \phi \Rightarrow\left\langle\overline{\text { exec }}, T_{0}\right\rangle \models \phi \text {. }
$$

Proof. Let exec $=\left[\mathrm{e}_{1}^{s i d_{1}}, \ldots, \mathrm{e}_{\ell}^{\text {sid }} \ell\right]$ for some $\ell$, and some session identifiers $\operatorname{sid}_{1}, \ldots$, sid $_{\ell}$. By definition of an attack formula, $\phi$ is of the form

$$
\phi=\exists x_{1} \ldots \exists x_{n} . \psi
$$

for some quantifier-free formula $\psi$. Now, according to the semantics of $\mathcal{L},\left\langle\right.$ exec, $\left.T_{0}\right\rangle \models \phi$ implies that there exists $n$ ground terms $m_{1}, \ldots, m_{n}$ such that there exists a proof $\pi$ of $\left\langle\right.$ exec, $\left.T_{0}\right\rangle \models \phi$ of the form:

$$
\pi=\frac{\overline{\left\langle\operatorname{exec}, T_{0}\right\rangle \models \psi^{a} \sigma}}{\left\langle\operatorname{exec}, T_{0}\right\rangle \models \phi^{a}}
$$

where $\sigma=\left\{x_{1} \mapsto m_{1}, \ldots, x_{n} \mapsto m_{n}\right\}$ and $\phi^{a}=\exists x_{1} \ldots \exists x_{n} \cdot \psi^{a}=\operatorname{annotate}(\phi)$. Let $\bar{\sigma}=$ $\left\{x_{1} \mapsto{\overline{m_{1}}}^{\text {exec }, s i d_{1}^{\prime}}, \ldots, x_{n} \mapsto{\overline{m_{n}}}^{\text {exec, } s i d_{n}^{\prime}}\right\}$ where $\operatorname{sid}_{j}^{\prime}=\mu(\pi)\left(x_{j}\right)$ when $x_{j} \in \operatorname{dom}(\mu(\pi))$ and 0 otherwise.

Note that all except the last two nodes of $\pi$ are labeled with $\left\langle\operatorname{exec}_{i}, T_{0}\right\rangle \models \psi^{\prime} \sigma$ where $i \leq$ length (exec) and $\psi^{\prime}$ is smaller than $\psi$. Thus, the proof tree is finite. Moreover, by definition of $\mu$, we have that any leaf of $\pi$ of the form $\left\langle\operatorname{exec}_{i}, T_{0}\right\rangle \models \mathrm{Q}\left(u_{1}, \ldots, u_{k}\right) \sigma$ is such that $\mu(\pi)(x)=\operatorname{sid}_{i}$ for any $x \in \operatorname{vars}\left(\left\{u_{1}, \ldots, u_{k}\right\}\right)$. We prove that the proof tree obtained from $\pi$ by replacing each node labeled with $\left\langle\operatorname{exec}_{i}, T_{0}\right\rangle \models \psi^{\prime} \sigma$ by $\left\langle\operatorname{exec}_{i}, T_{0}\right\rangle \models \psi^{\prime} \bar{\sigma}$ is a (valid) proof tree witnessing the fact that $\left\langle\overline{\mathrm{exec}}, T_{0}\right\rangle \models \psi^{a} \bar{\sigma}$.

Base cases: the leaves of the proof tree $\pi$. In such a case, we have $\left\langle\operatorname{exec}_{i}, T_{0}\right\rangle \models \psi_{0} \sigma$ for a formula $\psi_{0}$ of the form true, $\mathrm{C}(x), \neg \mathrm{C}(x)$, learn $\left(u_{0}\right), \mathrm{Q}\left(u_{1}, \ldots, u_{k}\right)$, or $\neg Q\left(u_{1}, \ldots, u_{k}\right)$.

- $\psi_{0}=$ true: in such a case, we easily conclude.

- $\psi_{0}=\mathrm{C}(x)$ (resp. $\neg \mathrm{C}(x)$ ): in such a case, we have that $\mathrm{C}(x \sigma)=\mathrm{C}(x \bar{\sigma})$ since $\bar{a}^{\text {exec,sid }}=a$ for any agent name $a$ and any sid, and since the semantics of $C$ does not rely on the execution trace, we can also easily conclude in this case.

- $\psi_{0}=$ learn $\left(u_{0}\right)$ : in such a case, by definition of an attack formula, we know that $u_{0}$ is either an agent name (in such a case, we easily conclude) or a variable in $\left\{x_{1}, \ldots, x_{n}\right\}$. Let $j$ be such that $u_{0}=x_{j}$. By hypothesis, we have that $T_{0} \cup \mathrm{K}\left(\operatorname{exec}_{i}\right) \vdash u_{0} \sigma$. According to Lemma 6.12 , we know that $T_{0} \cup \mathrm{K}\left(\overline{\mathrm{exec}}_{i}\right) \vdash \overline{u_{0} \sigma}{ }^{\mathrm{exec}, \text { sid }}$ for any sid, and thus in particular

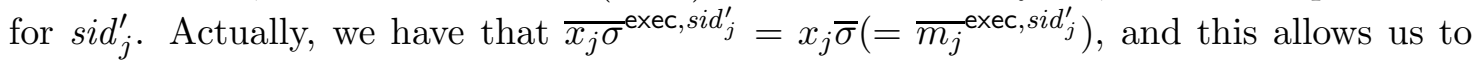
conclude that $\left\langle\overline{\operatorname{exec}}_{i}, T_{0}\right\rangle \models \operatorname{learn}\left(u_{0}\right) \bar{\sigma}$. 


$$
\begin{aligned}
& \mu\left(\overline{\left\langle\operatorname{exec}_{i}, T_{0}\right\rangle \models \text { true }}\right)=\emptyset \quad \mu\left(\overline{\left\langle\operatorname{exec}_{i}, T_{0}\right\rangle \models \operatorname{learn}\left(t^{u}\right)}\right)=\emptyset \\
& \mu\left(\overline{\left\langle\operatorname{exec}_{i}, T_{0}\right\rangle \models \mathrm{C}\left(u^{v}\right)}\right)=\emptyset \quad \mu\left(\overline{\left\langle\operatorname{exec}_{i}, T_{0}\right\rangle \models \neg \mathrm{C}\left(u^{v}\right)}\right)=\emptyset \\
& \mu\left(\overline{\left\langle\operatorname{exec}_{i}, T_{0}\right\rangle \models \neg \mathrm{Q}\left(t_{1}^{u_{1}}, \ldots, t_{n}^{u_{n}}\right)}\right)=\emptyset \\
& \mu\left(\overline{\left\langle\operatorname{exec}_{i}, T_{0}\right\rangle \models \mathrm{Q}\left(t_{1}^{u_{1}}, \ldots, t_{n}^{u_{n}}\right)}\right)=\underset{\text { where } \operatorname{vars}\left(\left\{u_{1}^{\prime}, \ldots, u_{n}\right\}\right)=\left\{u_{1}^{\prime}, \ldots, u_{m}^{\prime}\right\}}{\left.\left.\operatorname{sid} d_{i}\right)\right\}} \\
& \mu\left(\frac{\frac{\pi^{\prime}}{\left\langle\operatorname{exec}_{i}, T_{0}\right\rangle \models \psi_{j}}}{\left\langle\operatorname{exec}_{i}, T_{0}\right\rangle \models \psi_{1} \vee \psi_{2}}\right)=\mu\left(\frac{\pi^{\prime}}{\left\langle\operatorname{exec}_{i}, T_{0}\right\rangle \models \psi_{j}}\right) \text { with } j \in\{1,2\} \\
& \mu\left(\frac{\frac{\pi_{1}}{\left\langle\operatorname{exec}_{i}, T_{0}\right\rangle \models \neg \psi_{1}} \frac{\pi_{2}}{\left\langle\operatorname{exec}_{i}, T_{0}\right\rangle \models \neg \psi_{2}}}{\left\langle\operatorname{exec}_{i}, T_{0}\right\rangle \models \neg\left(\psi_{1} \vee \psi_{2}\right)}\right)=\bigcup_{j \in\{1,2\}} \mu\left(\frac{\pi_{j}}{\left\langle\operatorname{exec}_{i}, T_{0}\right\rangle \models \neg \psi_{j}}\right) \\
& \mu\left(\frac{\pi^{\prime}}{\frac{\left\langle\operatorname{exec}_{j}, T_{0}\right\rangle \models \psi}{\left\langle\operatorname{exec}_{i}, T_{0}\right\rangle \models \diamond \psi}}\right)=\mu\left(\frac{\pi^{\prime}}{\left\langle\operatorname{exec}_{j}, T_{0}\right\rangle \models \psi}\right) \text { where } j \leq i \\
& \mu\left(\frac{\frac{\pi_{1}}{\left\langle\operatorname{exec}_{1}, T_{0}\right\rangle \models \neg \psi} \ldots \frac{\pi_{i}}{\left\langle\operatorname{exec}_{i}, T_{0}\right\rangle \models \neg \psi}}{\left\langle\operatorname{exec}_{i}, T_{0}\right\rangle \models \neg(\diamond \psi)}\right)=\bigcup_{i \in\{1, \ldots, i\}} \mu\left(\frac{\pi_{i}}{\left\langle\operatorname{exec}_{i}, T_{0}\right\rangle \models \neg \psi}\right) \\
& \mu\left(\frac{\pi^{\prime}}{\left\langle\operatorname{exec}_{i}, T_{0}\right\rangle \models \psi\{t / x\}}\right)=\mu\left(\frac{\pi^{\prime}}{\left\langle\operatorname{exec}_{i}, T_{0}\right\rangle \models \exists x . \psi}\right) \\
& \mu\left(\frac{\frac{\pi^{\prime}}{\left\langle\operatorname{exec}_{i}, T_{0}\right\rangle \models \psi}}{\left\langle\operatorname{exec}_{i}, T_{0}\right\rangle \models \neg \neg \psi}\right)=\mu\left(\frac{\pi^{\prime}}{\left\langle\operatorname{exec}_{i}, T_{0}\right\rangle \models \psi}\right)
\end{aligned}
$$

Figure 2: Definition of the function $\mu$

- $\psi_{0}=\mathrm{Q}\left(u_{1}, \ldots, u_{k}\right)$ : in such a case, we know that each $u_{j}$ is either an agent name or a variable, and we have that $u_{j} \sigma=t_{j}$ for any $j \in\{1, \ldots, k\}$ where $\mathrm{e}_{i}^{\text {sidi }}=Q\left(t_{1}, \ldots, t_{k}\right)$. By definition of $\mu$, we have that either $u_{j}$ is an agent name or $u_{j}$ is a variable and $\mu(\pi)\left(u_{j}\right)=\operatorname{sid}_{i}$. In order to conclude that $\left\langle\overline{\operatorname{exec}}_{i}, T_{0}\right\rangle \models \mathrm{Q}\left(u_{1}, \ldots, u_{k}\right) \bar{\sigma}$, we have to show that ${\overline{t_{j}}}^{\text {exec,sid }}{ }=u_{j} \bar{\sigma}$. Let $j \in\{1, \ldots, k\}$. By hypothesis, we have that $u_{j} \sigma=t_{j}$, and 


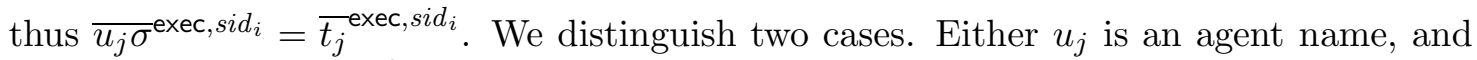
we have that $\overline{u_{j} \sigma^{\text {exec,sid }}}=u_{j}=u_{j} \bar{\sigma}$. Otherwise, $u_{j}$ is a variable, and we also have that ${\overline{u_{j}}}^{\text {exec, sid }} i_{i}=u_{j} \bar{\sigma}$ since by definition of $\mu$, we have that $\mu(\pi)\left(u_{j}\right)=\operatorname{sid}_{i}$.

- $\psi_{0}=\neg \mathrm{Q}\left(u_{1}, \ldots, u_{k}\right)$ : in such a case, we know that each $u_{j}$ is either an agent name or a variable, and we have that either $\operatorname{exec}_{i}=[]$ or $\mathrm{Q}\left(u_{1}, \ldots, u_{n}\right) \sigma \neq \mathrm{e}_{i}^{\text {sidi }_{i}}$. In the first case, we have that $\overline{\operatorname{exec}}_{i}=[]$ and we easily conclude. From now on, assume that $\mathrm{Q}\left(u_{1}, \ldots, u_{n}\right) \sigma \neq \mathrm{e}_{i}^{s i d_{i}}$. If $\mathrm{e}_{i}^{s i d_{i}} \neq \mathrm{Q}\left(t_{1}, \ldots, t_{k}\right)$ for any terms $t_{1}, \ldots, t_{k}$, then it is easy to

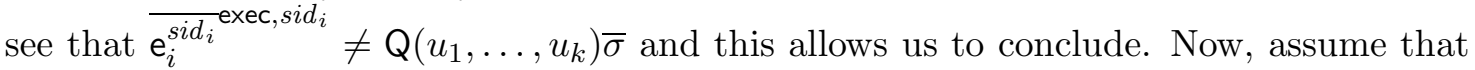
$\mathrm{e}_{i}^{s i d_{i}}=\mathrm{Q}\left(t_{1}, \ldots, t_{k}\right)$ for some terms $t_{1}, \ldots, t_{k}$. In such a case, there exists $j \in\{1, \ldots, k\}$ such that $u_{j} \sigma \neq t_{j}$. Using Lemma $\overline{C .2}$, we deduce that ${\overline{u_{j} \sigma}}^{\text {exec, } \mu\left(u_{j}\right)} \neq{\overline{t_{j}}}^{\text {exec, sid }}{ }^{\text {, and }}$

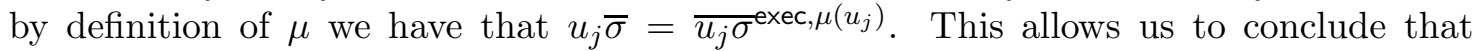
$\mathrm{Q}\left(u_{1}, \ldots, u_{k}\right) \bar{\sigma} \neq{\overline{\mathrm{Q}\left(t_{1}, \ldots, t_{k}\right)}}^{\mathrm{exec}, \text { sid }}$, and thus $\left\langle\overline{\operatorname{exec}}_{i}, T_{0}\right\rangle \models \psi_{0} \bar{\sigma}$.

Inductive cases. In such a case, we have that $\left\langle\operatorname{exec}_{i}, T_{0}\right\rangle \models \psi_{0} \sigma$ for a formula $\psi_{0}$ of the form $\neg \neg \psi_{0}^{\prime}, \psi_{1} \vee \psi_{2}, \neg\left(\psi_{1} \vee \psi_{2}\right), \diamond \psi_{0}^{\prime}$, or $\neg \nabla \psi_{0}^{\prime}$.

- $\psi_{0}=\neg \neg \psi_{0}^{\prime}$ : in such a case, we have that $\left\langle\operatorname{exec}_{i}, T_{0}\right\rangle \models \psi_{0}^{\prime} \sigma$, and using our induction hypothesis we conclude that $\left\langle\overline{\operatorname{exec}}_{i}, T_{0}\right\rangle \models \psi_{0}^{\prime} \bar{\sigma}$, and thus $\left\langle\overline{\operatorname{exec}}_{i}, T_{0}\right\rangle \models \neg \neg \psi_{0}^{\prime} \bar{\sigma}=\psi_{0} \bar{\sigma}$.

- $\psi_{0}=\psi_{1} \vee \psi_{2}$ : in such a case, we have that $\left\langle\operatorname{exec}_{i}, T_{0}\right\rangle \models \psi_{j} \sigma$ for some $j \in\{1,2\}$, and using our induction hypothesis we conclude that $\left\langle\overline{\operatorname{exec}}_{i}, T_{0}\right\rangle \models \psi_{j} \bar{\sigma}$, and thus $\left\langle\overline{\operatorname{exec}}_{i}, T_{0}\right\rangle \models$ $\left(\psi_{1} \vee \psi_{2}\right) \bar{\sigma}=\psi_{0} \bar{\sigma}$.

- $\psi_{0}=\neg\left(\psi_{1} \vee \psi_{2}\right)$ : in such a case, we have that $\left\langle\operatorname{exec}_{i}, T_{0}\right\rangle \models \neg \psi_{j}^{\prime} \sigma$ with $j \in\{1,2\}$, and using our induction hypothesis we conclude that $\left\langle\overline{\operatorname{exec}}_{i}, T_{0}\right\rangle \models \neg \psi_{j} \bar{\sigma}$ with $j \in\{1,2\}$, and thus $\left\langle\overline{\operatorname{exec}}_{i}, T_{0}\right\rangle \models \neg\left(\psi_{1} \vee \psi_{2}\right) \bar{\sigma}=\psi_{0} \bar{\sigma}$.

- $\psi_{0}=\diamond \psi_{0}^{\prime}$ : in such a case, we have that $\left\langle\operatorname{exec}_{j}, T_{0}\right\rangle \models \psi_{0}^{\prime} \sigma$ for some $j \leq i$, and using our induction hypothesis, we conclude that $\left\langle\overline{\operatorname{exec}_{j}}, T_{0}\right\rangle \models \psi_{0}^{\prime} \bar{\sigma}$, and thus $\left\langle\overline{\operatorname{exec}}_{i}, T_{0}\right\rangle \models \diamond \psi_{0}^{\prime} \bar{\sigma}=$ $\psi_{0} \bar{\sigma}$.

- $\psi_{0}=\neg \nabla \psi_{0}^{\prime}$ : in such a case, we have that $\left\langle\operatorname{exec}_{j}, T_{0}\right\rangle \models \neg \psi_{0}^{\prime} \sigma$ for any $j \in\{1, \ldots, j\}$, and using our induction hypothesis, we conclude that $\left\langle\overline{\operatorname{exec}_{j}}, T_{0}\right\rangle \models \neg \psi_{0}^{\prime} \bar{\sigma}$, and thus $\left\langle\overline{\operatorname{exec}}_{i}, T_{0}\right\rangle \models \neg \diamond \psi_{0}^{\prime} \bar{\sigma}=\psi_{0} \bar{\sigma}$.

\section{Appendix E. Proofs of Section 7}

This appendix contains the proofs of Section 7. Actually, Section E.1 contains the proofs related to the validity of the resulting trace exec $\left.\right|_{S}$ whereas SectionE.2 contains those related to the satisfiability of the attack formula.

E.1. Validity of the resulting trace. In order to preserve the validity of the resulting trace, it is important to show that sessions that are not tagged in the same way cannot share any name. This is the purpose of the following lemma. 
Lemma 7.2. Let $\Pi$ be a k-party protocol, and exec $=\left[\mathrm{e}_{1}^{s i d_{1}} ; \ldots ; \mathrm{e}_{\ell}^{\text {sid }}\right]$ be a well-formed valid execution of $\widetilde{\Pi}$ w.r.t. some set $T_{0}$ of ground atoms. Let sess 1 and sess $s_{2}$ be two session identifiers. We have that:

$$
\begin{aligned}
& \text { sameTagAs(exec, sess } 1) \neq \text { sameTagAs(exec, sess } 2) \\
& \text { implies } \\
& \text { names }(\text { exec, sess } 1) \cap \text { names }\left(\text { exec, sess } s_{2}\right)=\emptyset
\end{aligned}
$$

where names (exec, sess $)=\left\{u \mid u \in\right.$ names $\left(\mathrm{e}_{j}^{\text {sid }_{j}}\right)$ for some $1 \leq j \leq \ell$ such that sid $_{j}=$ sess $\}$.

Proof. Let sess $_{1}$ and sess $_{2}$ be two sessions and $n$ be a name such that:

- $\operatorname{sameTagAs}\left(\right.$ exec, sess $\left._{1}\right) \neq$ sameTagAs $\left(\right.$ exec, sess $\left._{2}\right)$; and

- $n \in$ names (exec, sess 1$) \cap$ names (exec, sess $_{2}$ ).

Let $S=$ sameTagAs(exec, sess 1 ). According to Condition 3 of well-formedness (Definition 6.4), $n \in$ names (exec, sess 1 ) implies that either $n$ is of the form $n_{t}^{\epsilon, S}$ or of the form $n_{t}^{\text {sid }}$ for some term $t$ and session identifier sid $\in S$. We treat these two cases separately:

Case $n=n_{t}^{\epsilon, S}$ : According to Condition 3 of well-formedness (Definition 6.4), we obtain $n_{t}^{\epsilon, S} \in$ names (exec, sess 2 ) implies that $S=$ sameTagAs (exec, sess 2$)$. But this contradicts the hypothesis sameTagAs(exec, sess 1$) \neq$ sameTagAs(exec, sess 2$)$.

Case $n=n_{t}^{\text {sid }}$ : In that case, sid $\in S$ and sameTagAs(exec, sid $)=\operatorname{sameTagAs}($ exec, sess 1$)$. Now, according to Condition 3 of well-formedness (Definition 6.4), we have that $n_{t}^{\text {sid }} \in$ names (exec, sess 2 ) implies that sid $\in$ sameTagAs(exec, sess 2$)$. However, this means that sameTagAs $($ exec, sid $)=$ sameTagAs $\left(\right.$ exec, sess $\left._{2}\right)$ which contradicts our hypothesis.

By contradiction we conclude that names (exec, sess 1$) \cap$ names $($ exec, sess 2$)=\emptyset$.

Now, provided that $S$ and $t$ satisfy some conditions, we show that a term $t$ that was deducible from exec will still be deducible from exec $\left.\right|_{S}$.

Lemma 7.3. Let $\Pi$ be a k-party protocol, and exec $=\left[\mathrm{e}_{1}^{s i d_{1}} ; \ldots ; \mathrm{e}_{\ell}^{\text {sid }}{ }_{\ell}\right]$ a well-formed valid execution of $\widetilde{\Pi}$ w.r.t. some set $T_{0}$ of ground atoms, and such that $T_{0} \cup \mathrm{K}(\mathrm{exec}) \forall k$ for any $k \in$ lgKeys $\backslash\left(\mathcal{K}_{\epsilon} \cup T_{0}\right)$ (exec does not reveal any long term keys). Let $S$ be a set of sessions such that:

for all session identifiers sess $s_{1}$ and sess $_{2}$ such that sameTagAs(exec, sess $\left._{1}\right)=$ sameTagAs(exec, sess $\left.s_{2}\right)$, we have that sess $s_{1} \in S$ if and only if sess $_{2} \in S$.

For all term $t \in \mathrm{St}\left(\left.\mathrm{exec}\right|_{S}\right)$ such that $T_{0} \cup \mathrm{K}(\mathrm{exec}) \vdash t$, we have that $T_{0} \cup \mathrm{K}\left(\left.\operatorname{exec}\right|_{S}\right) \vdash t$.

Proof. Let sid $\in S, t \in \mathrm{St}(\mathrm{exec}$, sid $)$, and $\pi$ be a simple proof of $T_{0} \cup \mathrm{K}(\mathrm{exec}) \vdash t$. We prove this result by structural induction on $\pi$. But, we first need to establish the following preliminary result (still under the hypotheses stated in Lemma 7.3).

Claim. If names $(t) \subseteq \mathcal{N}_{\epsilon}$ then $T_{0} \vdash t$.

Proof of the claim. Let us suppose that there exists $u \in \operatorname{CryptSt}(t)$. Because exec is wellformed, we know by Conditions 1 and 2 of well-formedness (Definition 6.4) that $t$ is $k$-tagged and thus that $u=\mathrm{f}\left(\left\langle\tau, u_{1}\right\rangle, \ldots, u_{n}\right)$ with $\tau=$ ExpectedTag(exec, sid $) \neq \perp$. Now, according to the definition of a symbolic trace (Definition 3.5) and of our protocol transformation (Definition [5.2), we know that there exists $n_{v}^{\text {sid }} \in$ names $(\tau) \subseteq$ names $(u) \subseteq$ names $(t)$, which contradicts the hypothesis that names $(t) \subseteq \mathcal{N}_{\epsilon}$. Thus it must be that $\operatorname{CryptSt}(t)=\emptyset$, and 
hence, $t$ must be a tuple of atoms, i.e. a tuple of terms in $\mathcal{A} \cup T_{0} \cup \mathcal{N}_{\epsilon} \cup \mathcal{K}_{\epsilon} \cup\{\operatorname{pub}(a)$ $a \in \mathcal{A}\} \cup\{\operatorname{priv}(a), \operatorname{shk}(a, b) \mid a, b \in \mathcal{A}\}$. Now, because we only consider executions that do not reveal any long-term decryption keys, we necessarily have that the atomic subterms of $t$ are in $\mathcal{A} \cup T_{0} \cup \mathcal{N}_{\epsilon} \cup \mathcal{K}_{\epsilon} \cup\{\operatorname{pub}(a) \mid a \in \mathcal{A}\}$. This implies according to Definition 2.2, that any atomic subterm of $t$ is deducible from $T_{0}$. Finally, since $t$ is a tuple of deducible terms, $t$ can be deduced by application of the pairing rule, and thus $T_{0} \vdash t$.

We now proceed with our induction

Base case: $\pi$ is reduced to a leaf: In that case, $t \in \mathcal{A} \cup T_{0} \cup \mathcal{N}_{\epsilon} \cup \mathcal{K}_{\epsilon} \cup\{\operatorname{pub}(a) \mid a \in$ $\mathcal{A}\} \cup \mathrm{K}($ exec $)$. If names $(t) \subseteq \mathcal{N}_{\epsilon}$, then by the above claim we have that $T_{0} \vdash t$, and thus $T_{0} \cup \mathrm{K}\left(\left.\operatorname{exec}\right|_{S}\right) \vdash t$. Let us now suppose that there exists $n_{v}^{s i d^{\prime}} \notin \mathcal{N}_{\epsilon}$. In that case, $n_{v}^{\text {sid }} \in$ names (exec, sid) and $t \in \mathrm{K}(\mathrm{exec})$, i.e. there exists $i \in\{1, \ldots, \ell\}$, such that $\mathrm{e}_{i}^{s i d_{i}}=\operatorname{snd}(t)$. Thus, $n_{v}^{\text {sid }} \in$ names (exec, sid $\left._{i}\right)$ and hence names (exec, sid $) \cap$ names (exec, sid $\left._{i}\right) \neq \emptyset$. This, according to Lemma 7.2, implies that

$$
\text { sameTagAs }\left(\text { exec, } \text { sid }_{i}\right)=\text { sameTagAs }(\text { exec, } \text { sid })
$$

By hypothesis on $S$, we have $\operatorname{sid}_{i} \in S$, and by definition we have $\left.\mathrm{e}_{i}^{\text {sid }_{i}} \in \operatorname{exec}\right|_{S}$, which implies that $t \in \mathrm{K}\left(\left.\operatorname{exec}\right|_{S}\right)$. We can thus conclude that $T_{0} \cup \mathrm{K}\left(\left.\operatorname{exec}\right|_{S}\right) \vdash t$.

Inductive case: In that case we need to distinguish two cases according to the last rule applied in the proof $\pi$.

Case 1 - the last rule is a composition rule: We have that the term $t$ is of the form $\mathrm{f}\left(t_{1}, \ldots, t_{n}\right)$, and the derivation $T_{0} \cup \mathrm{K}($ exec $) \vdash t$ is of the form

$$
\frac{T_{0} \cup \mathrm{K}(\text { exec }) \vdash t_{1} \quad \ldots \quad T_{0} \cup \mathrm{K}(\text { exec }) \vdash t_{n}}{T_{0} \cup \mathrm{K}(\text { exec }) \vdash \mathrm{f}\left(t_{1}, \ldots, t_{n}\right)}
$$

For all $i \in\{1, \ldots, n\}, t_{i} \in \mathrm{St}(t) \subseteq \mathrm{St}($ exec, sid $)$, and by induction hypothesis $T_{0} \cup$ $\mathrm{K}\left(\left.\operatorname{exec}\right|_{S}\right) \vdash t_{i}$. We can thus conclude that by application of the corresponding composition rule. We have that:

$$
\frac{T_{0} \cup \mathrm{K}\left(\left.\mathrm{exec}\right|_{S}\right) \vdash t_{1} \quad \ldots \quad T_{0} \cup \mathrm{K}\left(\left.\operatorname{exec}\right|_{S}\right) \vdash t_{n}}{T_{0} \cup \mathrm{K}\left(\left.\operatorname{exec}\right|_{S}\right) \vdash f\left(t_{1}, \ldots, t_{n}\right)}
$$

Case 2 - the last rule is a decomposition rule: We have that the proof tree witnessing $T_{0} \cup \mathrm{K}($ exec $) \vdash t$ is of the form

$$
\frac{T_{0} \cup \mathrm{K}(\text { exec }) \vdash t_{1} \quad \ldots \quad T_{0} \cup \mathrm{K}(\text { exec }) \vdash t_{n}}{T_{0} \cup \mathrm{K}(\text { exec }) \vdash t}
$$

If names $(t) \subseteq \mathcal{N}_{\epsilon}$, then we have seen that $T_{0} \vdash t$, and thus we conclude. Now, assume that there exists $n_{v}^{\text {sid }} \in\left(\right.$ names $\left.(t) \backslash \mathcal{N}_{\epsilon}\right) \subseteq$ names (exec, sid). By Definition of a symbolic trace and of an execution trace (see Definition 3.5), $n_{v}^{\text {sid }} \in$ names (exec, sid'). Thus names (exec, sid) $\cap$ names (exec, sid $) \neq \emptyset$, and thanks to Lemma 7.2, we have that: sameTagAs (exec, sid $)=$ sameTagAs (exec, sid ${ }^{\prime}$.

We need to prove that for all $i \in\{1, \ldots, n\}, T_{0} \cup \mathrm{K}\left(\left.\operatorname{exec}\right|_{S}\right) \vdash t_{i}$. Since $\pi$ is minimal, we know by locality (Lemma 6.11) that $t_{i} \in \mathrm{St}\left(T_{0} \cup \mathcal{N}_{\epsilon} \cup \mathcal{K}_{\epsilon} \cup \mathrm{K}(\operatorname{exec})\right) \cup \mathcal{A} \cup\{\operatorname{pub}(a) \mid a \in$ $\mathcal{A}\}$. We consider two cases:

If names $\left(t_{i}\right) \subseteq \mathcal{N}_{\epsilon}$, then we have already established that $T_{0} \vdash t_{i}$, and thus $T_{0} \cup$ $\mathrm{K}\left(\left.\operatorname{exec}\right|_{S}\right) \vdash t_{i}$. 
Otherwise, there exists $n_{w}^{s i d^{\prime \prime}} \in\left(\right.$ names $\left.\left(t_{i}\right) \backslash \mathcal{N}_{\epsilon}\right)$. In that case, $t_{i} \in \mathrm{St}(\mathrm{K}(\mathrm{exec}))$, i.e. there exists $k \in\{1, \ldots, \ell\}$ such that $t_{i} \in \mathrm{St}\left(\mathrm{e}_{k}^{\text {sid }_{k}}\right) \subseteq \mathrm{St}\left(\mathrm{exec}, \operatorname{sid}_{k}\right)$; and thus $n_{w}^{\text {sid }} \in$ names $\left(\mathrm{exec}, \operatorname{sid}_{k}\right)$. Moreover, by Definition of a symbolic trace and of an execution trace (see Definition 3.5),$n_{w}^{\text {sid }} \in$ names (exec, sid" $)$. Hence, we have that names $\left(\mathrm{exec}\right.$, sid $\left.^{\prime \prime}\right) \cap$ names $\left(\mathrm{exec}, \operatorname{sid}_{k}\right) \neq \emptyset$, which according to Lemma 7.2 implies that

$$
\text { sameTagAs }\left(\text { exec, sid") }=\text { sameTagAs }\left(\text { exec, } \operatorname{sid}_{k}\right)\right. \text {. }
$$

By inspection of the decomposition rules, we note that there must exist $j \in\{1, \ldots, n\}$, such that for all $i \in\{1, \ldots, n\}$, names $(t) \cup \operatorname{names}\left(t_{i}\right) \subseteq$ names $\left(t_{j}\right)$, and therefore $n_{v}^{s i d^{\prime}}, n_{w}^{s i d^{\prime \prime}} \in\left(\right.$ names $\left.\left(t_{j}\right) \backslash \mathcal{N}_{\epsilon}\right)$. Moreover, we have that $t_{j} \in \mathrm{St}(\mathrm{K}(\mathrm{exec}))$, i.e. there exists $h \in\{1, \ldots, \ell\}$ such that $t_{j} \in \mathrm{St}\left(\mathrm{e}_{j}^{s i d_{h}}\right) \subseteq \mathrm{St}\left(\mathrm{exec}, \operatorname{sid}_{h}\right)$. Hence, $n_{v}^{s i d^{\prime}}, n_{w}^{s i d^{\prime \prime}} \in$ names (exec, sid $_{h}$ ), which according to Lemma 7.2 implies

$$
\begin{aligned}
& \text { sameTagAs }\left(\text { exec, } \text { sid }^{\prime}\right)=\text { sameTagAs }\left(\text { exec, } \text { sid }_{h}\right) \\
& \text { sameTagAs }\left(\text { exec, sid } \text { sin }^{\prime \prime}\right)=\operatorname{sameTagAs}\left(\text { exec, } \text { sid }_{h}\right)
\end{aligned}
$$

We therefore can infer that

$$
\text { sameTagAs }(\text { exec, sid })=\text { sameTagAs }\left(\text { exec, } \operatorname{sid}_{k}\right) \text {. }
$$

and by hypothesis on $S$ that $\operatorname{sid}_{k} \in S$. We have thus demonstrated that $t_{i} \in$ St $\left(\right.$ exec, $\left.\operatorname{sid}_{k}\right)$ with $\operatorname{sid}_{k} \in S$, which according to our induction hypothesis implies $T_{0} \cup \mathrm{K}\left(\left.\mathrm{exec}\right|_{S}\right) \vdash t_{i}$.

Since for all $i \in\{1, \ldots, n\}, T_{0} \cup \mathrm{K}\left(\left.\operatorname{exec}\right|_{S}\right) \vdash t_{i}$, we can conclude by application of the corresponding decomposition rule that:

$$
\frac{T_{0} \cup \mathrm{K}\left(\left.\operatorname{exec}\right|_{S}\right) \vdash t_{1} \quad \ldots \quad T_{0} \cup \mathrm{K}\left(\left.\operatorname{exec}\right|_{S}\right) \vdash t_{n}}{T_{0} \cup \mathrm{K}\left(\left.\operatorname{exec}\right|_{S}\right) \vdash t}
$$

\section{E.2. Satisfiability of the formula.}

Lemma E.1. Let $\Pi$ be a $k$-party protocol, $\phi$ a closed quantifier-free formula in $\mathcal{L}$, and exec $=\left[\mathrm{e}_{1}^{s i d_{1}} ; \ldots ; \mathrm{e}_{\ell}^{\text {sid }}\right]$ be a well-formed valid execution of $\widetilde{\Pi}$ that satisfies $\phi$, w.r.t. some set $T_{0}$ of ground atoms. Moreover, we assume that $T_{0} \cup \mathrm{K}(\mathrm{exec}) \forall k$ for any $k \in \operatorname{lgKeys} \backslash\left(\mathcal{K}_{\epsilon} \cup T_{0}\right)$ (exec does not reveal any long term keys). Let $S$ be a set of session identifiers such that:

(1) for all learn $(t)$ that occurs positively in $\phi$ such that $t \notin \mathcal{A} \cup$ lgKeys, there exists sid $\in S$ such that $t \in \mathrm{St}(\mathrm{exec}$, sid $)$,

(2) $\mathrm{Ws}($ exec, $\phi) \subseteq S$, and

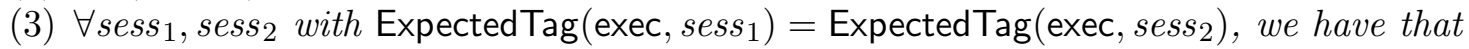
sess $_{1} \in S$ if and only if sess $s_{2} \in S$.

We have that exec $\left.\right|_{S}$ is an execution of $\widetilde{\Pi}$ that satisfies $\phi$, i.e. $\left\langle\left.\operatorname{exec}\right|_{S}, T_{0}\right\rangle \models \phi$.

Proof. We prove this by induction on $(\ell$, size $(\phi))$ using the lexicographic ordering. Here, $\ell$ denotes the length (i.e. number of events) of the trace exec and size $(\phi)$ is the size of $\phi$ (i.e. number of symbols that occur in $\phi$ without counting the symbol $\neg$ and after elimination of double negation, i.e., $\neg \neg \psi$ is rewritten in $\psi$ ).

We need to distinguish several base cases. 
Case $\mid$ exec $\mid=0$ : In that case exec $\left.\right|_{S}=$ exec, and thus by hypothesis if $\left\langle\right.$ exec, $\left.T_{0}\right\rangle \models \phi$, then also $\left\langle\right.$ exec $\left.\left.\right|_{S}, T_{0}\right\rangle \models \phi$.

Case $\phi=$ true (resp. $\phi=\neg$ true): In such a case, we have that $\left\langle\right.$ exec $\left.\left.\right|_{S}, T_{0}\right\rangle \models \phi$. The case where $\phi=\neg$ true is impossible.

Case $\phi=\mathrm{Q}\left(t_{1}, \ldots, t_{n}\right)$ : If $\left\langle\right.$ exec, $\left.T_{0}\right\rangle \models \phi$, then $\mathrm{e}_{\ell}^{\text {sid }}=\mathrm{Q}\left(t_{1}, \ldots, t_{n}\right)$, and $\mathrm{Ws}(\mathrm{exec}, \phi)=$ $\left\{\operatorname{sid}_{\ell}\right\} \subseteq S$. By Definition [7.1, exec| $\left.\right|_{S}$ ends with the event $\mathrm{Q}\left(t_{1}, \ldots, t_{n}\right)$. We can thus conclude that $\left\langle\left.\operatorname{exec}\right|_{S}, T_{0}\right\rangle \models \phi$.

Case $\phi=\neg \mathrm{Q}\left(t_{1}, \ldots, t_{n}\right)$ : If $\left\langle\right.$ exec, $\left.T_{0}\right\rangle \models \neg \mathrm{Q}\left(t_{1}, \ldots, t_{n}\right)$, we have that $\mathrm{e}_{\ell}^{\text {sid }} \neq \mathrm{Q}\left(t_{1}, \ldots, t_{n}\right)$, and $\mathrm{Ws}($ exec, $\phi)=\left\{\operatorname{sid}_{\ell}\right\} \subseteq S$ (note that we have already considered the case where exec $=[]$, and thus now we assume that exec $\neq[])$. We have that exec $\left.\right|_{S}$ does not end with $\mathrm{Q}\left(t_{1}, \ldots, t_{n}\right)$. We can thus conclude that $\left\langle\left.\operatorname{exec}\right|_{S}, T_{0}\right\rangle \models \neg \mathrm{Q}\left(t_{1}, \ldots, t_{n}\right)$, i.e. $\left\langle\left.\operatorname{exec}\right|_{S}, T_{0}\right\rangle \models \phi$.

Case $\phi=\operatorname{learn}(t)$ : If $\left\langle\right.$ exec, $\left.T_{0}\right\rangle \models \phi$, then $T_{0} \cup \mathrm{K}($ exec $) \vdash t$. If $t \in \mathcal{A} \cup \lg K e y s$, since exec doesn't reveal any long-term decryption key, $T_{0} \vdash t$, and thus $T_{0} \cup \mathrm{K}\left(\left.\operatorname{exec}\right|_{S}\right) \vdash t$. If $t \notin$ $\mathcal{A} \cup$ lgKeys, then by hypothesis we know there exists sid $\in S$ such that $t \in \mathrm{St}(\mathrm{exec}$, sid). According to Lemma 7.3, since by hypothesis sid $\in S, T_{0} \cup \mathrm{K}\left(\left.\operatorname{exec}\right|_{S}\right) \vdash t$. Hence, we can conclude that $\left\langle\left.\operatorname{exec}\right|_{S}, T_{0}\right\rangle \models \phi$.

Case $\phi=\neg$ learn $(t)$ : If $\left\langle\right.$ exec, $\left.T_{0}\right\rangle \models \neg$ learn $(t)$, then $T_{0} \cup \mathrm{K}($ exec $) \forall t$. But since $T_{0} \cup$ $\mathrm{K}\left(\left.\operatorname{exec}\right|_{S}\right) \subseteq T_{0} \cup \mathrm{K}($ exec $)$, it is also the case that $T_{0} \cup \mathrm{K}\left(\right.$ exec $\left.\left.\right|_{S}\right) \forall t$, and thus that $\left\langle\left.\operatorname{exec}\right|_{S}, T_{0}\right\rangle \models \neg$ learn $(t)$.

Case $\phi=\mathrm{C}(u)$ : If $\left\langle\right.$ exec, $\left.T_{0}\right\rangle \models \mathrm{C}(u)$, then we have that $T_{0} \vdash \operatorname{priv}(u)$ or $T_{0} \vdash \operatorname{shk}(u, v)$ for some $v \neq \epsilon$. Hence, we also have that $\left\langle\left.\operatorname{exec}\right|_{S}, T_{0}\right\rangle \models \mathrm{C}(t)$.

Case $\phi=\neg \mathrm{C}(u)$ : If $\left\langle\operatorname{exec}, T_{0}\right\rangle \models \neg \mathrm{C}(u)$, then we have that $T_{0} \not \forall \operatorname{priv}(u)$ and $T_{0} \not \forall \operatorname{shk}(u, v)$ for all $v \neq \epsilon$. Hence, we also have that $\left\langle\left.\operatorname{exec}\right|_{S}, T_{0}\right\rangle \models \neg \mathrm{C}(t)$.

We distinguish several inductive cases $(|\operatorname{exec}|>1$ and $\operatorname{size}(\phi)>1)$.

Case $\phi=\phi_{1} \vee \phi_{2}$ : If $\left\langle\right.$ exec, $\left.T_{0}\right\rangle \models \phi$ then $\left\langle\right.$ exec, $\left.T_{0}\right\rangle \models \phi_{1}$ or else $\left\langle\right.$ exec, $\left.T_{0}\right\rangle \models \phi_{2}$. Assume that $\left\langle\right.$ exec, $\left.T_{0}\right\rangle \models \phi_{1}$ (the other case can be done in a similar way). It is easy to see that the three conditions needed to apply our inductive hypothesis are fulfilled. We can thus apply our inductive hypothesis on $\phi_{1}$ to conclude that $\left\langle\left.\operatorname{exec}\right|_{S}, T_{0}\right\rangle \models \phi_{1}$, and thus that $\left\langle\right.$ exec $\left.\left.\right|_{S}, T_{0}\right\rangle \models \phi_{1} \vee \phi_{2}$.

Case $\phi=\neg\left(\phi_{1} \vee \phi_{2}\right)$ : If $\left\langle\right.$ exec, $\left.T_{0}\right\rangle \models \neg\left(\phi_{1} \vee \phi_{2}\right)$, then $\left\langle\right.$ exec, $\left.T_{0}\right\rangle \models \neg \phi_{1}$ and $\left\langle\right.$ exec, $\left.T_{0}\right\rangle \models$ $\neg \phi_{2}$. Again, the three conditions needed to apply our inductive hypothesis are full-filled. We can thus apply our inductive hypothesis to conclude that $\left\langle\left.\operatorname{exec}\right|_{S}, T_{0}\right\rangle \models \neg \phi_{1}$ and $\left\langle\left.\operatorname{exec}\right|_{S}, T_{0}\right\rangle \models \neg \phi_{2}$, and thus $\left\langle\left.\operatorname{exec}\right|_{S}, T_{0}\right\rangle \models \neg\left(\phi_{1} \vee \phi_{2}\right)$.

Case $\phi=\diamond \psi$ : If $\left\langle\right.$ exec, $\left.T_{0}\right\rangle \models \phi$, then we know that there exists $i \in\{1, \ldots, \ell\}$ such that $\left\langle\operatorname{exec}_{i}, T_{0}\right\rangle \models \psi$ and $\mathrm{Ws}(\operatorname{exec}, \phi)=\mathrm{Ws}\left(\operatorname{exec}_{i}, \psi\right)$.

- Let learn $(t)$ be a subformula that occurs positively in $\psi$ such that $t \notin \mathcal{A} \cup$ lgKeys. Then, by definition, learn $(t)$ also occurs positively in $\phi$, and thus by hypothesis, there exists sid $\in S$ such that $t \in \mathrm{St}(\mathrm{exec}$, sid).

- We have that Ws $($ exec, $\phi)=\mathrm{Ws}_{\mathbf{s}}\left(\operatorname{exec}_{i}, \psi\right)$, and by hypothesis $\mathrm{Ws}(\operatorname{exec}, \phi) \subseteq S$. Thus $\mathrm{Ws}\left(\operatorname{exec}_{i}, \psi\right) \subseteq S$.

- By hypothesis, $S$ satisfies: for all $s e s s_{1}$ and $s e s s_{2}$ with ExpectedTag(exec, sess 1$)=$ ExpectedTag (exec, sess 2 ), sess $1 \in S$ if and only if $\operatorname{sess}_{2} \in S$.

The three conditions are fulfilled, we can thus apply our inductive hypothesis to conclude that $\left.\operatorname{exec}_{i}\right|_{S}$ also satisfies $\psi$, i.e. $\left\langle\left.\operatorname{exec}_{i}\right|_{S}, T_{0}\right\rangle \models \psi$. But then there exists $j$ such that 
$\left.\operatorname{exec}_{i}\right|_{S}=\left(\left.\operatorname{exec}\right|_{S}\right)_{j}$, and thus such that $\left\langle\left(\left.\operatorname{exec}\right|_{S}\right)_{j}, T_{0}\right\rangle \models \psi$, which according to the semantics of $\mathcal{L}$ gives us exec $\left.\right|_{S}$ satisfies $\diamond \psi$, i.e. $\left\langle\left.\operatorname{exec}\right|_{S}, T_{0}\right\rangle \models \diamond \psi$.

Case $\phi=\neg \nabla \psi$ : If $\left\langle\right.$ exec, $\left.T_{0}\right\rangle \models \neg \nabla \psi$, then according to the semantics of $\mathcal{L}$, we have that $\left\langle\operatorname{exec}_{\ell-1}, T_{0}\right\rangle \models \neg \nabla \psi$ and $\left\langle\right.$ exec, $\left.T_{0}\right\rangle \models \neg \psi$.

- In the syntax of $\mathcal{L}$, see Definition 4.1, learn $(t)$ must not occur under a modality, so the first condition is trivially fulfilled.

- By definition, Ws $($ exec, $\phi)=\emptyset \subseteq S$.

- By hypothesis, $S$ satisfies: for all sess $_{1}$ and $s e s s_{2}$ with ExpectedTag (exec, sess 1$)=$ ExpectedTag (exec, sess 2$),$ sess $_{1} \in S$ if and only if sess $_{2} \in S$.

We apply our inductive hypothesis and conclude that $\left\langle\left.\left(\operatorname{exec}_{\ell-1}\right)\right|_{S}, T_{0}\right\rangle \models \neg \nabla \psi$. Now, we distinguish two cases: either $\operatorname{sid}_{\ell} \in S$ or sid $_{\ell} \notin S$. In the first case, we can also apply our inductive hypothesis on $\left\langle\right.$ exec, $\left.T_{0}\right\rangle \models \neg \psi$ (note that $\mathrm{Ws}(\mathrm{exec}, \psi) \subseteq\left\{\operatorname{sid}_{\ell}\right\} \subseteq S$ since $\psi$ is from the restricted syntax according to Definition 4.1) and conclude that $\left\langle\left.\operatorname{exec}\right|_{S}, T_{0}\right\rangle \models \neg \psi$. This allows us to conclude that $\left\langle\left.\operatorname{exec}\right|_{S}, T_{0}\right\rangle \models \neg \diamond \psi$. In the second case, we have that exec $\left.\right|_{S}=\left.\operatorname{exec}_{\ell-1}\right|_{S}$, and thus conclude that $\left\langle\left.\operatorname{exec}\right|_{S}, T_{0}\right\rangle \models \neg \diamond \psi$.

Lemma E.2. Let $\Pi$ be a k-party protocol, exec $\left.=\left[\mathrm{e}_{1}^{\text {sid }}{ }_{1} ; \ldots ; \mathrm{e}_{\ell}^{\text {sid }}\right]_{\ell}\right]$ be a valid execution of $\widetilde{\Pi}$ w.r.t. some set $T_{0}$ of ground atoms, $\phi=\exists x_{1} \ldots \exists x_{n} . \psi$ be an attack formula of $\mathcal{L}$ (see Definition 4.3), $\sigma=\left\{x_{1} \mapsto m_{1}, \ldots, x_{n} \mapsto m_{n}\right\}$ be a ground substitution, $S$ be a set of session identifiers such that $\mathrm{Ws}(\mathrm{exec}, \psi \sigma) \subseteq S$, and $n_{\epsilon}^{\epsilon} \in \mathcal{N}_{\epsilon}$ be an intruder nonce not appearing in exec. If $\left\langle\right.$ exec, $\left.T_{0}\right\rangle \models \psi \sigma$ then we have that $\left\langle\right.$ exec, $\left.T_{0}\right\rangle \models \psi \sigma^{\prime}$ where for all $j \in\{1, \ldots, n\}$

$$
\sigma^{\prime}\left(x_{j}\right)=\left\{\begin{array}{lr}
n_{\epsilon}^{\epsilon} & \text { if } \sigma\left(x_{j}\right) \notin \mathrm{St}(\text { exec, } S) \cup \mathcal{A} \cup \operatorname{lgKeys} \cup \mathcal{N}_{\epsilon} \cup \mathcal{K}_{\epsilon} \\
\sigma\left(x_{j}\right) & \text { otherwise }
\end{array}\right.
$$

Proof. We prove this result by induction on $(\ell$, size $(\psi))$ using the lexicographic ordering where $\ell$ denotes the length of the trace exec, and size $(\psi)$ the size of $\psi$ (i.e. number of symbols that occur in $\psi$ without counting the symbol $\neg$ and after elimination of double negation, i.e., $\neg \neg \psi$ is rewritten in $\psi$ ). Actually, we strengthen the induction hypothesis by only requiring the hypothesis $\mathrm{Ws}\left(\operatorname{exec}_{p}, \psi \sigma\right) \subseteq S$ when some status event occurs positively in $\psi \sigma$.

Base case $(\operatorname{size}(\psi)=1)$ : We distinguish several base cases.

- Case $\psi^{\prime}=$ true. In that case $\psi \sigma=\psi \sigma^{\prime}=$ true and we easily conclude.

- Case $\psi^{\prime}=\neg$ true. This case is impossible since such a formula is not satisfiable.

- Case $\psi=\mathrm{Q}\left(t_{1}, \ldots, t_{h}\right)$. In that case, we have that $\psi \sigma=\mathrm{Q}\left(t_{1} \sigma, \ldots, t_{h} \sigma\right)$, and $\mathrm{e}_{p}^{\text {sid }_{p}}=$ $\mathrm{Q}\left(t_{1} \sigma, \ldots, t_{h} \sigma\right)$ with $\operatorname{sid}_{p} \in S$. But then, by Definition of $\sigma^{\prime}$, we have that $\mathrm{e}_{p}^{s i d_{p}}=$ $\mathrm{Q}\left(t_{1} \sigma, \ldots, t_{h} \sigma\right)=\mathrm{Q}\left(t_{1} \sigma^{\prime}, \ldots, t_{h} \sigma^{\prime}\right)$, and we conclude that $\left\langle\operatorname{exec}_{p}, T_{o}\right\rangle \models \psi \sigma^{\prime}$.

- Case $\psi=\neg \mathrm{Q}\left(t_{1}, \ldots, t_{h}\right)$. In that case, $\psi \sigma=\mathrm{Q}\left(t_{1} \sigma, \ldots, t_{h} \sigma\right)$, and either $\operatorname{exec}_{p}=[]$ or $\mathrm{e}_{p}^{\text {sid }} \neq \mathrm{Q}\left(t_{1} \sigma, \ldots, t_{h} \sigma\right)$. In the first case, according to the semantics of our logic $\mathcal{L}$, we conclude that $\left\langle\operatorname{exec}_{p}, T_{0}\right\rangle \models \psi \sigma^{\prime}\left(=\neg \mathrm{Q}\left(t_{1} \sigma^{\prime}, \ldots, t_{h} \sigma^{\prime}\right)\right)$. In the second case, i.e. $\mathrm{e}_{p}^{s i d_{p}} \neq \mathrm{Q}\left(t_{1} \sigma, \ldots, t_{h} \sigma\right)$, by Definition of $\sigma^{\prime}$, we have that $t_{k} \sigma^{\prime} \in\left\{t_{k} \sigma, n_{\epsilon}^{\epsilon}\right\}$ for any $k \in$ $\{1, \ldots, h\}$. Hence, we have that $e_{p}^{s i d_{p}} \neq \mathrm{Q}\left(t_{1} \sigma^{\prime}, \ldots, t_{h} \sigma^{\prime}\right)$, and thus $\left\langle\operatorname{exec}_{p}, T_{0}\right\rangle \models \psi \sigma^{\prime}$ (= $\left.\neg \mathrm{Q}\left(t_{1} \sigma^{\prime}, \ldots, t_{h} \sigma^{\prime}\right)\right)$.

- Case $\psi=$ learn $(t)$. In that case $t \sigma^{\prime} \in\left\{t \sigma, n_{\epsilon}^{\epsilon}\right\}$ (thanks to Condition 1 of Definition 4.3), then we know by hypothesis that $\mathrm{K}\left(\operatorname{exec}_{p}\right) \cup T_{0} \vdash t \sigma^{\prime}$ and thus, we conclude. 
- Case $\psi=\neg$ learn $(t)$. This case cannot occur because $\psi$ satisfies the conditions of an attack formula (see Definition 4.3), and in particular no learn $(u)$ appears negatively in $\psi$.

- Case $\psi=\mathrm{C}(t)$ or $\neg \mathrm{C}(t)$. In that case, we have that $t \sigma \in \mathcal{A}$. By construction, we have that $t \sigma=t \sigma^{\prime}$, and this allows us to conclude.

We now distinguish several inductive cases.

- Case $\psi=\psi_{1} \vee \psi_{2}$. Assume that $\left\langle\operatorname{exec}_{p}, T_{0}\right\rangle \models \psi_{1} \sigma$. The case where $\left\langle\operatorname{exec}_{p}, T_{0}\right\rangle \not \models \psi_{1} \sigma$ but $\left\langle\operatorname{exec}_{p}, T_{0}\right\rangle \models \psi_{2} \sigma$ can be proved in a similar way. By definition, we have that $\mathrm{Ws}\left(\operatorname{exec}_{p}, \psi_{1} \sigma\right)=\mathrm{Ws}\left(\operatorname{exec}_{p}, \psi \sigma\right) \subseteq S$. We can thus apply our inductive hypothesis to conclude that $\left\langle\operatorname{exec}_{p}, T_{0}\right\rangle \models \psi_{1} \sigma^{\prime}$ and thus $\left\langle\operatorname{exec}_{p}, T_{0}\right\rangle \models \psi \sigma^{\prime}\left(=\psi_{1} \sigma^{\prime} \vee \psi_{2} \sigma^{\prime}\right)$.

- Case $\psi=\neg\left(\psi_{1} \vee \psi_{2}\right)$. In that case, $\left\langle\operatorname{exec}_{p}, T_{0}\right\rangle \models \neg \psi_{1} \sigma$ and $\left\langle\operatorname{exec}_{p}, T_{0}\right\rangle \models \neg \psi_{2} \sigma$. By definition, we have that:

$$
\mathrm{Ws}\left(\operatorname{exec}_{p}, \neg \psi_{1} \sigma\right) \cup \mathrm{Ws}\left(\operatorname{exec}_{p}, \neg \psi_{2} \sigma\right)=\mathrm{Ws}\left(\operatorname{exec}_{p}, \psi \sigma\right) \subseteq S .
$$

By applying our inductive hypothesis, we obtain that $\left\langle\operatorname{exec}_{p}, T_{0}\right\rangle \models \neg \psi_{j} \sigma^{\prime}$ for $j \in\{1,2\}$. This allows us to conclude that $\left\langle\operatorname{exec}_{p}, T_{0}\right\rangle \models \psi \sigma^{\prime}\left(=\neg\left(\psi_{1} \sigma^{\prime} \vee \psi_{2} \sigma^{\prime}\right)\right)$.

- Case $\psi=\diamond \psi^{\prime}$. In that case, according to the semantics of our logic $\mathcal{L}$, there exists $j \leq i$ such that $\left\langle\operatorname{exec}_{j}, T_{0}\right\rangle \models \psi^{\prime} \sigma$, and thus by inductive hypothesis we know that $\left\langle\operatorname{exec}_{j}, T_{0}\right\rangle \models$ $\psi^{\prime} \sigma^{\prime}$. Hence, we have that $\left\langle\operatorname{exec}_{p}, T_{0}\right\rangle \models \psi \sigma^{\prime}\left(=\diamond \psi^{\prime} \sigma^{\prime}\right)$.

- Case $\psi=\neg \nabla \psi^{\prime}$. In that case, according to the semantics of our logic, we have that $\left\langle\operatorname{exec}_{p-1}, T_{0}\right\rangle \models \psi \sigma$ and $\left\langle\operatorname{exec}_{p}, T_{0}\right\rangle \models \neg \psi^{\prime} \sigma$. By inductive hypothesis we know that $\left\langle\operatorname{exec}_{p-1}, T_{0}\right\rangle \models \psi \sigma^{\prime}$. Note that, by definition of an attack formula (see Definition 4.3), there is no positive status event in $\psi \sigma$. Moreover, using our inductive hypothesis, we obtain that $\left\langle\operatorname{exec}_{p}, T_{0}\right\rangle \models \neg \psi^{\prime} \sigma^{\prime}$ (note that, again, by definition of an attack formula, we know that there is no positive status event in $\left.\neg \psi^{\prime} \sigma\right)$. This allows us to conclude that $\left\langle\operatorname{exec}_{p}, T_{0}\right\rangle \models \psi \sigma^{\prime}=\left(\neg \diamond \psi^{\prime} \sigma^{\prime}\right)$.

Lemma 7.6. Let $\Pi$ be a k-party protocol, and exec $=\left[\mathrm{e}_{1}^{\text {sid }}{ }_{1} ; \ldots ; \mathrm{e}_{\ell}^{\text {sid }}{ }_{\ell}\right]$ be a valid and wellformed execution of $\widetilde{\Pi}$ w.r.t. some set $T_{0}$ of ground atoms such that $T_{0} \cup \mathrm{K}(\mathrm{exec}) \forall k$ for any $k \in$ lgKeys $\backslash\left(\mathcal{K}_{\epsilon} \cup T_{0}\right)$. Let $\phi=\exists x_{1} \ldots . \exists x_{n} . \psi$ be an attack formula of $\mathcal{L}$, and $\sigma$ be a ground substitution such that $\left\langle\right.$ exec, $\left.T_{0}\right\rangle \models \psi \sigma$. Let $S$ be a set of session identifiers such that:

(1) Ws $($ exec, $\psi \sigma) \subseteq S$, and

(2) $\forall$ sess $_{1}$, sess $_{2}$ with ExpectedTag $($ exec, sess 1$)=$ ExpectedTag $\left(\right.$ exec, sess $\left.s_{2}\right)$, we have that sess $_{1} \in S$ if and only if sess $2 \in S$.

We have that exec $\left.\right|_{S}$ is an execution of $\widetilde{\Pi}$ that satisfies $\phi$, i.e. $\left\langle\left.\operatorname{exec}\right|_{S}, T_{0}\right\rangle \models \phi$.

Proof. First, we apply Lemma E.2 to ensure that the substitution $\sigma$ witnessing the fact that the attack formula $\phi$ is satisfiable only uses atomic terms and subterms that occur in St(exec, $S$ ). Hence, thanks to this lemma, we can assume w.l.o.g. that for all $j \in\{1, \ldots, n\}$, $\sigma\left(x_{j}\right) \in \mathrm{St}($ exec, $S) \cup \mathcal{A} \cup \operatorname{lgKeys} \cup \mathcal{N}_{\epsilon} \cup \mathcal{K}_{\epsilon}$. Then, we apply Lemma E.1 in order to conclude. 